\title{
WHITEHEAD TORSION
}

\section{BY J. MILNOR}

In 1935, Reidemeister, Franz and de Rham introduced the concept of "torsion" for certain finite simplicial complexes. For example let $X$ be a finite complex whose fundamental group $\pi_{1} X$ is cyclic of order $m$. We can identify $\pi_{1} X$ with the group of covering transformations of the universal covering complex $\hat{X}$. If $\pi_{1} X$ operates trivially on the rational homology $H_{*}(\hat{X} ; Q)$, then the torsion of $X$ is defined as a certain collection of elements in the algebraic number field $\boldsymbol{Q}[\exp (2 \pi i / m)]$. This torsion is a kind of determinant which describes the way in which the simplexes of $\hat{X}$ are fitted together with respect to the action of $\pi_{1} X$. The actual definition will be given in $\$ 8$. (See Franz [1935], de Rham [1950], Milnor [1961].)

In 1950, J. H. C. Whitehead defined the "torsion" of a homotopy equivalence between finite complexes. This is a direct generalization of the Reidemeister, Franz, and de Rham concept; but is a more delicate invariant. (See \$7.) It has gradually been realized that the Whitehead torsion provides a key tool for the study of combinatorial or differentiable manifolds with nontrivial fundamental group. Closely related is the concept of "simple homotopy type" (Whitehead [1939], [1950]).

The Whitehead torsion is not defined as an algebraic number, but rather as an element of a certain commutative group $\mathrm{Wh}\left(\pi_{1} X\right)$ which depends on the fundamental group. For many years these "Whitehead groups" were completely impossible to compute, except in a very few special cases (Higman [1940]). But recent work by H. Bass and others has made these groups moderately accessible.

The first six chapters of this presentation will be concerned with the algebraic part of the theory, and the remaining six chapters with the geometric applications. There are two appendices: one to show the relationship of Whitehead groups to the work of Bass and Mennicke on congruence subgroups, and one to help motivate the notation $K_{1} A$ which is used for the Whitehead group of a ring.

1. The Whitehead group $K_{1} A$ of a ring. Let $A$ be an associative ring with unit. The group of all nonsingular $n \times n$ matrices over $A$ will be denoted by $\mathrm{GL}(n, A)$. Identifying each $M \in \mathrm{GL}(n, A)$ with the matrix

$$
\left(\begin{array}{cc}
M & 0 \\
0 & 1
\end{array}\right) \in \mathrm{GL}(n+1, A)
$$


we obtain inclusions

$$
\mathrm{GL}(1, A) \subset \mathrm{GL}(2, A) \subset \mathrm{GL}(3, A) \subset \cdots
$$

The union is called the infinite general linear group $\mathrm{GL}(A)$.

A matrix is called elementary if it coincides with the identity matrix except for one off-diagonal element.

Lemma 1.1 (J. H. C. WhiteheAd). The subgroup $E(A) \subset \mathrm{GL}(A)$ generated by all elementary matrices is precisely equal to the commutator subgroup of $\mathrm{GL}(A)$.

Proof. Let $a E_{i j}$ denote the matrix with entry $a$ in the $(i, j)$ th place and zeros elsewhere. The identity

$$
\left(I+a E_{i j}\right)\left(I+E_{j k}\right)\left(I-a E_{i j}\right)\left(I-E_{j k}\right)=\left(I+a E_{i k}\right),
$$

for $i \neq j \neq k \neq i$, shows that each elementary matrix in $\operatorname{GL}(n, A)$ is a commutator, providing that $n \geqq 3$.

Conversely the following three identities show that each commutator $X Y X^{-1} Y^{-1}$ in $\mathrm{GL}(n, A)$ can be expressed as a product of elementary matrices within the larger group $\mathrm{GL}(2 n, A)$.

$$
\begin{aligned}
\left(\begin{array}{cc}
X Y X^{-1} Y^{-1} & 0 \\
0 & I
\end{array}\right) & =\left(\begin{array}{cc}
X & 0 \\
0 & X^{-1}
\end{array}\right)\left(\begin{array}{cc}
Y & 0 \\
0 & Y^{-1}
\end{array}\right)\left(\begin{array}{cc}
(Y X)^{-1} & 0 \\
0 & Y X
\end{array}\right), \\
\left(\begin{array}{cc}
X & 0 \\
0 & X^{-1}
\end{array}\right) & =\left(\begin{array}{cc}
I & X \\
0 & I
\end{array}\right)\left(\begin{array}{cc}
I & 0 \\
I-X^{-1} & I
\end{array}\right)\left(\begin{array}{cc}
I & -I \\
0 & I
\end{array}\right)\left(\begin{array}{cc}
I & 0 \\
I-X & I
\end{array}\right), \\
\left(\begin{array}{cc}
I & X \\
0 & I
\end{array}\right) & =\prod_{i=1}^{n} \prod_{j=n+1}^{2 n}\left(I+x_{i j} E_{i j}\right) .
\end{aligned}
$$

This completes the proof.

It follows that $E(A)$ is a normal subgroup of $\operatorname{GL}(A)$ with commutative quotient group. The quotient will be called the Whitehead group

$$
K_{1} A=\mathrm{GL}(A) / E(A) \text {. }
$$

We will usually think of $K_{1} A$ as an additive group.

Clearly $K_{1}$ is a covariant functor: that is, any ring homomorphism $A \rightarrow A^{\prime}$ gives rise to a group homomorphism $K_{1} A \rightarrow K_{1} A^{\prime}$.

If the ring $A$ happens to be commutative then we can also consider the special linear group $\mathrm{SL}(A)$, consisting of all matrices in $\mathrm{GL}(A)$ with determinant 1 . The quotient $\operatorname{SL}(A) / E(A)$ will be denoted by $S K_{1}(A)$, and called the special Whitehead group. Note the direct sum decomposition

$$
K_{1} A \cong U(A) \oplus S K_{1}(A)
$$


where $U(A)$ denotes the group of units in $A$. This follows immediately from the existence of the determinant homomorphism

$$
\operatorname{det}: \mathrm{GL}(A) / E(A) \rightarrow U(A),
$$

together with the inclusion

$$
U(A)=\mathrm{GL}(1, A) \subset \mathrm{GL}(A) .
$$

For many important rings the second summand $S K_{1}(A)$ is zero. In other words every matrix in $\operatorname{GL}(A)$ with determinant 1 can be reduced to the identity matrix by elementary row operations. ${ }^{1}$

Example 1.2. If $F$ is a field, then $S K_{1}(F)=0$. So the Whitehead group $K_{1} F$ can be identified with the group

$$
U(F)=F-\{0\}
$$

of units.

EXAMPLE 1.3. For the ring $\boldsymbol{Z}$ of rational integers, $S K_{1}(\boldsymbol{Z})=0$, so $K_{1} Z$ is equal to the group

$$
U(Z)=\{ \pm 1\}
$$

with two elements. More generally, if $A$ is any commutative ring which possesses a euclidean algorithm, then $S K_{1} A=0$.

The proofs of these statements are well known.

EXAMPLe 1.4. If $A$ has only finitely many maximal ideals, then $S K_{1} A=0$. (See Appendix 1; as well as Bass, Lazard and Serre [1964, Lemme 1].)

EXAMPLE 1.5. If $\mathcal{O}$ is the ring of integers in a finite extension field of the rational numbers, then $S K_{1} O=0$. (Bass and Milnor [to appear].)

More important for the applications is the following.

EXAMPLE 1.6. If $Z$ II is the integral group ring of a finite abelian group, then $S K_{1}(Z \Pi)=0$. This will be proved in a forthcoming paper by H. Bass. (Compare Appendix 1.)

Added in proof. Unfortunately, there seems to be serious difficulties with the proof of 1.6. If $\Pi$ is cyclic of prime order, the statement is certainly correct, but the general case remains in doubt. Care should be taken with $\S \S 6.4,6.7,8.1,12.8$, and other places in which 1.6 is used.

To break the monotony, here are some examples with $S K_{1} \neq 0$.

Example 1.7. Let $R[x, y]$ denote the Dedekind ring which is obtained from the real numbers $R$ by adjoining two indeterminates, subject to the single relation

${ }^{1}$ An elementary row operation on a matrix means the operation of left multiplying by an elementary matrix. 
Then the matrix

$$
x^{2}+y^{2}=1
$$

$$
\left(\begin{array}{rr}
x & y \\
-y & x
\end{array}\right) \in \operatorname{SL}(2, R[x, y])
$$

represents a nontrivial element of $S K_{1} R[x, y]$.

(See Appendix 2. More precisely it is possible to show that the group $S K_{1} R[x, y]$ is cyclic of order 2.)

ExAMPLE 1.8. Let $A=\boldsymbol{Z}\left(T \times T_{23}\right)$ be the integral group ring of the cartesian product of an infinite cyclic group $T$ and a cyclic group of order 23. Then $S K_{1} A \neq 0$.

This follows from Bass, Heller and Swan [1964], and will be discussed in Appendix 2. (In fact $S K_{1} A$ is the direct sum of a cyclic group of order 3 and an unknown 23-primary group.)

Finally, here are some examples in which the ring $A$ is noncommutative.

EXAMPLE 1.9. If $F$ is a skew-field, and $U=F^{*}$ the group of units, then $K_{1} F$ can be identified with the abelianized group $U /[U, U]$. The natural homomorphism

$$
\mathrm{GL}(n, F) \rightarrow K_{1} F
$$

is just the "noncommutative determinant" of Dieudonné [1943]. (See also Artin [1957].)

Lemma 1.10. Let $M_{n}(A)$ denote the ring of all $n \times n$ matrices over the ring $A$. Then

$$
K_{1} M_{n}(A) \cong K_{1} A
$$

Proof. Any $k \times k$ matrix with entries in $M_{n}(A)$ can be thought of as a $k n \times k n$ matrix with entries in $A$. In other words

$$
\mathrm{GL}\left(k, M_{n}(A)\right) \cong \mathrm{GL}(k n, A) .
$$

Passing to the limit as $k \rightarrow \infty$, and then abelianizing, we obtain the required isomorphism

$$
K_{1} M_{n}(A) \cong K_{1} A
$$

For one important class of rings the group $K_{1} A$ has been studied by Bass [1964]. We will say that $A$ is an order in a semisimple Q-algebra if the additive group of $A$ is a free abelian group of finite rank; and if the associated algebra $Q \otimes A$ over the rational numbers is semisimple. For example the integral group ring $Z \mathbb{Z}$ of any finite group $\Pi$ is an order in the semisimple algebra $Q \Pi$. 
Theorem 1.11 (BASS [1964]). If $A$ is an order in a semisimple Q-algebra then $K_{1} A$ is a finitely generated group of rank $r-q$, where $q$ denotes the number of simple factors of the algebra $Q \otimes A$, and $r$ denotes the number of simple factors in the corresponding real algebra $R \otimes A$.

As an example, let $A=Z T_{p}$ be the integral group ring of a cyclic group $T_{p}$ of prime order $p \geqq 3$. Then it is easy to verify that

$$
q=2, \quad r=\frac{1}{2}(p+1) ;
$$

so the Whitehead group

$$
K_{1}\left(Z T_{p}\right)=U\left(Z T_{p}\right)
$$

is a finitely generated abelian group of rank $(p-3) / 2$. (Compare Higman [1940].) In particular, this group has positive rank, providing that $p \geqq 5$.

We will pursue these ideas further in $\S 6$, and in the two appendices. The reader may prefer to skip immediately to $\S 6$.

2. Elementary constructions with free modules. The word "module" will always mean "finitely generated left $A$-module."

Following Whitehead, we impose the following mild restriction on the ring $A$ : The free module of rank $r$ over $A$ should not be isomorphic to the free module of rank $s$ if $r \neq s$. This restriction will always be satisfied, for example, if $A$ can be mapped homomorphically into a commutative ring.

Before proceeding it will be important to make a slight modification of the Whitehead groups studied in $\S 1$. Let $[-1] \in K_{1} A$ denote the element of order 2 corresponding to the unit

$$
(-1) \in \mathrm{GL}(1, A) \subset \mathrm{GL}(A) \text {. }
$$

Definition. The quotient $K_{1} A /\{0,[-1]\}$ will be denoted by $\bar{K}_{1} A$, and called the reduced ${ }^{2}$ Whitehead group of $A$.

Here are two important examples. For the integers $\boldsymbol{Z}$ the group $\bar{K}_{1} Z$ is zero. For the real numbers $R, \bar{K}_{1} R$ is isomorphic to the multiplicative group $R^{+}$of positive reals. A specific isomorphism is given by the correspondence $\left(a_{i j}\right) \rightarrow\left|\operatorname{det}\left(a_{i j}\right)\right|$.

The advantage of passing to this quotient is that two matrices which differ only by a permutation of the rows represent the same element of $\bar{K}_{1} A$. This follows, for example, from the fact that every permutation matrix represents the zero element of $\bar{K}_{1} Z \subset \bar{K}_{1} A$.

Let $F$ be a free $A$-module and let $b=\left(b_{1}, \cdots, b_{k}\right)$ and $c=\left(c_{1}, \cdots, c_{k}\right)$ be two different bases for $F$. Setting $c_{i}=\sum a_{i j} b_{j}$ we obtain a nonsingular matrix $\left(a_{i j}\right)$ with entries in $A$. The corresponding element of

${ }^{2}$ In some noncommutative cases it may happen that $[-1]=0$, so that $K_{1} A=\bar{K}_{1} A$. 
the reduced Whitehead group $\bar{K}_{1} A$ will be denoted by $[c / b]$. If $[c / b]=0$ then we will say that $b$ is equivalent to $c$ (briefly $b \sim c$ ). The identities

$$
\begin{aligned}
{[d / c]+[c / d] } & =[d / b], \\
{[b / b] } & =0
\end{aligned}
$$

show that this is an equivalence relation.

Examples. For a free module of rank 2 , the bases $\left(b_{1}+a b_{2}, b_{2}\right)$, $\left(b_{1}, a b_{1}+b_{2}\right)$, and $\left(b_{2}, b_{1}\right)$ are all equivalent to $\left(b_{1}, b_{2}\right)$.

Next consider a short exact sequence

$$
0 \rightarrow E \rightarrow F \rightarrow G \rightarrow 0
$$

of free modules. Given bases $e=\left(e_{1}, \cdots, e_{k}\right)$ for $E$ and $g=\left(g_{1}, \cdots, g_{l}\right)$ for $G$ we can construct a basis $e g$ for $F$ as follows. Lift each $g_{i} \in G$ to an element $g_{i}^{\prime}$ of $F$. Then

$$
e g=\left(e_{1}, \cdots, e_{k}, g_{i}^{\prime}, \cdots, g_{l}^{\prime}\right)
$$

is the required basis. Of course this basis $e g$ depends on the choice of the $g_{i}^{\prime}$. However the equivalence class of eg depends only on $e$ and $g$.

(Throughout $\$ 2$, the proofs are easy, and will be omitted.)

Note also the following identity. If $\bar{e}$ and $\bar{g}$ are alternative bases for the modules $E$ and $G$ respectively, then

$$
[\bar{e} \bar{g} / e g]=[\bar{e} / e]+[\bar{g} / g] .
$$

The following reformulation will of ten be convenient. Suppose that we are given free modules

$$
F_{0} \subset F_{1} \subset F_{2}
$$

together with bases $b_{1}$ for the free quotient module $F_{1} / F_{0}$ and $b_{2}$ for the quotient module $F_{2} / F_{1}$. Then we obtain a basis $b_{1} b_{2}$ for $F_{2} / F_{0}$, using the exact sequence

$$
0 \rightarrow F_{1} / F_{0} \rightarrow F_{2} / F_{0} \rightarrow F_{2} / F_{1} \rightarrow 0 .
$$

(2.2) This construction is associative: Given modules

$$
F_{0} \subset F_{1} \subset F_{2} \subset F_{3}
$$

and bases $b_{i}$ for the (free) quotient modules $F_{i} / F_{i-1}$, the basis $\left(b_{1} b_{2}\right) b_{3}$ for $F_{3} / F_{0}$ is equivalent to the basis $b_{1}\left(b_{2} b_{3}\right)$.

More generally, given modules

$$
F_{0} \subset F_{1} \subset \cdots \subset F_{k}
$$

and given bases $b_{i}$ for the quotients $F_{i} / F_{i-1}$, we obtain a basis $b_{1} b_{2} \cdots b_{k}$ for $F_{k} / F_{0}$ which is well defined up to equivalence. 
It will be convenient to represent situations such as this by lattice diagrams such as the following.

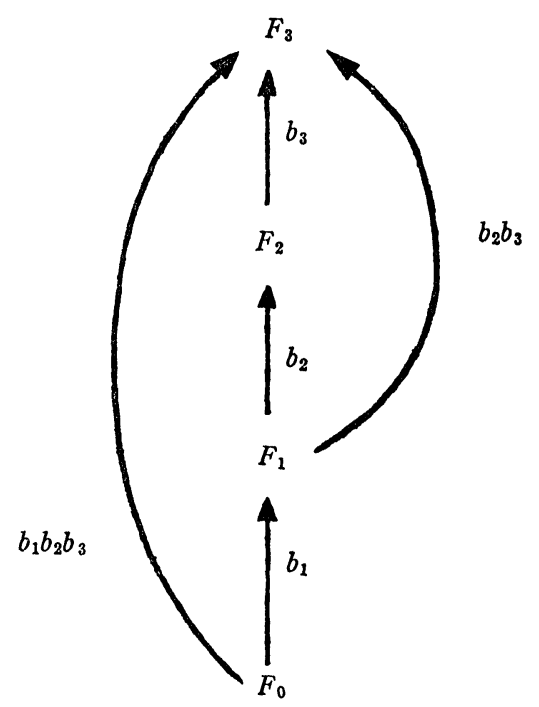

Each arrow represents an inclusion map. The letter associated with each arrow denotes a basis (or equivalence class of bases) for the corresponding quotient module.

Our construction is also commutative, in the following sense. Let $E$ and $F$ be submodules of $G$, and let $E+F$ denote the smallest submodule containing both. Thus we obtain a lattice:

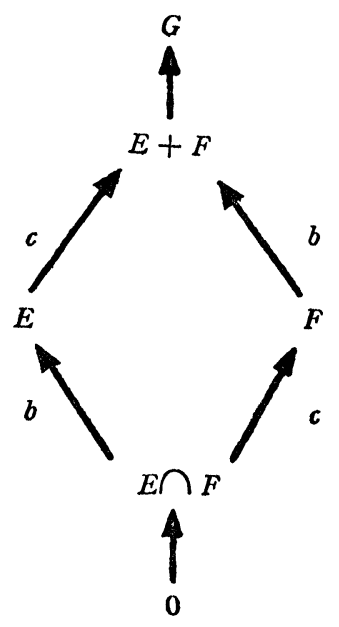


Suppose that $E / E \cap F \cong(E+F) / F$ is free with basis $b$ and that $F / E \cap F \cong(E+F) / E$ is free with basis $c$. Using the left hand inclusions we form the basis $b c$ for $(E+F) / E \cap F$, and using the right hand inclusions we form the basis $c b$. Then clearly

$$
b c \sim c b .
$$

It is essential at this point that we are working in $\bar{K}_{1} A$ rather than $K_{1} A$.

3. The torsion of a chain complex. Let $C_{n} \rightarrow C_{n-1} \rightarrow \cdots C_{1} \rightarrow C_{0}$ be a chain complex of modules over the ring $A$ such that each $C_{i}$ is free with a preferred basis $c_{i}$, and each homology group $H_{i}(C)$ is free with a preferred basis $h_{i}$. (The case $H_{i}(C)=0$ is of course not excluded. By definition, the zero module has a unique basis.) We wish to define the torsion $\tau(C) \in \bar{K}_{1} A$. Let $B_{i}$ denote the image of the boundary homomorphism $\partial: C_{i+1} \rightarrow C_{i}$ and let $Z_{i+1}$ denote its kernel. In order to simplify the discussion we will assume the following.

Hypothesis. Each $B_{i}$ is also a free module.

The more general situation in which this hypothesis is not satisfied will be put off until $\$$.

Choose a basis $b_{i}$ for each $B_{i}$. Using the inclusions $0 \subset B_{i} \subset Z_{i} \subset C_{i}$ where $Z_{i} / B_{i} \cong H_{i}, C_{i} / Z_{i} \cong B_{i-1}$ we see that the bases $b_{i}, h_{i}, b_{i-1}$ combine to yield a new basis $b_{i} h_{i} b_{i-1}$ for $C_{i}$. Now define $\tau(C)$ $=\sum(-1)^{i}\left[b_{i} h_{i} b_{i-1} / c_{i}\right]$. This does not depend on the choice of the $b_{i}$ since, choosing different bases $\bar{b}_{i}$, we have

$$
\sum(-1)^{i}\left[b_{i} h_{i} \bar{b}_{i-1} / c_{i}\right]=\sum(-1)^{i}\left(\left[b_{i} h_{i} b_{i-1} / c_{i}\right]+\left[\bar{b}_{i} / b_{i}\right]+\left[\bar{b}_{i-1} / b_{i-1}\right]\right),
$$

where the last two terms sum up to zero.

Of course $\tau(C)$ does depend on the $c_{i}$ and $h_{i}$.

Now consider a short exact sequence $0 \rightarrow C^{\prime} \rightarrow C \rightarrow C^{\prime \prime} \rightarrow 0$, in the category of chain complexes and chain mappings over $A$. We will assume that the modules $C_{i}^{\prime}, C_{i}, C_{i}^{\prime \prime}$ are free with preferred bases $c_{i}^{\prime}, c_{i}, c_{i}^{\prime \prime}$ which are compatible, in the sense that $c_{i} \sim c_{i}^{\prime} c_{i}^{\prime \prime}$.

We wish to prove that $\tau(C)$ is equal to $\tau\left(C^{\prime}\right)+\tau\left(C^{\prime \prime}\right)$ plus a correction term which depends on the homology groups of the three chain complexes. First consider the special case where all three complexes are acyclic.

THEOREM 3.1. If the homology modules $H_{*} C^{\prime}, H_{*} C$ and $H_{*} C^{\prime \prime}$ are all zero, then $\tau(C)=\tau\left(C^{\prime}\right)+\tau\left(C^{\prime \prime}\right)$. 
The proof will be based on the following lattice of submodules of $C_{i}$.

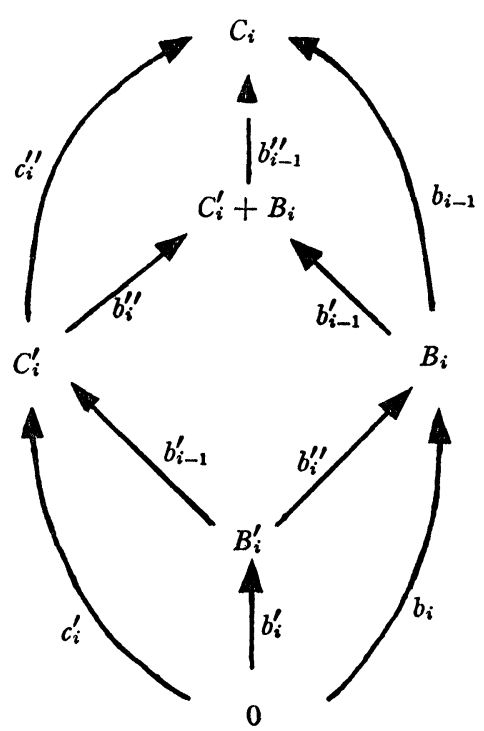

Each capital letter in the diagram represents a submodule of $C_{i}$. Note that $C_{i}^{\prime} \cap B_{i}=B_{i}^{\prime}$. The natural isomorphisms

$$
\begin{aligned}
C_{i} /\left(C_{i}^{\prime}+B_{i}\right) & \cong C_{i}^{\prime \prime} / B_{i}^{\prime \prime} \cong B_{i-1}^{\prime \prime}, \\
C_{i}^{\prime} / B_{i}^{\prime} & \cong B_{i-1}^{\prime}
\end{aligned}
$$

and

$$
B_{i} / B_{i}^{\prime} \cong\left(C_{i}^{\prime}+B_{i}\right) / C_{i}^{\prime} \cong B_{i}^{\prime \prime}
$$

are easily verified.

Each lower case letter in the diagram represents a basis for the corresponding quotient module. We are assuming that the modules $B_{i}^{\prime}, B_{i}, B_{i}^{\prime \prime}$ are free. Let $b_{i}^{\prime}, b_{i}, b_{i}^{\prime \prime}$ be bases for these modules. Since we can choose any bases we like, we may as well choose $b_{i}$ to be equal to $b_{i}^{\prime} b_{i}^{\prime \prime}$. Hence, pushing to the left across the diagram, we have

$$
b_{i} b_{i-1}=b_{i}^{\prime}\left(b_{i}^{\prime \prime} b_{i-1}^{\prime}\right) b_{i-1}^{\prime \prime} \sim\left(b_{i}^{\prime} b_{i-1}^{\prime}\right)\left(b_{i}^{\prime \prime} b_{i-1}^{\prime}\right),
$$

using $\S 2.3$. Therefore

$$
\begin{aligned}
\tau(C) & =\sum(-1)^{i}\left[b_{i} b_{i-1} / c_{i}\right] \\
& =\sum(-1)^{i}\left[\left(b_{i}^{\prime} b_{i-1}^{\prime}\right)\left(b_{i}^{\prime \prime} b_{i-1}^{\prime \prime}\right) / c_{i}^{\prime} c_{i}^{\prime \prime}\right] \\
& =\sum(-1)^{i}\left[\left(b_{i}^{\prime} b_{i-1}^{\prime} / c_{i}^{\prime}\right]+\left[b_{i}^{\prime \prime} b_{i-1}^{\prime} / c_{i}^{\prime \prime}\right]\right) \\
& =\tau\left(C^{\prime}\right)+\tau\left(C^{\prime \prime}\right) ;
\end{aligned}
$$


using \$2.1. This completes the proof of Theorem 3.1.

More generally suppose that the homology groups of $C^{\prime}, C$, and $C^{\prime \prime}$ are not zero; but are free with preferred bases. Then the exact homology sequence

$$
H_{n}^{\prime} \rightarrow H_{n} \rightarrow H_{n}^{\prime \prime} \rightarrow H_{n-1}^{\prime} \rightarrow \cdots \rightarrow H_{0}^{\prime} \rightarrow H_{0} \rightarrow H_{0}^{\prime \prime}
$$

can be thought of as a free acyclic chain complex ${ }^{3}$ te of dimension $3 n+2$. Hence the torsion $\tau$ HC is defined.

ThEOREM 3.2. With these assumptions the torsion $\tau(C)$ is equal to $\tau\left(C^{\prime}\right)+\tau\left(C^{\prime \prime}\right)+\tau(\mathcal{H C})$.

Proof. Let $X_{i}^{\prime} \subset H_{i}^{\prime}$ and $X_{i} \subset H_{i}$ be the kernels of the homomorphisms $H_{i}^{\prime} \rightarrow H_{i}$ and $H_{i} \rightarrow H_{i}^{\prime \prime}$ respectively. Similarly let $X_{i}^{\prime \prime}$ be the kernel of $H_{i}^{\prime \prime} \rightarrow H_{i-1}^{\prime}$. Thus the sequences

$$
\begin{aligned}
& 0 \rightarrow X_{i}^{\prime} \rightarrow H_{i}^{\prime} \rightarrow X_{i} \rightarrow 0 \\
& 0 \rightarrow X_{i} \rightarrow H_{i} \rightarrow X_{i}^{\prime \prime} \rightarrow 0 \\
& 0 \rightarrow X_{i}^{\prime \prime} \rightarrow H_{i}^{\prime \prime} \rightarrow X_{i-1}^{\prime} \rightarrow 0
\end{aligned}
$$

are exact. We are assuming that the modules $X_{i}^{\prime}, X_{i}$ and $X_{i}^{\prime \prime}$ are free. Choose bases $x_{i}^{\prime}, x_{i}$ and $x_{i}^{\prime \prime}$ respectively.

Consider the following lattice of submodules of $C_{i}$.

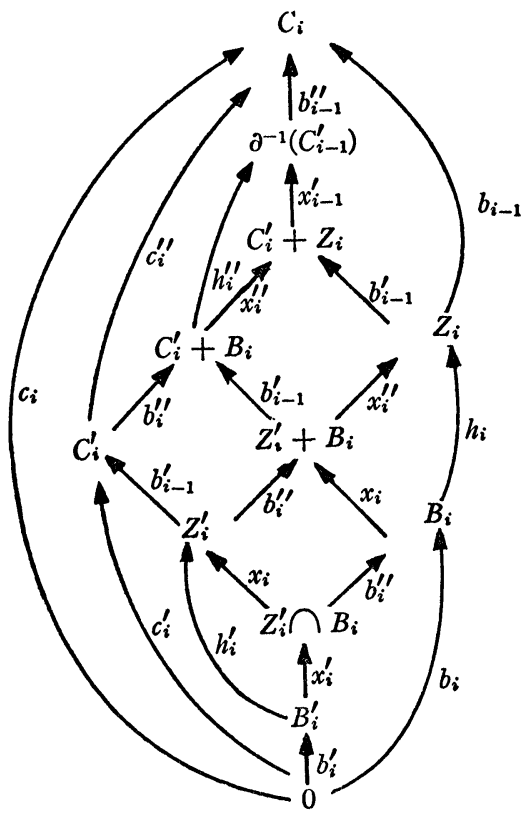

${ }^{3}$ To be more precise, we define $\mathcal{H C}$ by setting $\mathcal{H} \mathcal{C}_{3 i}=H_{i}^{\prime \prime}, \mathcal{F C}_{3 i+1}=H_{i}, \mathfrak{H C}_{3 i+2}=H_{i}^{\prime}$. 
The notation is mostly self explanatory. For example $\partial^{-1}\left(C_{i-1}^{\prime}\right)$ denotes the set of all $\gamma \in C_{i}$ such that $\partial(\gamma)$ belongs to $C_{i-1}^{\prime}$ (and hence to $C_{i-1}^{\prime} \cap B_{i-1}=Z_{i-1}^{\prime} \cap B_{i-1}$ ). The verification that this diagram exists as advertised will be left to the reader.

Again we have $c_{i} \sim c_{i}^{\prime} c_{i}^{\prime \prime}$ by hypothesis. We are free to choose the bases $b_{i}^{\prime}, b_{i}, b_{i}^{\prime \prime}$, so we may choose $b_{i}$ equal to $b_{i}^{\prime} x_{i}^{\prime} b_{i}^{\prime \prime}$. By definition

$$
\begin{aligned}
\tau(\mathcal{F C})= & \sum(-1)^{3 i+2}\left[x_{i}^{\prime} x_{i} / h_{i}^{\prime}\right] \\
& +\sum(-1)^{3 i+1}\left[x_{i} x_{i}^{\prime \prime} / h_{i}\right] \\
& +\sum(-1)^{3 i}\left[x_{i}^{\prime \prime} x_{i-1}^{\prime} / h_{i}^{\prime \prime}\right] .
\end{aligned}
$$

Hence

$$
\begin{array}{ccc}
\tau\left(C^{\prime}\right) & -\tau(C) & +\tau\left(C^{\prime \prime}\right)+\tau(\text { JC }) \\
=\sum(-1)^{i}\left(\left[b_{i}^{\prime} h_{i}^{\prime} b_{i-1}^{\prime} / c_{i}^{\prime}\right]\right. & -\left[b_{i} h_{i} b_{i-1} / c_{i}\right]+\left[b_{i}^{\prime \prime} h_{i}^{\prime \prime} b_{i}^{\prime \prime}-1 / c_{i}^{\prime \prime}\right] \\
\left.+\left[x_{i}^{\prime} x_{i} / h_{i}^{\prime}\right]-\left[x_{i} x_{i}^{\prime \prime} / h_{i}\right]+\left[x_{i}^{\prime \prime} x_{i-1}^{\prime} / h_{i}^{\prime \prime}\right]\right) .
\end{array}
$$

We must prove that this expression is zero. But, upon inspecting the expression, we see that it does not depend on the choice of $h_{i}^{\prime}, h_{i}$ and $h_{i}^{\prime \prime}$. Hence we may as well assume that

$$
h_{i}^{\prime}=x_{i}^{\prime} x_{i}, \quad h_{i}=x_{i} x_{i}^{\prime \prime}, \quad h_{i}^{\prime \prime}=x_{i}^{\prime \prime} x_{i-1}^{\prime}
$$

so that $\tau(\mathfrak{K C})=0$.

Similarly, after substituting $c_{i}^{\prime} c_{i}^{\prime \prime}$ for $c_{i}$, the expression does not depend on the choice of $c_{i}^{\prime}$ and $c_{i}^{\prime \prime}$. Hence we may as well assume that

$$
c_{i}^{\prime}=b_{i}^{\prime} h_{i}^{\prime} b_{i-1}^{\prime}, \quad c_{i}^{\prime \prime}=b_{i}^{\prime \prime} h_{i}^{\prime \prime} b_{i-1}^{\prime \prime}
$$

so that $\tau\left(C^{\prime}\right)=\tau\left(C^{\prime \prime}\right)=0$.

Making these assumptions, it only remains to verify that $\tau(C)=0$. Pushing to the left across our diagram, we have the following equivalences.

$$
\begin{aligned}
b_{i} h_{i} b_{i-1} & =b_{i}^{\prime} x_{i}^{\prime}\left(b_{i}^{\prime \prime} x_{i}\right)\left(x_{i}^{\prime \prime} b_{i-1}^{\prime}\right) x_{i-1}^{\prime} b_{i-1}^{\prime \prime} \\
& \sim b_{i}^{\prime} x_{i}^{\prime} x_{i}\left(b_{i}^{\prime \prime} b_{i-1}^{\prime}\right) x_{i}^{\prime \prime} x_{i-1}^{\prime} b_{i-1}^{\prime \prime} \\
& \sim b_{i}^{\prime}\left(x_{i}^{\prime} x_{i}\right) b_{i-1}^{\prime} b_{i}^{\prime \prime}\left(x_{i}^{\prime \prime} x_{i-1}^{\prime}\right) b_{i-1}^{\prime \prime} \\
& =\left(b_{i}^{\prime} h_{i}^{\prime} b_{i-1}^{\prime}\right)\left(b_{i}^{\prime \prime} h_{i}^{\prime \prime} b_{i-1}^{\prime \prime}\right) \\
& =c_{i}^{\prime} c_{i}^{\prime \prime} \sim c_{i} .
\end{aligned}
$$

Therefore

$$
\tau(C)=\sum(-1)^{i}\left[b_{i} h_{i} b_{i-1} / c_{i}\right]
$$

is equal to zero. This completes the proof. 
4. Stably free modules. A module $M$ over $A$ will be called stably free if the direct sum of $M$ with some free module is free. (Recall that all modules are to be finitely generated.) If $M \oplus F \cong F^{\prime}$ then the difference between the rank of $F^{\prime}$ and the rank of $F$ will be called the rank $r$ of $M$.

Lemma 4.1. Consider a short exact sequence $0 \rightarrow X \rightarrow Y \rightarrow Z \rightarrow 0$ of $A$-modules. If $Y$ and $Z$ are stably free, then $X$ is also stably free.

Proof. Since $Z$ is projective the sequence splits; so that $Y$ is isomorphic to $X \oplus Z$. Thus if $Z \oplus F \cong F^{\prime}$ and $Y \oplus F \cong F^{\prime \prime}$, where $F, F^{\prime}$, $F^{\prime \prime}$ are free, it follows that $X \oplus F^{\prime} \cong F^{\prime \prime}$.

Now consider a free chain complex

$$
C_{n} \rightarrow C_{n-1} \rightarrow \cdots \rightarrow C_{0}
$$

over $A$, and assume that the homology modules $H_{i}=H_{i}(C)$ are also free. Using the exact sequences

$$
\begin{aligned}
& 0 \rightarrow Z_{i} \rightarrow C_{i} \rightarrow B_{i-1} \rightarrow 0 \\
& 0 \rightarrow B_{i} \rightarrow Z_{i} \rightarrow H_{i} \longrightarrow 0
\end{aligned}
$$

it follows by induction that all of the modules $Z_{i}$ and $B_{i}$ are stably free.

We will show that the constructions of $\$ 2$ can be carried out using stably free modules in place of free modules. It will then follow easily that all of the constructions and proofs of $\$ 3$ can be carried out without the extra hypothesis that the $B_{i}\left(\right.$ and $\left.X_{i}\right)$ are free.

Let $F_{i}$ denote a standard free module of rank $i$, with standard basis $f_{1}, \cdots, f_{i}$. We may think of $F_{i}$ as the submodule of $F_{i+1}$ generated by the first $i$ basis elements.

Definition. An s-basis $b$ for a stably free module $M$ is a basis $\left(b_{1}, \cdots, b_{r+t}\right)$ for some direct sum $M \oplus F_{t}$. Here $t$ can be any nonnegative integer.

Given two s-bases $b=\left(b_{1}, \cdots, b_{r+t}\right)$ and $c=\left(c_{1}, \cdots, c_{r+u}\right)$ for $M$, the symbol

$$
[c / b] \in \bar{K}_{1} A
$$

is defined as follows. Choose an integer $v \geqq \operatorname{Max}(t, u)$. Extend $\left(b_{1}, \cdots, b_{r+t}\right)$ to a basis for $M \oplus F_{v}$ by setting

$$
b_{r+i}=0 \oplus f_{i}, \quad i \geqq t+1 .
$$

Similarly extend $\left(c_{1}, \cdots, c_{r+u}\right)$ to a basis for $M \oplus F_{v}$ by setting

$$
c_{r+i}=0 \oplus f_{i}, \quad i \geqq u+1 .
$$

Now $c_{i}=\sum a_{i j} b_{j}$ where the matrix $\left(a_{i j}\right) \in \mathrm{GL}(r+v, A)$ represents the 
required element $[c / b] \in \bar{K}_{1} A$. Clearly this construction does not depend on the choice of $v$. As in $\S 2$, we write $b \sim c$ if $[c / b]=0$. The identities

$$
\begin{aligned}
& {[d / b]=[d / c]+[c / b]} \\
& {[b / b]=0}
\end{aligned}
$$

are again satisfied.

Next consider a short exact sequence

$$
0 \rightarrow X \rightarrow Y \rightarrow Z \rightarrow 0 \text {. }
$$

Given s-bases $x$ for $X$ and $z$ for $Z$ we can construct an s-basis $x z$ for $Y$ as follows. Suppose that $x$ is a basis for $X \oplus F_{t}$ and that $z$ is a basis for $Z \oplus F_{u}$. Consider the exact sequence

$$
0 \rightarrow F_{t} \stackrel{\alpha}{\rightarrow} F_{t+u} \stackrel{\beta}{\rightarrow} F_{u} \rightarrow 0
$$

where $\alpha$ denotes the inclusion homomorphism, and

$$
\beta\left(f_{i}\right)= \begin{cases}0 & \text { for } i \leqq t \\ f_{i-t} & \text { for } i>t .\end{cases}
$$

Forming the direct sum

$$
0 \oplus 0 \rightarrow X \oplus F_{t} \rightarrow Y \oplus F_{t+u} \rightarrow Z \oplus F_{u} \rightarrow 0 \oplus 0,
$$

the bases $x$ and $z$ combine to yield a basis $x z$ for $Y \oplus F_{t+u}$, which is well defined up to equivalence. This is the required construction.

Lemma 4.2. This composition operation for s-basis is "associative" and "commutative" in the sense of $\$ 2$. Furthermore, if $\bar{x}$ and $\bar{z}$ are alternative s-bases for $X$ and $Z$ respectively, then

$$
[\bar{x} \bar{z} / x z]=[\bar{x} / x]+[\bar{z} / z]
$$

just as in $\$ 2$.

The proof, which is rather dull, will be omitted.

It is now possible to revise $\$ 3$ by substituting "stably free" and "s-basis" wherever the words "free" and "basis" occur. With these changes, all of the arguments still go through; but no extra hypothesis is needed to guarantee that the modules $B_{i}$ and $X_{i}$ are stably free.

5. An algebraic "subdivision" theorem. This section will prove an algebraic theorem which will enable us later to handle the geometric operations of subdividing a complex, or triangulating a smooth manifold. 
The following situation is frequently encountered in homology theory. Let $C$ be a chain complex and let

$$
C^{(0)} \subset C^{(1)} \subset \cdots \subset C^{(n)}=C
$$

be a "filtration" of $C$ by subcomplexes such that the following is satisfied. We set $C^{(-1)}=0$.

Hypothesis. The homology group $H_{i}\left(C^{(\lambda)} / C^{(\lambda-1)}\right)$ is zero for $i \neq \lambda$.

Then we can define a new chain complex $\bar{C}$ by setting

$$
\bar{C}_{\lambda}=H_{\lambda}\left(C^{(\lambda)} / C^{(\lambda-1)}\right) \text {. }
$$

The boundary homomorphism $\partial: \bar{C}_{\lambda} \rightarrow \bar{C}_{\lambda-1}$ is to be obtained from the exact sequence of the triple $C^{(\lambda)}, C^{(\lambda-1)}, C^{(\lambda-2)}$. The following is well known.

Lemma 5.1. The homology groups $H_{i} \bar{C}$ are canonically isomorphic to the groups $H_{i} C$.

For completeness, here is a proof. The fact that

$$
H_{i} C^{(\lambda)} / C^{(\mu)}=0 \text { for } i>\lambda \text { or } i \leqq \mu
$$

is proved by an easy induction on $\lambda-\mu$. Hence

$$
H_{i} C \cong H_{i} C^{(i+1)} \cong H_{i}\left(C^{(i+1)} / C^{(i-2)}\right) .
$$

Now consider the diagram

$$
\bar{C}_{\lambda+1} \rightarrow H_{\lambda} C_{C_{\lambda-1}^{(\lambda)}}^{0} C^{(\lambda-2)} \rightarrow H_{\lambda} C^{(\lambda+1)} / C^{(\lambda-2)} \rightarrow 0
$$

where the vertical line comes from the homology exact sequence of the triple $C^{(\lambda)}, C^{(\lambda-1)}, C^{(\lambda-2)}$; and the horizontal line from the triple $C^{(\lambda+1)}, C^{(\lambda)}, C^{(\lambda-2)}$. Inspection shows that the cycle group $\bar{Z}_{\lambda}$ of $\bar{C}$ can be identified with $H_{\lambda} C^{(\lambda)} / C^{(\lambda-2)}$. Hence

$$
H_{\lambda} \bar{C}=\bar{Z}_{\lambda} / \bar{B}_{\lambda} \cong H_{\lambda} C^{(\lambda+1)} / C^{(\lambda-2)}=H_{\lambda} C,
$$

which completes the proof.

We wish to examine the behavior of the torsion in a filtered chain complex of this type. 
Further Hypotheses. Each $C_{i}^{(\lambda)} / C_{i}^{(\lambda-1)}$ should be free with preferred basis $c_{i}^{\lambda}$; so that $C_{i}$ is free with basis

$$
c_{i}=\stackrel{0}{0} c_{i} c_{i} c_{i}^{2} \cdots c_{i}^{n} .
$$

Also each $\bar{C}_{\lambda}=H_{\lambda} C^{(\lambda)} / C^{(\lambda-1)}$ should be free with preferred basis $\bar{c}_{\lambda}$; and the modules

$$
H_{i} C \cong H_{i} \bar{C}
$$

should be free with preferred basis $h_{i}$. Finally $C$ should be finitely generated so that the torsions $\tau C$ and $\tau \bar{C}$ are defined.

With these hypotheses we will prove:

AlgeBraic subdivision THEOREM 5.2. If each quotient complex $C^{(k)} / C^{(k-1)}$ has torsion $\tau\left(C^{(k)} / C^{(k-1)}\right)$ equal to zero, then $\tau(C)=\tau(\bar{C})$.

Proof. Let $\bar{C}^{(k)}$ denote the truncated chain complex

$$
\cdots 0 \rightarrow \bar{C}_{k} \rightarrow \bar{C}_{k-1} \rightarrow \cdots \rightarrow \bar{C}_{0} \rightarrow 0
$$

which is obtained from $\bar{C}$ by chopping off the chain modules of degree $>k$. We wish to look at the torsion $\tau\left(\bar{C}^{(k)}\right)$. It follows from 5.1 that $H_{i} \bar{C}^{(k)} \cong H_{2} C^{(k)}$. Note that $H_{i} \bar{C}^{(k)} \cong H_{i} C^{(k)}$ is zero for $i>k$; and is isomorphic to $H_{i} C$, with preferred basis $h_{i}$, for $i<k$. In order for the torsion to be defined we must also choose a preferred basis (or at least s-basis) for $H_{k} \bar{C}^{(k)} \cong H_{k} C^{(k)}$. But clearly $H_{k} \bar{C}^{(k)}$ can be identified with the cycle group $\bar{Z}_{k}$ of $\bar{C}$, which is known to be stably free. Hence some preferred s-basis $\bar{z}_{k}$ can be chosen. It then follows that $\tau\left(\bar{C}^{(k)}\right)$ and $\tau\left(C^{(k)}\right)$ are defined.

We will prove by induction on $k$ that

$$
\tau\left(C^{(k)}\right)=\tau\left(\bar{C}^{(k)}\right) .
$$

Taking $k=n$, this will complete the proof.

Let $\mathcal{H}$ denote the homology exact sequence of the pair $\left(\bar{C}^{(k)}\right.$, $\left.\bar{C}^{(k-1)}\right)$; or equivalently of the pair $\left(C^{(k)}, C^{(k-1)}\right)$. Then according to $\$ 3$ we have

$$
\begin{aligned}
& \tau \bar{C}^{(k)}=\tau \bar{C}^{(k-1)}+\tau \bar{C}^{(k)} / \bar{C}^{(k-1)}+\tau \mathcal{H C}, \\
& \tau C^{(k)}=\tau C^{(k-1)}+\tau C^{(k)} / C^{(k-1)}+\tau \mathcal{H C} .
\end{aligned}
$$

But $\tau \bar{C}^{(k-1)}=\tau C^{(k-1)}$ by induction, and $\tau C^{(k)} / C^{(k-1)}=0$ by hypothesis. Since $\tau \bar{C}^{(k)} / \bar{C}^{(k-1)}$ is trivially zero, it follows that $\tau \bar{C}^{(k)}$ $=\tau C^{(k)}$. This completes the proof.

6. The Whitehead group $\mathrm{Wh}(I I)$ of a group. Let II denote a multiplicative group and $Z \Pi$ the corresponding integral group ring. Then clearly $I$ itself is contained in the group of units 


$$
U(Z \Pi)=\mathrm{GL}(1, Z \Pi) \subset \mathrm{GL}(Z \Pi) .
$$

Hence there are natural homomorphisms ${ }^{4}$

$$
\Pi \rightarrow K_{1}(Z \Pi) \rightarrow \bar{K}_{1}(Z \Pi) .
$$

Definition. The cokernel

$$
\bar{K}_{1}(Z \Pi) / \text { image (I) }
$$

is called the Whitehead group $\mathrm{Wh}(\Pi)$. This construction can be described by the exact sequence

$$
0 \rightarrow \mathrm{II} /[\Pi, \Pi] \rightarrow \bar{K}_{1}(Z \Pi) \rightarrow \mathrm{Wh}(\Pi) \rightarrow 0 .
$$

Clearly $\mathrm{Wh}(\Pi)$ is a covariant functor of $\Pi$. In other words, any homomorphism $f: \Pi_{1} \rightarrow \Pi_{2}$ induces a homomorphism $f_{*}:$ Wh $\left(\Pi_{1}\right)$ $\rightarrow \mathrm{Wh}\left(\Pi_{2}\right)$.

Lemma 6.1. If $f: \Pi \rightarrow \Pi$ is an inner automorphism, then $f_{*}: \mathrm{Wh}(\Pi)$ $\rightarrow \mathrm{Wh}(\mathrm{II})$ is the identity.

Proof. Let $f(\sigma)=\phi \sigma \phi^{-1}$ for each $\sigma \in \Pi$. The corresponding automorphism of $\mathrm{GL}(n, Z \Pi)$ is clearly given by

$$
\left(a_{i j}\right) \mapsto\left(^{\phi} \cdot{ } \cdot{ }_{\phi}\left(a_{i j}\right){ }^{\phi} \cdot{ }^{\phi} \cdot{ }^{-1} \cdot\right.
$$

Passing to the abelian group $K_{1} Z \Pi$, or to $W h(\Pi)$, we therefore obtain the identity automorphism.

Definitions. If $a=\sum n_{i} \sigma_{i}$ is an element of $Z$ III (with $n_{i} \in \boldsymbol{Z}$, $\left.\sigma_{i} \in \Pi\right)$ then the element $\sum n_{i} \sigma_{i}^{-1}$ is called the conjugate of $a$, and will be denoted briefly by $\bar{a}$. This conjugation operation is an antiautomorphism of the group ring. That is the conjugate of $a+b$ is $\bar{a}+\bar{b}$ and

$$
\overline{a b}=\bar{b} \bar{a} .
$$

A corresponding anti-automorphism of the general linear group $\mathrm{GL}(Z \mathrm{II})$ is obtained by sending each matrix $\left(a_{i j}\right)$ into its conjugate transpose $\left(\bar{a}_{j i}\right)$. Passing to the abelianized group $K_{1} Z \Pi$ this gives rise to an automorphism. Hence one obtains an automorphism of Wh(i)). The image of an element $\omega \in \mathrm{Wh}(I I)$ will be denoted by the symbol $\bar{\omega}$, and called the conjugate of $\omega$.

Miscellaneous quoted theorems. If $\Pi$ is infinite cyclic, or is finite of order 2, 3 or 4 then Higman [1940] proved that Wh(II) is zero. Bass' Heller and Swan [1964] have shown that the Whitehead group of any free abelian group is zero. Stallings [1965 $]$ and Gersten [1965 ] have

\footnotetext{
${ }^{4}$ Here $\bar{K}_{1} A=K_{1} A /\{0,[-1]\}$, as in $\S 2$.
} 
shown that the Whitehead group of any free group is zero. In fact the Whitehead group of a free product is given by

$$
\mathrm{Wh}\left(\Pi * \Pi^{\prime}\right)=\mathrm{Wh}(\Pi) \oplus \mathrm{Wh}\left(\Pi^{\prime}\right) .
$$

(There is no corresponding formula for the Whitehead group of a cartesian product. For example $\mathrm{Wh}\left(T_{3}\right)=0$ and $\mathrm{Wh}\left(T_{4}\right)=0$, but $\mathrm{Wh}\left(T_{3} \times T_{4}\right) \cong \boldsymbol{Z}$; where $T_{m}$ denotes the cyclic group of order $m$.)

If $I I$ is finite then Bass [1964] has shown that Wh(II) is finitely generated. In fact, more precisely:

TheOREM 6.2 (BAss). If $\Pi$ is finite then $\mathrm{Wh}(\Pi)$ is a finitely generated abelian group of rank $r-q$, where $r$ is the number of irreducible real representations of $\Pi$, and $q$ is the number of irreducible rational representations.

(This is a special case of $\$ 1.11$.$) These numbers can be described$ quite explicitly as follows: $q$ is the number of conjugate classes of cyclic subgroups ${ }^{5}$ of $\Pi$, and $r$ is the number of conjugate classes of unordered pairs $\left\{\sigma, \sigma^{-1}\right\}$. Hence for $\Pi$ finite we have:

Corollary 6.3. The Whitehead group $\mathrm{Wh}(\mathrm{II})$ of a finite group is itself finite if and only if, for every two elements $\sigma, \tau \in \Pi$ which generate the same cyclic subgroup, either $\sigma$ is conjugate to $\tau$ or $\sigma^{-1}$ is conjugate to $\tau$.

As an example, the symmetric group $S_{n}$ consisting of all permutations of $n$ elements satisfies this condition. (In fact it is true that every complex representation of $S_{n}$ is actually rational.) Hence $\mathrm{Wh}\left(S_{n}\right)$ is finite. I do not know whether or not $\mathrm{Wh}\left(S_{n}\right)$ is zero.

Now suppose that $\Pi$ is finite abelian. Then, according to $\$ 1.6$ the group $K_{1} Z \Pi$ is isomorphic to the group $U \subset Z I I$ of units in $Z I I$. (See also Appendix 1.) But according to Higman the only elements of finite order in $U$ are the group elements and their negatives. Hence:

Theorem 6.4 (BAss). If $\Pi$ is finite abelian then $\mathrm{Wh}(\Pi)$ is a free abelian group of rank $r-q$.

Corollary 6.5. The Whitehead group $\mathrm{Wh}(\mathrm{II})$ of a finite abelian group $\Pi$ is zero if and only if the group $\Pi$ has exponent 1, 2, 3, 4, or 6.

For the cyclic groups of order 1, 2, 3, 4 and 6 are the only ones which have the property that any two generators are equal up to sign.

Here is a simple example worked out in more detail.

EXAMPLE 6.6. Let $T_{5}$ be cyclic of order 5 with generator $t$. Then $\mathrm{Wh}\left(T_{5}\right)$ is infinite cyclic; and the unit

\footnotetext{
- In the abelian case $q=\sum \operatorname{order}\left(\Pi \otimes \boldsymbol{Z}_{m}\right) / m$, summed over all $m$ dividing order(II).
} 


$$
u=t+t^{-1}-1 \in Z T_{5}
$$

represents a generator. ${ }^{6}$

(In fact the identity

$$
\left(t+t^{-1}-1\right)\left(t^{2}+t^{-2}-1\right)=1
$$

shows that $u$ is a unit; and the homomorphism

$$
\begin{aligned}
t & \mapsto \exp (2 \pi i / 5)=\xi, \\
u & \mapsto \xi+\xi^{-1}-1=2 \cos 72^{\circ}-1
\end{aligned}
$$

from $\boldsymbol{Z} T_{5}$ to the complex numbers can be used to show that no power of $u$ is equal to 1 . The rest of the argument is much more difficult.)

REMARK. This unit $u \in Z T_{5}$ is clearly self-conjugate. It is interesting to note that the automorphism $t \rightarrow t^{2}$ of $T_{5}$ carries $u$ to $u^{-1}$. If we embed $T_{5}$ in the group $G$ of order 20 in which $t$ is conjugate to $t^{2}$, this implies that the unit $u$ represents an element of order $\leqq 2$ in the finite group $\mathrm{Wh}(G)$. I do not know whether or not $\mathrm{Wh}(G)$ is zero.

Here is a more general statement.

Lemмa 6.7. If $\Pi$ is finite abelian, then every element of $\mathrm{Wh}(\Pi)$ is self-conjugate.

Proof. If $u$ is a unit of $Z \Pi$, it is sufficient to show that $\bar{u} / u$ is an element of finite order in the group of units. The rational group ring $Q I I$ is isomorphic to a cartesian product $F_{1} \times \cdots \times F_{r}$ of cyclotomic number fields. Note that the conjugation operation in $Q I I$ corresponds to the usual complex conjugation operation in each $F_{i}$. Let $E_{i}$ be the maximal real subfield of $F_{i}$. It follows easily from the Dirichlet unit theorem that for every unit $v$ in the ring of integers of $F_{i}$, there exists a power $v^{k}, k>0$, which belongs to the subfield $E_{i}$. Therefore some power $u^{k}$ of

$$
u \in Z \Pi \subset F_{1} \times \cdots \times F_{r}
$$

must belong to the invariant subring $E_{1} \times \cdots \times E_{r}$. This implies that $u^{k}$ is self-conjugate, and hence that $(\bar{u} / u)^{k}=1$; as was to be proved.

Bass has also proved a functorial version of Theorem 6.2. [To appear]. Two consequences are striking:

THEOREM 6.8. Let $\Gamma$ range over all cyclic subgroups of the finite group $\Pi$. Then the images

- This was proved by Kaplansky (unpublished), before Bass's more general results were obtained. 


$$
i_{*} \mathrm{Wh}(\Gamma) \subset \mathrm{Wh}(\Pi)
$$

together generate a subgroup of finite index in $\mathrm{Wh}(\Pi)$.

Consider next an orthogonal representation $\Pi \rightarrow O(n)$ of our finite group $\Pi$. This representation gives rise to a ring homomorphism

$$
h: Z \Pi \rightarrow M_{n}(R)
$$

and hence to an induced group homomorphism

$$
h_{*}: \bar{K}_{1}(Z \Pi) \rightarrow \bar{K}_{1} M_{n}(R) \cong R^{+} .
$$

(Compare \$1.10.) Since the group $R^{+}$has no elements of finite order, there is clearly a corresponding homomorphism

$$
\mathrm{Wh}(\Pi) \rightarrow R^{+}
$$

which will also be denoted by $h_{*}$.

ThEOREM 6.9. An element $\omega \in \mathrm{Wh}(\Pi)$ has finite order if and only if $h_{*}(\omega)=1$ for every irreducible orthogonal representation $h$ of the finite group II.

This follows easily from Bass [to appear].

Corollary 6.10. For any finite $\Pi$ and any $\omega \in \mathrm{Wh}(\Pi)$, the difference $\bar{\omega}-\omega$ is an element of finite order in $\mathrm{Wh}(\Pi)$.

Proof. This follows immediately from 6.7 and 6.8. Alternatively it follows easily from 6.9, making use of the fact that the determinant of a real matrix is equal to the determinant of its transpose.

7. Torsion for $\mathrm{CW}$-complexes. First consider the following situation. Let $(K, L)$ be a pair consisting of a finite, connected CWcomplex ${ }^{7} K$, and a subcomplex $L$ which is a deformation retract of $K$. The fundamental group $\pi_{1}(K)$ will be denoted briefly by $\Pi$. (Of course $\pi_{1} L \cong \pi_{1} K$.) The Whitehead torsion

$$
\tau(K, L) \in \mathrm{Wh}(\mathrm{II})
$$

can then be defined as follows.

For any pair $(X, Y)$ of $C W$-complexes the associated chain complex $C(X, Y)$ is defined by setting

$$
C_{p}(X, Y)=H_{p}\left(\left|X^{p} \cup Y\right|,\left|X^{p-1} \cup Y\right|\right),
$$

where $H$ denotes singular homology with integer coefficients, and where $\left|X^{p}\right|$ denotes the underlying topological space of the $p$-skele-

${ }^{7}$ See Whitehead [1949]. The reader may substitute "simplicial complex" for "CW-complex" and "simplex" for "cell" if he prefers. 
ton of $X$; i.e., the union of all cells of dimension $\leqq p$. This $p$ th chain group is free abelian with one generator for each $p$-cell of $X-Y$. Note that the homology group $H_{p} C(X, Y)$ of this chain complex is canonically isomorphic to the singular group $H_{p}(|X|,|Y|)$. (Compare §5.1.) These mutually isomorphic groups will be denoted briefly by $H_{p}(X, Y)$.

Now consider the universal covering complexes $\hat{K} \supset \hat{L}$ of $K$ and $L$. The fundamental group II will be identified with the group of covering transformations, so that each $\sigma \in \Pi$ determines a mapping

$$
\sigma:(\hat{K}, \hat{L}) \rightarrow(\hat{K}, \hat{L}) \text {. }
$$

Note that $\sigma$ is cellular. (That is it carries the $p$-skeleton into the $p$-skeleton for each $p$.) Hence each $\sigma \in \Pi$ determines a chain map

$$
\sigma_{\sharp}: C(\hat{K}, \hat{L}) \rightarrow C(\hat{K}, \hat{L}) .
$$

This action makes each chain group $C_{p}(\hat{K}, \hat{L})$ into a module over the integral group ring $Z \Pi$. Clearly the resulting chain module is $Z \Pi$-free with one generator for each $p$-cell of $K-L$. Since $K$ is finite it follows that $C(\hat{K}, \hat{L})$ is finitely generated over $Z \Pi$.

Thus we obtain a free chain complex

$$
C_{n}(\hat{K}, \hat{L}) \rightarrow C_{n-1}(\hat{K}, \hat{L}) \rightarrow \cdots \rightarrow C_{0}(\hat{K}, L)
$$

over $Z$ III. The homology groups $H_{i}(\hat{K}, \hat{L})$ of this complex are zero since $|\hat{L}|$ is a deformation retract of $|\hat{K}|$.

If we were given a preferred basis $c_{p}$ for each module $C_{p}(\hat{K}, \hat{L})$ then the torsion

$$
\tau C(\hat{K}, L) \in \bar{K}_{1}(Z \Pi)
$$

would be defined, as in $\$ 3$. But the geometry of the situation determines a class of preferred bases, as follows. Let $e_{1}, \cdots, e_{\alpha}$ denote the $p$-cells of $K-L$. For each $e_{i}$ choose a representative cell $\hat{e}_{i}$ of $\hat{K}$ lying over $e_{i}$. Furthermore choose an orientation, so that $\hat{e}_{i}$ determines a basis element of $C_{p}(\hat{K}, \hat{L})$, which we may also denote by $\hat{e}_{i}$. Then $c_{p}=\left(\hat{e}_{1}, \cdots, \hat{e}_{\alpha}\right)$ is the required basis for $C_{p}(\hat{K}, \hat{L})$.

Using these bases, the torsion $\tau C(\hat{K}, \hat{L})$ is apparently defined as an element of $\bar{K}_{1}(Z \Pi)$. However we have made an arbitrary choice of the representative cells $\hat{e}_{i}$; which leads to a certain arbitrariness in the resulting torsion. To eliminate the indeterminacy it is necessary to pass to the quotient group

$$
\mathrm{Wh}(\Pi)=\bar{K}_{1}(Z \Pi) / \text { image }(\Pi)
$$

which was studied in $\S 6$.

Definition. The image of $\tau C(\hat{K}, \hat{L})$ in the quotient group $\mathrm{Wh}(\Pi)$ is called the Whitehead torsion $\tau(K, L)$.

To justify this definition we must prove that $\tau(K, L)$ does not de- 
pend on the choice of $\hat{e}_{i}$. But if $\hat{e}_{i}$ is replaced by a different representative cell $\pm \sigma \not \hat{e}_{i}$ then a straightforward verification shows that $\tau C(\hat{K}, \hat{L})$ is replaced by

$$
\tau C(\hat{K}, \hat{L})-(-1)^{p}[\sigma] \in \bar{K}_{1}(Z \Pi) .
$$

Thus the difference $\pm[\sigma]$ belongs to image $(\Pi)$, and is annihilated when we pass to the quotient group $\mathrm{Wh}(I I)$. This completes the proof.

REMARK 1. In making use of the group $\mathrm{Wh}(\Pi)=\mathrm{Wh}\left(\pi_{1} K\right)$ we never need to worry about base points, since any inner automorphism of $\Pi$ will induce the identity automorphism of $\mathrm{Wh}(\Pi)$.

REMARK 2. Of course we can generalize the definition. Instead of assuming that $L$ is a deformation retract of $K$ it is sufficient to assume that $H_{*}(\hat{K}, \hat{L})$ is free over $Z \Pi$ with a preferred basis. Here $\hat{K}$ denotes the universal covering of $K$; and $\hat{L}$ denotes the appropriate subcomplex. As group $\Pi$ we must take $\pi_{1} K$, rather than $\pi_{1} L$. More generally II might be the group of covering transformations for any regular covering of $K$. With a little more effort one could even define a sharper invariant using the group $\pi_{1}(K-L)$ in place of $\pi_{1}(K)$. (Compare 7.2.)

Fundamental Unsolved Problem. We would like to assert that the torsion $\tau(K, L)$ is a topological invariant of the pair $(|K|,|L|)$, i.e., that it does not depend on the cell structure. However no one has been able to prove this, or to produce a counterexample. (Compare Milnor [1961].) The best result that one has is Whitehead's theorem that $\tau$ is invariant under subdivision:

Definition. A second CW-complex $X$ is a subdivision of $K$ if the underlying space $|X|$ is equal to $|K|$, and if each open cell of $X$ is contained in a (possibly higher dimensional) open cell of $K$, so that the identity map $K \rightarrow X$ is cellular. (Compare Figure 1.) Similarly the pair $(X, Y)$ is a subdivision of the pair $(K, L)$ if $X$ is a subdivision of $K$ and $Y$ is a subdivision of $L$.

Combinatorial Invariance Theorem 7.1. The torsion $\tau(K, L)$ is invariant under subdivision of the pair $(K, L)$.

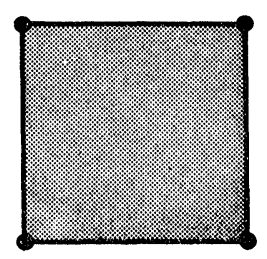

K

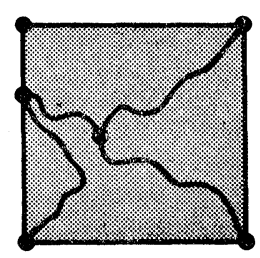

$\times$

Figure 1. Subdivision of a CW-complex. 
Our proof will depend on the machinery set up in $\$ 5$. (For a different proof see Whitehead [1950], Milnor [1961].) The geometrical kernel of the proof is contained in the following.

Lemma 7.2. Suppose that each component of $K-L$ is simply connected (where $L$ is a deformation retract of $K$ ). Then $\tau(K, L)=0$.

Proof. First suppose that $K-L$ has a single component $\Gamma$. Choose a representative component $\hat{\Gamma}$ of $\hat{K}-\hat{L}$. Clearly $\hat{\Gamma}$ projects homeomorphically onto $\Gamma$.
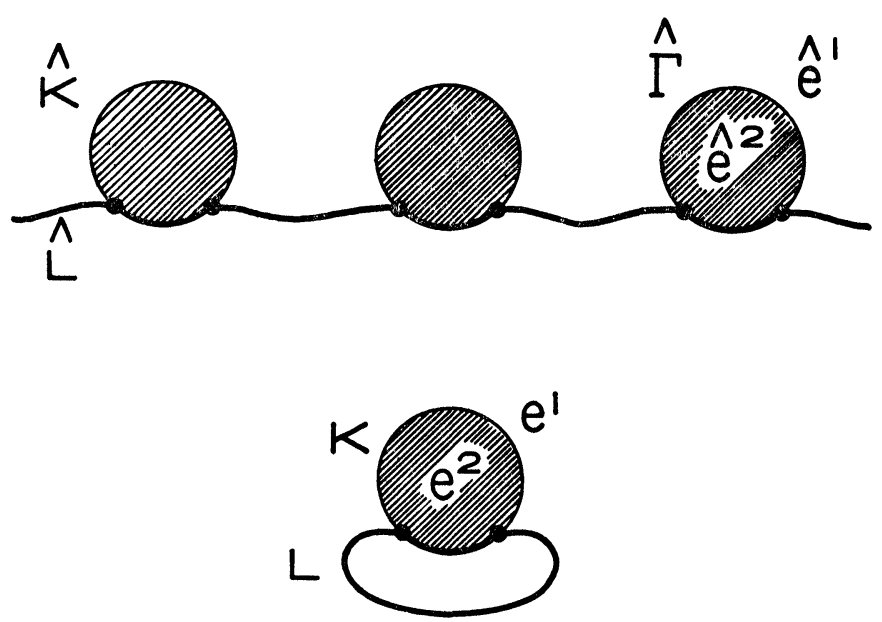

FIGURE 2. Judicious choice of representative cells in $\hat{K}$.

For each cell $e$ of $K-L$ choose the representative cell $\hat{e}$ as the unique cell in $\hat{\Gamma}$ which lies over $e$. (Compare Figure 2.) Now notice that no representative cell $\hat{e}^{k}$ of $\hat{K}-\hat{L}$ can be incident to a proper translate $\sigma \hat{e}^{k-1}, \sigma \neq 1$, of a representative cell. For $\sigma \hat{e}^{k-1}$ must be contained in a component $\sigma \hat{\Gamma}$ which is disjoint from $\hat{\Gamma}$. This means that the boundary $\partial \hat{e}^{k}$ can be expressed as a linear combination of representative $k-1$ cells with coefficients which are integers (rather than group ring elements). Thus in computing the torsion of the complex $C(\hat{K}, \hat{L})$ we need only work with the subring $Z \subset Z$ III. It follows that the torsion of $C(\hat{K}, \hat{L})$ belongs to the subgroup

$$
\bar{K}_{1} Z=0 \subset \bar{K}_{1}(Z \Pi) \text {. }
$$

Therefore $\tau(K, L)=0$.

If $K-L$ has several components the proof is essentially the same. It is only necessary to choose a representative component $\hat{\Gamma}_{i}$ lying over each component $\Gamma_{i}$ of $K-L$, and to choose representative cells $\hat{e} \subset \hat{\Gamma}_{i}$ as before. 
More generally suppose that $L$ is not a deformation retract of $K$.

Lemma 7.3. Suppose that $H_{*}(\hat{K}, \hat{L})$ is not zero, but is $Z \Pi$-free with preferred basis, where each basis element can be represented by a cycle lying in a single component of $\hat{K}-\hat{L}$. Assume as before that each component of $K-L$ is simply connected. Then $\tau(K, L)=0$.

Proof. Choose representative components $\hat{\Gamma}$ and representative cells $\hat{e}$ as before. Replace each preferred basis element $b \in H_{i}(\hat{K}, \hat{L})$ by the translate $\sigma b$, chosen so that $\sigma b$ is represented by a cycle lying within one of the representative components $\hat{\Gamma}$. Clearly this change of basis will not alter the torsion.

Again we see that $\tau(K, L)$ can be computed within the subring $\boldsymbol{Z} \subset Z I I$, and hence that $\tau(K, L)=0$.

Proof of Theorem 7.1. Let $C=C(\hat{X}, \hat{Y})$ be the chain complex associated with the subdivided pair; so that

$$
\tau(C) \mapsto \tau(X, Y) \in \mathrm{Wh}(\Pi) .
$$

We will define a sequence of subcomplexes

$$
C^{(0)} \subset C^{(1)} \subset C^{(2)} \subset \cdots \subset C^{(n)}=C
$$

so that the associated chain group

$$
\bar{C}_{\lambda}=H_{\lambda}\left(C^{(\lambda)} / C^{(\lambda-1)}\right)
$$

can be identified with $C_{\lambda}(\hat{K}, \hat{L})$. In particular the torsion $\tau(\bar{C})$ $\in \bar{K}_{1}(Z \Pi)$ will correspond to $\tau(K, L) \in W h(\Pi)$. We will then show that the hypotheses of $\$ 5.2$ are all satisfied. Thus Theorem 5.2 will imply that

$$
\tau(C)=\tau(\bar{C})
$$

and therefore that

$$
\tau(X, Y)=\tau(K, L)
$$

The construction follows. Let $X(p)$ denote the subcomplex of $X$ consisting of all cells of $X$ which are contained in the subspace $\left|K^{p} \cup L\right| \subset|X|$. Let $\hat{X}(p)$ denote the corresponding subcomplex of $\hat{X}$. Finally, let

$$
C^{(p)}=C(\hat{X}(p), \hat{Y}) .
$$

Then

$$
\begin{aligned}
& H_{i}\left(C^{(p)} / C^{(p-1)}\right)=H_{i}(\hat{X}(p), \hat{X}(p-1)) \\
\cong & H_{i}\left(\left|\hat{K}^{p} \cup \hat{L}\right|,\left|\hat{K}^{p-1} \cup \hat{L}\right|\right) .
\end{aligned}
$$

Clearly this group is zero for $i \neq p$, and is equal to $C_{p}(\hat{K}, \hat{L})$ for $i=p$. Hence the associated complex $\bar{C}$ can be identified with $C(\hat{K}, \hat{L})$. 
In order to apply $\S 5.2$, we must verify that the torsion

$$
\tau\left(C^{(p)} / C^{(p-1)}\right)=\tau C(\hat{X}(p), \hat{X}(p-1))
$$

is zero. But this follows from 7.3 since:

(1) each component of $|X(p)|-|X(p-1)|$ is a $p$-cell of the complex $K$, and therefore is simply connected; and

(2) each preferred generator for $H_{p}(\hat{X}(p), \hat{X}(p-1)) \cong C_{p}(\hat{K}, \hat{L})$ is represented by a cycle which lies in a single component of $|\hat{X}(p)|$ $-|\hat{X}(p-1)|$.

Hence we can apply 5.2 , and conclude that $\tau(X, Y)=\tau(K, L)$. This completes the proof of the Combinatorial Invariance Theorem.

To conclude this section we will discuss the torsion of a mapping cylinder, and the concept of "simple homotopy equivalence." First let us state an obvious consequence of $\S 3.1$.

Lemma 7.4. If $K \supset L \supset M$ where both $L$ and $M$ are deformation retracts of $K$, then

$$
\tau(K, M)=\tau(K, L)+i_{*} \tau(L, M),
$$

where $i: \pi_{1} L \cong \pi_{1} K$.

Next a familiar definition:

The mapping cylinder of a cellular mapping $f: X \rightarrow Y$ is defined to be the complex $M_{f}$ whose underlying space $\left|M_{f}\right|$ is obtained from $(|X| \times[0,1]) \cup|Y|$ by identifying each $(x, 1) \in|X| \times[1]$ with $f(x) \in|Y|$. (Compare Figure 3.) The cell structure is chosen in the
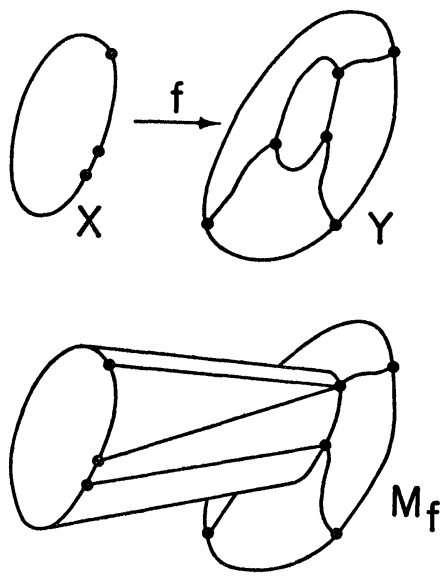

FIgURE 3. A mapping cylinder. 
obvious way so that $X(=X \times[0])$ and $Y$ are disjoint ${ }^{8}$ subcomplexes of $M_{f}$. Clearly $Y$ is a deformation retract of $M_{f}$.

Lemma 7.5. The torsion $\tau\left(M_{f}, Y\right)$ is zero.

Proof. Let $f(p): X^{p} \rightarrow Y$ denote the restriction of $f$ to the $p$-skeleton of $X$, so that

$$
Y=M_{f(-1)} \subset M_{f(0)} \subset M_{f(1)} \subset \cdots \subset M_{f(n)}=M_{f} .
$$

Then

$$
\tau\left(M_{f}, Y\right)=\sum_{p} \tau\left(M_{f(p)}, M_{f(p-1)}\right)
$$

by repeated application of 7.4. But each term on the right is zero by 7.2.

If $f$ happens to be a homotopy equivalence then clearly $X$ too is a deformation retract of $M_{f}$. However the torsion

$$
\tau\left(M_{f}, X\right) \in \mathrm{Wh}\left(\pi_{1} M_{f}\right)
$$

is not always zero.

Definition. For any cellular homotopy equivalence

$$
f: X \rightarrow Y
$$

the torsion $\tau(f)$ is defined to be that element of $\mathrm{Wh}\left(\pi_{1} Y\right)$ which corresponds to $\tau\left(M_{f}, X\right)$ under the natural isomorphism

$$
\mathrm{Wh}\left(\pi_{1} M_{f}\right) \cong \mathrm{Wh}\left(\pi_{1} Y\right) \text {. }
$$

If $\tau(f)=0$, then $f$ is called a simple homotopy equivalence.

Here are some of the basic properties of this concept.

Lemma 7.6. If $i: L \rightarrow K$ is an inclusion map, then $\tau(i)=\tau(K, L)$.

The proof is straightforward.

LEMmA 7.7. If $f_{0}$ and $f_{1}$ are homotopic then $\tau\left(f_{0}\right)=\tau\left(f_{1}\right)$.

Lemma 7.8. If $f: X \rightarrow Y$ and $g: Y \rightarrow Z$ are cellular homotopy equivalences, then

$$
\tau(g \circ f)=\tau(g)+g_{*} \tau(f) .
$$

It will be convenient to prove a more general statement which implies both 7.7 and 7.8. Let $h: X \rightarrow Z$ be any cellular mapping which is homotopic to $g \circ f$.

${ }^{8}$ This language is convenient; but can be unfortunate if (for example) $X$ is equal to $Y$. More precisely one should say that $M_{f}$ contains a "copy" of $X$ and a disjoint "copy" of $Y$. 
Proof that $\tau(h)=\tau(g)+g_{*} \tau(f)$. Choose a cellular homotopy

$$
X \times[0,1] \rightarrow Z
$$

between $h$ and $g \circ f$. (This is possible by Whitehead [1949, $\S 5(K)]$.) This homotopy can be pieced together with the mapping $g: Y \rightarrow Z$ to yield a cellular mapping

where

$$
H: M_{f} \rightarrow Z
$$

$$
H|X \times[0]=h, \quad H| Y=g .
$$

Note that the mapping cylinder $M_{H}$ contains the mapping cylinders

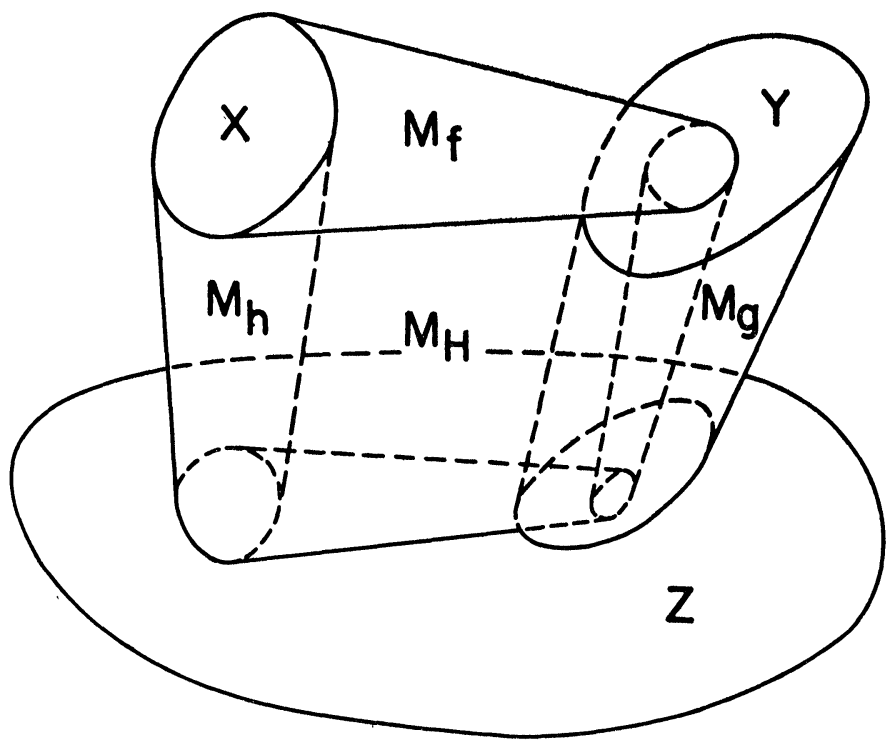

FIGURE 4. Schematic drawing of $M_{H}$.

of $f, g$ and $h$ as subcomplexes. (Compare Figure 4.) In fact we have the following diagram of inclusion maps.

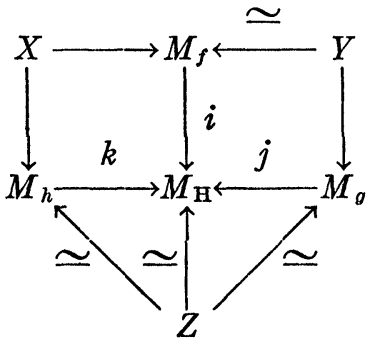


According to 7.5:

$$
\begin{gathered}
\tau\left(M_{f}, Y\right)=0, \quad \tau\left(M_{g}, Z\right)=0, \\
\tau\left(M_{H}, Z\right)=0 \quad \text { and } \quad \tau\left(M_{h}, Z\right)=0 .
\end{gathered}
$$

These facts have been indicated on the diagram by inserting the symbol $\simeq$ (for simple homotopy equivalence) on the appropriate arrows. Applying 7.4 to the second and third of these equations, we see that

Similarly

$$
\tau\left(M_{H}, M_{\theta}\right)=0 \text {. }
$$

$$
\tau\left(M_{H}, M_{h}\right)=0 \text {. }
$$

In other words $j$ and $k$ are simple homotopy equivalences. Now applying 7.4 to the right hand square we see that

$$
\tau\left(M_{H}, M_{f}\right)+i_{*} 0=0+j_{*} \tau\left(M_{g}, Y\right) .
$$

Similarly from the left hand square:

$$
0+k_{*} \tau\left(M_{h}, X\right)=\tau\left(M_{H}, M_{f}\right)+i_{*} \tau\left(M_{f}, X\right) \text {. }
$$

Hence

$$
k_{*} \tau\left(M_{h}, X\right)=j_{*} \tau\left(M_{g}, Y\right)+i_{*} \tau\left(M_{f}, X\right)
$$

Now applying the isomorphism

$$
\mathrm{Wh}\left(\pi_{1} M_{H}\right) \cong \mathrm{Wh}\left(\pi_{1} Z\right)
$$

to each of these three terms, we obtain the required equation

$$
\tau(h)=\tau(g)+g_{*} \tau(f)
$$

This completes the proof of 7.7 and 7.8.

REMARK. It follows from 7.7 that the torsion can be defined even for a homotopy equivalence $X \rightarrow Y$ which is not cellular. For Whitehead showed that any map between $\mathrm{CW}$-complexes is homotopic to a cellular map.

It is not however possible to dispense with the hypothesis that $X$ and $Y$ are finite complexes.

8. Change of rings: the $R$-torsion. This section will study an alternative form of torsion, to be called the "Reidemeister, Franz, de Rham real representation torsion," or briefly " $R$-torsion." This $R$-torsion has two advantages over the Whitehead torsion: It is more likely to be defined; and its value is an honest real number, instead of an element of an esoteric group. On the other hand the Whitehead 
torsion, when defined, is a sharper invariant.

First consider the general problem of changing rings. Let $C$ be a free complex of left $A$-modules, where each $C_{k}$ has preferred basis $c_{k}$. Let

$$
h: A \rightarrow A^{\prime}
$$

be a ring homomorphism. Then a free complex $C^{\prime}$ over $A^{\prime}$ is obtained by setting

$$
C_{k}^{\prime}=A^{\prime} \underset{A}{\otimes} C_{k}
$$

(using the homomorphism $h$ to make $A^{\prime}$ into a right $A$-module). Furthermore the basis

$$
c_{k}=\left(c_{k 1}, \cdots, c_{k \alpha}\right)
$$

for $C_{k}$ determines a basis

$$
c_{k}^{\prime}=\left(1 \otimes c_{k 1}, \cdots, 1 \otimes c_{k \alpha}\right)
$$

for $C_{k}^{\prime}$.

Hypothesis. Suppose that $C^{\prime}$ is acyclic (that is $H_{k} C^{\prime}=0$ for all $k$ ).

Then using the preferred bases $c_{k}^{\prime}$ the torsion $\tau\left(C^{\prime}\right) \in \bar{K}_{1} A^{\prime}$ is defined. This element will be denoted by $\tau_{h}(C)$ and called the torsion of $C$ associated with the representation $h$ of $A$.

For example if the original complex $C$ is acyclic then the new complex $C^{\prime}$ will certainly also be acyclic. Hence in this case both $\tau(C)$ and $\tau_{h}(C)$ are defined. However the relation

$$
\tau_{h}(C)=h_{*} \tau(C)
$$

shows that we have not really produced anything new in this case. The real interest lies rather in the fact that $\tau_{h}$ may be defined even when $\tau$ is not. Examples will be given presently.

The simplest choice for the ring $A^{\prime}$ is a field: say the field of complex numbers. However, if we are interested in noncommutative situations, then it is necessary to look at more general possibilities. This section will emphasize the possibility of using a real matrix ring for $A^{\prime}$.

Now for the geometry. As in $\$ 7$ consider pairs $(K, L)$ of finite $\mathrm{CW}$-complexes. We again assume that $K$ is connected, with fundamental group $\Pi$; but we no longer require that $L$ be a deformation retract of $K$. As before the chain complex $C(\hat{K}, \hat{L})$ is free over $Z \Pi$ with preferred bases. We will discuss two different procedures for changing the ring, so as to obtain a more manageable torsion. A third procedure will be discussed in $\$ 12$. 
First procedure. Consider a homomorphism

$$
h: \Pi \rightarrow F^{*}
$$

from $I I$ to the multiplicative group of a field $F$, say the field of complex numbers. Then $h$ gives rise to a ring homomorphism

$$
Z \mathrm{II} \rightarrow F
$$

which will also be denoted by $h$. Using $h$ we can form the chain complex

$$
C^{\prime}=F \underset{Z I I}{\otimes} C(\hat{R}, \hat{L})
$$

over $F$. If $H_{*}\left(C^{\prime}\right)=0$ then the torsion

$$
\tau\left(C^{\prime}\right) \in \bar{K}_{1} F=F^{*} /\{ \pm 1\}
$$

is more or less well defined. To be more precise, this torsion is well defined providing that we pass to the quotient group

$$
\bar{K}_{1} F / \text { image }(\Pi)=F^{*} /\{ \pm 1\} h(\Pi) \text {. }
$$

As in $\$ 7$, torsion is invariant under subdivision. We will use the notation

$$
\Delta_{h}(K, L) \in F^{*} / \pm h(\Pi)
$$

or

$$
\pm h(\Pi) \Delta_{h}(K, L) \subset F^{*}
$$

for this torsion. ${ }^{9}$ The use of this new symbol $\Delta$ will help to distinguish $\Delta_{h}(K, L)$ from the Whitehead torsion $\tau(K, L)$. Also the new notation will emphasize that $\Delta$ belongs to a multiplicative rather than an additive group.

This is the original construction as used by Reidemeister and Franz. It is particularly effective if $\Pi$ is a finite cyclic group, and $F$ is the field of complex numbers. But it can also be used in other cases:

EXAMPLE 1. Let $K$ be the circle $S^{1}$ and let $L$ be vacuous. The Whitehead torsion $\tau\left(S^{1}, \phi\right)$ cannot be defined, since the module $H_{0}\left(\hat{S}^{1}\right)$ is not zero, and is not $Z$ III-free. However if the homomorphism

$$
h: \pi_{1} S^{1} \rightarrow F^{*}
$$

maps a generator into the field element $f \neq 1$, then computation shows

- Caution. Due to a difference in sign conventions, this $\Delta$ corresponds to $\Delta^{-1}$ in de Rham [1950] and Milnor [1961], [1962]. 
that the associated torsion

$$
\Delta_{h}\left(S^{1}\right) \in F^{*} / \pm h(\mathrm{I})
$$

is well defined, and is equal to $1-f$ (up to multiplication by plus or minus a power of $f$ ).

ExAMPLE 2. Let $X$ be obtained from the 3 -sphere by boring out the interior of a knotted tube. Let

$$
h: \pi_{1}(X) \rightarrow F^{*}
$$

map each loop with linking number +1 into the field element $f \neq 1$. Then $\Delta_{h}(X)$ is well defined, and is equal to $(1-f) / A(f)$ (up to multiplication by $\left.\pm f^{i}\right)$, where $A(t)$ denotes the Alexander polynomial of the knot. (Compare Milnor [1962].)

In general, if we want to work with a noncommutative group II, it is better to allow representations of $\Pi$ by $n \times n$ matrices, with $n>1$. Once we have made this step, the complex numbers no longer seem to have any advantage over the real numbers.

Second Procedure. Let $h: \Pi \rightarrow O(n)$ be an orthogonal representation of the group II. Then $h$ extends to a unique ring homomorphism from $Z \Pi$ to the ring $M_{n}(R)$ of all real $n \times n$ matrices. Using $h$ we can form the complex

$$
C^{\prime}=M_{n}(R) \underset{\Pi}{\otimes} C(\hat{K}, \hat{L}) .
$$

If this complex happens to be acyclic, then the torsion

$$
\tau\left(C^{\prime}\right) \in \bar{K}_{1} M_{n}(R)=\bar{K}_{1} R
$$

is defined.

The group $\bar{K}_{1} R$ can be identified either with the multiplicative group $R^{+}$or with the additive group $R$, using the correspondence

$$
\left(a_{i j}\right)|\rightarrow| \operatorname{det}\left(a_{i j}\right) \mid \text { or } \log \left|\operatorname{det}\left(a_{i j}\right)\right|
$$

respectively. It doesn't really matter which identification is used; but the multiplicative notation is customary in this context. We will again use the notation $\Delta$ instead of $\tau$ to emphasize the switch to a multlplicative notation.

Definition. The positive real number corresponding to $\tau\left(C^{\prime}\right)$ $\in \bar{K}_{1} R$ will be denoted by $\Delta_{h}(K, L)$, and called the $R$-torsion of $(K, L)$ with respect to $h$. The $R$-torsion also is invariant under subdivision.

This $R$-torsion is completely well defined. In particular, there is no ambiguity coming from the lack of uniqueness of representative cells in the universal covering space. For if the $p$-cell $\hat{e}$ of $\hat{K}$ is replaced by 
a translate $\sigma \hat{e}$, then $\Delta_{h}(K, L)$ will be multiplied or divided by $|\operatorname{det} h(\sigma)|$ according as $p$ is odd or even. But this determinant is \pm 1 , since $h(\sigma)$ is orthogonal.

Let us compare $R$-torsions with Whitehead torsions. Recall from $\$ 6$ that any orthogonal representation

$$
h: \Pi \rightarrow O(n)
$$

determines a homomorphism

$$
h_{*}: \mathrm{Wh}(\Pi) \rightarrow \bar{K}_{1} R \cong R^{+} .
$$

THEOREM 8.1. Suppose that the Whitehead torsion invariant

$$
\tau(K, L) \in \mathrm{Wh}(\Pi)
$$

is defined; and suppose that $\Pi$ is a finite group. Then $\tau(K, L)$ is an element of finite order in $\mathrm{Wh}(\Pi)$ if and only if $\Delta_{h}(K, L)=1$ for all possible orthogonal representations $h$ of $\Pi$. If $\Pi$ is finite abelian, then $\tau=0$ if and only if $\Delta_{h}=1$ for all possible $h$.

PRoof. This follows immediately from $\S 6.9$ and $\$ 6.4$ together with the identity

$$
\Delta_{h}(K, L)=h_{*} \tau(K, L) .
$$

REMARK. Of course one need only consider representations $h$ which are irreducible. Furthermore one need consider only "irrational" representations. For if $h$ is equivalent to a representation by rational matrices, then it is equivalent to a representation by integer matrices; and hence $h_{*}$ is trivial.

As a final illustration of $R$-torsions, consider the following.

By a spherical Clifford-Klein manifold $M^{n}$ will be meant a manifold of the form $S^{n} / \Pi$ where $\Pi$ is a finite fixed-point free group of orthogonal motions of the $n$-sphere. (If $\Pi$ is cyclic then $S^{n} / \Pi$ is called a lens space.) It will be shown in $\S 9$ that torsion can be defined for such smooth manifolds.

Theorem 8.2 (DE Rham). A spherical Clifford-Klein manifold is determined up to isometry by its fundamental group $\Pi$ together with the collection $\left\{\Delta_{h}\left(M^{n}\right)\right\}$ of $R$-torsion invariants.

Hence two such manifolds which are diffeomorphic must actually be isometric.

For the proof the reader is referred to de Rham [1950]. In the case of a lens space this result is due to Franz [1935], and will be studied further in $\$ 12$. 
9. Torsion for smooth manifolds. Let $W$ be a smooth $\left(=C^{\infty}\right)$ manifold whose boundary is the disjoint union $M \cup M^{\prime}$ of two closed submanifolds. If $W$ is compact, connected, and both $M$ and $M^{\prime}$ are deformation retracts of $W$, then the triad $\left(W ; M, M^{\prime}\right)$ is called an $h$-cobordism.

If the fundamental group

$$
\Pi=\pi_{1} M \cong \pi_{1} W \cong \pi_{1} M^{\prime}
$$

is trivial, and the dimension $n$ of $W$ is $\geqq 6$, then a theorem of Smale [1962] asserts that $W$ is necessarily a product:

$$
W \approx M \times[0,1] \text {. }
$$

We will be interested in the case when $\Pi$ is nontrivial. According to a theorem of Stallings [1965 $]$, the $h$-cobordism is then completely determined by $M$, together with the torsion invariant $\tau(W, M) \in \mathrm{Wh}(\Pi)$. (See $\$ 11$.

To define this torsion, one must somehow construct an algebraic chain complex out of the geometric situation. Three ways of doing this are known to me:

(1) The construction can be based on Whitehead's theory of $C^{1}$-triangulations.

(2) It can be based on the Smale-Morse theory of handlebodies (or of nondegenerate functions).

(3) It can be based on the nerve of a covering by geodesically convex open sets (de Rham [1950]).

We will study only the first two possibilities. The first is the easiest. Let

$$
t:(|K|,|L|) \rightarrow(W, M)
$$

be a $C^{1}$-triangulation. (See Munkres [1963]. Here $|K|$ denotes the underlying topological space of the simplicial complex $K$.) Now define $\tau(W, M)$ to be $t_{*} \tau(K, L)$, where

$$
t_{*}: \mathrm{Wh}\left(\pi_{1}|K|\right) \rightarrow \mathrm{Wh}\left(\pi_{1} W\right)
$$

denotes the isomorphism induced by $t$.

Lemma 9.1. This element $\tau(W, M)$ does not depend on the choice of the $C^{1}$-triangulation $t$.

Proof. Let $u:(|X|,|Y|) \rightarrow(W, M)$ be a second $C^{1}$-triangulation. By a fundamental theorem of Whitehead, there exist rectilinear subdivisions $\left(K^{\prime}, L^{\prime}\right)$ of $(K, L)$ and $\left(X^{\prime}, Y^{\prime}\right)$ of $(X, Y)$ which are isomorphic to each other under an isomorphism 


$$
\phi:\left(K^{\prime}, L^{\prime}\right) \rightarrow\left(X^{\prime}, Y^{\prime}\right)
$$

of simplicial complexes. Furthermore, given $\epsilon>0$, these subdivisions can be chosen so that the composition

$$
u|\phi| t^{-1}:(W, M) \rightarrow(W, M)
$$

is an $\epsilon$-approximation to the identity. (See Munkres $\$ 10.5$.)

If $\epsilon$ is sufficiently small, then clearly $u_{*}|\phi|{ }_{*} t_{*}^{-1}$ must be the identity automorphism of $\mathrm{Wh}(\Pi)$. Hence

$$
\begin{aligned}
t_{*} \tau(K, L) & =t_{*} \tau\left(K^{\prime}, L^{\prime}\right)=u_{*}|\phi|_{*} \tau\left(K^{\prime}, L^{\prime}\right) \\
& =u_{*} \tau\left(X^{\prime}, Y^{\prime}\right)=u_{*} \tau(X, Y) ;
\end{aligned}
$$

which completes the proof.

Next we will discuss the definition based on Smale-Morse theory. Let $W$ be a smooth manifold with boundaries $M$ and $M^{\prime}$. A smooth function

$$
f: W \rightarrow[a, b]
$$

is called a Morse function if $f^{-1}(a)=M, f^{-1}(b)=M^{\prime}$; and if the critical points of $f$ are interior and nondegenerate. Now suppose that $a=-\frac{1}{2}$, $b=n+\frac{1}{2}$. Definition. $f$ is a nice function (or self-indexing function) if all critical points of index $\lambda$ lie in the subset $f^{-1}(\lambda) \subset W$. A theorem of Smale asserts that such functions always exist.

Defining $W^{(\lambda)}=f^{-1}\left[a, \lambda+\frac{1}{2}\right]$ it then follows that

$$
M=W^{(-1)} \subset W^{(0)} \subset W^{(1)} \subset \cdots \subset W^{(n)}=W,
$$

where each $W^{(\lambda)}$ is a smooth manifold which contains precisely the critical points of index $\leqq \lambda$.

Given such a nice function $f$ we will define the torsion $\tau(W, M, f)$ relative to $f$. It will then be established that this coincides with our earlier definition of $\tau(W, M)$, and hence does not actually depend on $f$.

Let $\hat{W}$ denote the universal covering manifold of $W$, and $\hat{f}: \hat{W} \rightarrow[a, b]$ the map corresponding to $f$. Then $\hat{W}^{(\lambda)}=\hat{f}^{-1}\left[a, \lambda+\frac{1}{2}\right]$ is the portion of $\hat{W}$ which lies over $W^{(\lambda)}$.

Lemma 9.2. The singular homology group $\boldsymbol{H}_{i}\left(\hat{W}^{(\lambda)}, \hat{W}^{(\lambda-1)}\right)$ is zero for $i \neq \lambda$ and is free abelian for $i=\lambda$, with one generator for each critical point of index $\lambda$ in $\hat{W}$.

PRoof. It is well known that $W^{(\lambda)}$ contains as deformation retract a subset $W^{(\lambda-1)} \cup e_{1} \cup \cdots \cup e_{\alpha}$ where $e_{1}, \cdots, e_{\alpha}$ are disjoint $\lambda$-cells which pass through the critical points $x_{1}, \cdots, x_{\alpha}$ of index $\lambda$ in $W$, 
and which are attached to $W^{(\lambda-1)}$ only along their boundaries. (See Milnor $[1963, \S 3.3]$.) Hence the covering space $\hat{W}^{(\lambda)}$ has a similar description. However each $\lambda$-cell $e_{i}$ is covered by a collection of disjoint $\lambda$-cells $\sigma \hat{e}_{i}$ where $\sigma$ ranges over the group $\Pi$ of covering transformations, and where $\hat{e}_{i}$ denotes a single representative cell. Precisely one cell $\sigma \hat{e}_{i}$ passes through each of the points $\sigma \hat{x}_{i}$ lying over $x_{i}$ in $\hat{W}$. This completes the proof.

If we take account of the action of $\Pi$ then it is evident that $H_{\lambda}\left(\hat{W}^{(\lambda)}, \hat{W}^{(\lambda-1)}\right)$, becomes a free module over the group ring $Z \Pi$, with $\alpha$ generators. Setting $H_{\lambda}\left(\hat{W}^{(\lambda)}, \hat{W}^{(\lambda-1)}\right)$ equal to $\bar{C}_{\lambda}$ we have thus constructed a free chain complex

$$
\bar{C}_{n} \rightarrow \bar{C}_{n-1} \rightarrow \cdots \rightarrow \bar{C}_{0}
$$

over $Z \Pi$. The homology of this chain complex is isomorphic to $\boldsymbol{H}_{*}(\hat{W}, \hat{M})$ (compare $\S 5$ ) and hence is zero; since $\hat{M}$ is a deformation retract of $\hat{W}$.

The above construction also yields a preferred basis for $\bar{C}_{\lambda}$. [The procedure can be described more invariantly as follows. For each critical point $x_{i}$ in $f^{-1}(\lambda)$ choose a covering critical point $\hat{x}_{i}$. Now if $U$ is a small neighborhood of $\hat{x}_{i}$ note that the group

$$
H_{\lambda}\left(U \cap \hat{f}^{-1}[a, \lambda], U \cap \hat{f}^{-1}[a, \lambda)\right)
$$

is infinite cyclic, and maps monomorphically into

$$
\boldsymbol{H}_{\lambda}\left(\hat{f}^{-1}[a, \lambda], \hat{f}^{-1}[a, \lambda)\right) \cong \boldsymbol{H}_{\lambda}\left(\hat{W}^{(\lambda)}, \hat{W}^{(\lambda-1)}\right)=\bar{C}_{\lambda} .
$$

Thus the choice of $\hat{x}_{i}$ determines a basis element for $\bar{C}_{\lambda}$ up to sign.] Just as in $\$ 7$ the torsion $\tau \bar{C}$ is defined, apparently as an element of $\bar{K}_{1} Z \Pi$. But if we alter the choice of representatives $\hat{x}_{i}$, then, as in $\S 7$, this torsion will be altered by an element of the subgroup

$$
\text { image(I) } \subset \bar{K}_{1} Z \Pi \text {. }
$$

Hence the image of $\tau \bar{C}$ in the quotient group $W h(\Pi)=\bar{K}_{1} Z \Pi /$ image(II) is independent of the choice of representatives. This image will be denoted by $\tau(W, M ; f) \in \mathrm{Wh}(\Pi)$.

Theorem 9.3. If $t:(|K|,|L|) \rightarrow(W, M)$ is a $C^{1}$-triangulation then

$$
\tau(W, M ; f)=t_{*} \tau(K, L) .
$$

Hence $\tau(W, M ; f)=\tau(W, M)$ does not depend on the choice of nice function $f$.

REMARK. A corresponding theorem holds for $R$-torsions.

Proof of 9.3. We are at liberty to choose whatever $C^{1}$-triangula- 
tion we please. In particular we can choose the triangulation so that each $W^{(\lambda)}$ corresponds to a subcomplex $K^{(\lambda)} \subset K$. (Munkres $\S 10.6$.) It will be convenient to suppress the map $t$ by identifying $|K|$ with its image $t|K|=W$.

Thus the chain complexes $C^{(\lambda)}=C\left(\hat{K}^{(\lambda)}, \hat{L}\right)$ yield a filtration

$$
C^{(0)} \subset C^{(1)} \subset \cdots \subset C^{(n)}=C(\hat{K}, \hat{L}) \text {. }
$$

The relative homology groups $H_{i} C^{(\lambda)} / C^{(\lambda-1)}$ can be identified with $H_{i}\left(\hat{W}^{(\lambda)}, \hat{W}^{(\lambda-1)}\right)$, and hence satisfy the hypotheses of $\S 5$. Furthermore we have used the geometry to choose bases for the free modules

$$
\bar{C}_{\lambda}=H_{\lambda} C^{(\lambda)} / C^{(\lambda-1)}=H_{\lambda}\left(\hat{W}^{(\lambda)}, \hat{W}^{(\lambda-1)}\right) .
$$

We will prove:

Lemma 9.4. For each quotient complex $C^{(\lambda)} / C^{(\lambda-1)}$ the torsion $\tau C^{(\lambda)} / C^{(\lambda-1)} \in \mathrm{Wh}(\Pi)$ is zero.

Using the Algebraic Subdivision Theorem of $\$ 5$, this will prove that $\tau \vec{C}=\tau(W, M ; f)$ is equal to $\tau C=t_{*} \tau(K, L)$; which will prove the theorem.

Proof of Lemma. Let us recall something of the geometry of the region $f^{-1}\left[\lambda-\frac{1}{2}, \lambda+\frac{1}{2}\right]$ which contains the critical points of index $\lambda$. (Compare Milnor [1963, §3] and [1965].) It will be convenient to set

$$
X=f^{-1}\left[\lambda-\frac{1}{2}, \lambda+\frac{1}{2}\right], \quad B=f^{-1}\left[\lambda-\frac{1}{2}\right], \quad B^{\prime}=f^{-1}\left[\lambda+\frac{1}{2}\right]
$$

We must prove that $\tau(X, B)=0$.

Through each critical point $x_{i}$ one can construct a "left hand cell" $e_{i}^{\lambda}$ which meets $B$ in the sphere $\partial e_{i}^{\lambda}$, and a corresponding "right hand cell," which we will denote by $\epsilon_{i}^{n-\lambda}$, and which meets $B^{\prime}$ in a corre-

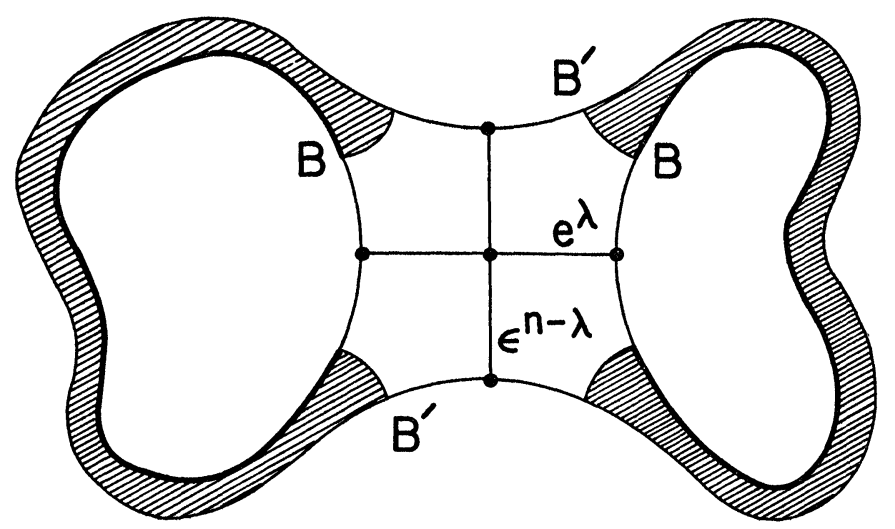

Figure 5. The "left and right-hand cells" for a critical point of index $\lambda=1$ on a surface. 
sponding sphere $\partial \epsilon_{i}^{n-\lambda}$. These cells are all disjoint except that $e_{i}^{\lambda} \cap \epsilon_{i}^{n-\lambda}=x_{i}$. Using the trajectories of a suitable vector field one can show that the two boundary manifolds

$$
B-\partial e_{1}^{\lambda}-\cdots-\partial e_{\alpha}^{\lambda}, \quad B^{\prime}-\partial \epsilon_{1}^{n-\lambda}-\cdots-\partial \epsilon_{\alpha}^{n-\lambda}
$$

are diffeomorphic. In fact the entire region

$$
X-\left(e_{1}^{\lambda} \cup{ }_{\epsilon_{1}^{n-\lambda}}^{n-\lambda}\right)-\cdots-\left(e_{\alpha}^{\lambda} \cup \epsilon_{\alpha}^{n-\lambda}\right)
$$

is diffeomorphic with the product

$$
\left(B-\partial e_{1}^{\lambda}-\cdots-\partial e_{\alpha}^{\lambda}\right) \times\left[\lambda-\frac{1}{2}, \lambda+\frac{1}{2}\right] .
$$

Let $B_{0}$ denote the manifold obtained from $B$ by removing an open tubular neighborhood of each $\partial e_{i}^{\lambda}$; and let

$$
X_{0} \approx B_{0} \times\left[\lambda-\frac{1}{2}, \lambda+\frac{1}{2}\right]
$$

denote the corresponding subset of $X$. (Compare Figure 5, in which $X_{0}$ is shaded and $B_{0}$ is emphasized.) We may assume that $X_{0}$ is triangulated as a product. (Compare the proof in Munkres [1963, \$10.6].)

Now we will apply two lemmas from $\S 7$. We have

$$
\tau(X, B)=\tau\left(X_{0} \cup B, B\right)+\tau\left(X, X_{0} \cup B\right)
$$

by Theorem 3.2. (To be accurate here, each of the symbols $X, X_{0}, B$ should be replaced by the symbol for the corresponding simplicial complex.) But the last term is zero by Lemma 7.3 since each component of $X-X_{0}-B$ is clearly contractible. The first term on the right is zero by Lemma 7.5 since $X_{0}$ is a product: $X_{0} \approx B_{0} \times\left[\lambda-\frac{1}{2}\right.$, $\lambda+\frac{1}{2}$ ]. (Such a product is clearly an example of a mapping cylinder. Furthermore the product triangulation of $X_{0}$ can be regarded as a subdivision of the product cell structure which was used in the discussion of mapping cylinders.)

Thus $\tau(X, B)=\tau C^{(\lambda)} / C^{(\lambda-1)}$ is zero; which completes the proof.

10. The duality theorem. The following basic theorem has been proved by Mazur [1963], Barden [1963] and Stallings. We will not try to give a proof.

$s$-Cobordism Theorem. Let $\left(W ; M, M^{\prime}\right)$ be an h-cobordism of dimension $n \geqq 6$. Then the torsion $\tau(W, M)$ vanishes if and only if $W$ is diffeomorphic to the product $M \times[0,1]$.

Suppose that we interchange the roles of $M$ and $M^{\prime}$. Clearly we can also say that $\tau\left(W, M^{\prime}\right)$ is zero if and only if $W$ is a product. This 
suggests that there should be a formula relating $\tau(W, M)$ and $\tau\left(W, M^{\prime}\right)$.

Duality Theorem. For any orientable h-cobordism $\left(W ; M, M^{\prime}\right)$ of dimension $n$ we have

$$
\tau\left(W, M^{\prime}\right)=(-1)^{n-1} \bar{\tau}(W, M),
$$

where $\bar{\tau}$ denotes the conjugate of $\tau$.

(For the definition of "conjugate" see §6.)

The proof will depend on a comparison between the nice function $f$ on $W$ and the "dual" nice function $n-f$.

As in $\S 9$, through each critical point $x_{i}$ of index $\lambda$ we can construct a "left-hand cell"

$$
e_{i}^{\lambda} \subset f^{-1}\left[\lambda-\frac{1}{2}, \lambda\right]
$$

and a right hand cell

$$
\epsilon_{i}^{n-\lambda} \subset f^{-1}\left[\lambda, \lambda+\frac{1}{2}\right]
$$

These are to be oriented so that the intersection number $e_{i}^{\lambda} \cdot \epsilon_{i}^{n-\lambda}$ is +1 . Furthermore we can cover $e_{i}^{\lambda}$ and $\epsilon_{i}^{n-\lambda}$ by representative cells in the universal covering space so that $\hat{e}_{i}^{\lambda} \cdot \hat{\epsilon}_{i}^{n-\lambda}=1$.

If we think of $\hat{e}_{i}^{\lambda}$ as a basis element for the chain group

$$
\bar{C}_{\lambda}=H_{\lambda}\left(\hat{W}^{(\lambda)}, \hat{W}^{(\lambda-1)}\right)
$$

then the boundary homomorphism $\partial: \bar{C}_{\lambda+1} \rightarrow \bar{C}_{\lambda}$ can be described by a matrix of "incidence numbers" $a_{k i} \in Z \Pi$, where

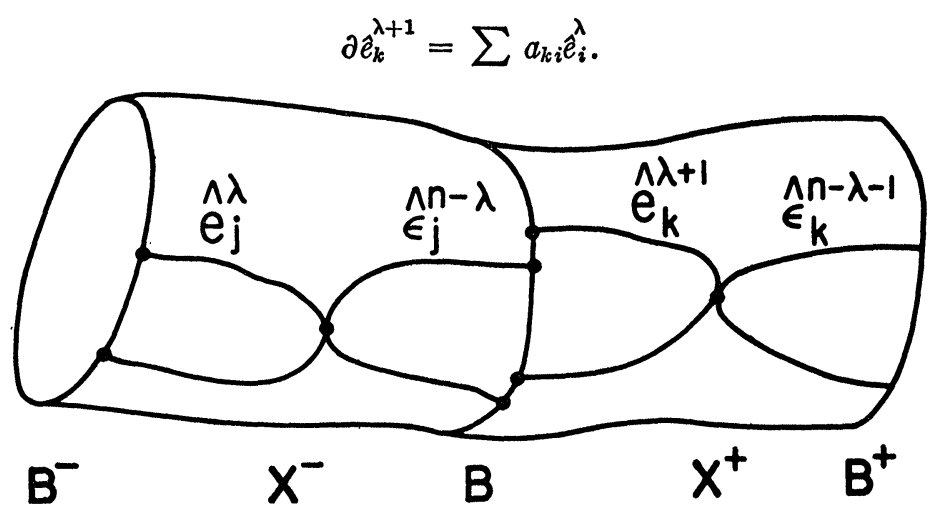

FIGURE 6. A highly schematic drawing of left and right hand cells for critical points of index $\lambda$ and $\lambda+1$.

Similarly, using the dual function $n-f$, we can define incidence numbers between the right hand cells. 
DuAlity Lemma. The incidence number between $\hat{\epsilon}_{i}^{n-\lambda}$ and $\hat{\epsilon}_{\mathrm{k}}^{n-\lambda-1}$ is equal to the conjugate $\bar{a}_{k i}$ of the incidence number between $\hat{e}_{\boldsymbol{z}}^{\lambda+1}$ and $\hat{e}_{i}^{\lambda}$, multiplied by a sign $(-1)^{\lambda+1}$ which depends only on the dimensions.

The proof will depend on some identities involving intersection numbers. It will be convenient to set

$$
\begin{gathered}
B^{-}=\hat{f}^{-1}\left[\lambda-\frac{1}{2}\right], \quad B=\hat{f}^{-1}\left[\lambda+\frac{1}{2}\right], \quad B^{+}=\hat{f}^{-1}\left[\lambda+\frac{3}{2}\right], \\
X^{-}=\hat{f}^{-1}\left[\lambda-\frac{1}{2}, \lambda+\frac{1}{2}\right], \quad X^{+}=\hat{f}^{-1}\left[\lambda+\frac{1}{2}, \lambda+\frac{3}{2}\right] .
\end{gathered}
$$

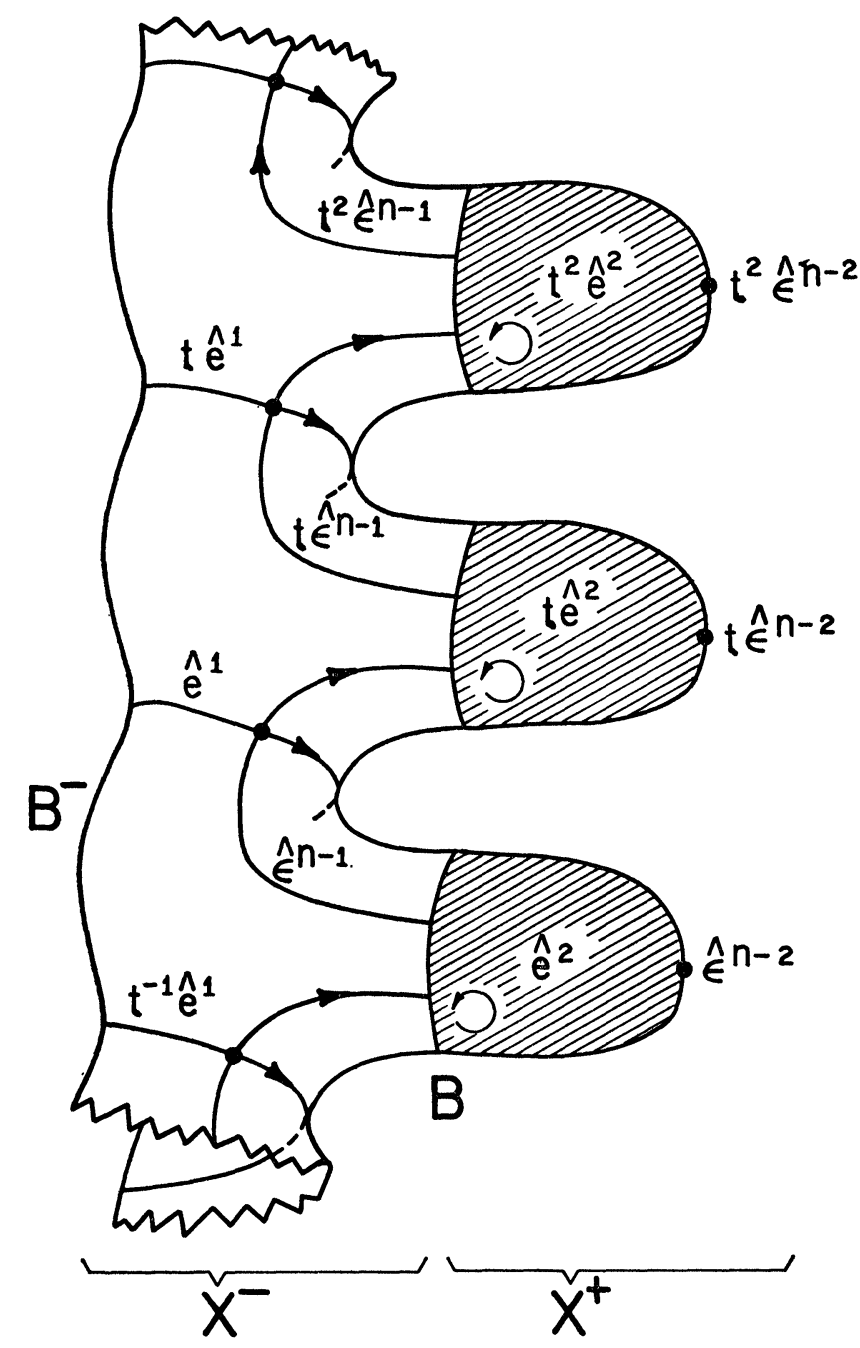

FIGURE 7. A more realistic drawing. (With $n=2$. Note that $B^{+}$is vacuous.) The boundary formulas $\partial \hat{e}^{2}=\left(t^{-1}-1\right) \hat{e}^{1}, \partial \hat{\epsilon}^{n-1}=(t-1) \hat{\epsilon}^{n-2}$ illustrate the Duality Lemma. 
(Compare Figures 6 and 7.) We will show that the matrix $\left(a_{k j}\right)$ can be described in terms of intersection numbers, either within $X^{-}$or within the middle manifold $B$.

Note that the homomorphism $\bar{\partial}: \bar{C}_{\lambda+1} \rightarrow \bar{C}_{\lambda}$ can be expressed as $\bar{\partial}=\iota * \partial$ where

$$
\begin{gathered}
\partial: \bar{C}_{\lambda+1}=H_{\lambda+1}\left(X^{+}, B\right) \rightarrow H_{\lambda} B, \\
\iota *: H_{\lambda} B \rightarrow H_{\lambda}\left(X^{-}, B^{-}\right)=\bar{C}_{\lambda} .
\end{gathered}
$$

(Here $\iota$ denotes the inclusion map $(B, \phi) \rightarrow\left(X^{-}, B^{-}\right)$.)

Let us compute the coefficient of $\sigma$ in the group ring element $a_{k j}$. In other words let us compute the coefficient of $\sigma \hat{e}_{j}^{\lambda}$ in $\partial \hat{e}_{k}^{\lambda+1}$. Clearly the element $\sigma \hat{\epsilon}_{j}^{n-\lambda}$ of $H_{n-\lambda}\left(X^{-}, B\right)$ has intersection number +1 with $\sigma \hat{e}_{j}^{\lambda}$, but has intersection number 0 with all of the other basis elements $\rho \hat{e}_{i}^{\lambda}$. This gives us the identity

$$
\begin{aligned}
& \text { coefficient of } \sigma \text { in } a_{k j} \\
& =\text { coefficient of } \sigma \hat{e}_{j}^{\lambda} \text { in } \bar{\partial} \hat{e}_{k}^{\lambda+1} \\
& =\left(\bar{\partial} \hat{e}_{k}^{\lambda+1}\right) \cdot\left(\sigma \hat{\epsilon}_{j}^{n-\lambda}\right) .
\end{aligned}
$$

Now suppose that $B$ is oriented as part of the boundary of $X^{-}$. Then for any elements

$$
u \in H_{\lambda} B, \quad v \in H_{n-\lambda}\left(X^{-}, B\right)
$$

the identity

$$
\iota * u \cdot v=(-1)^{\lambda} u \cdot \partial^{\prime} v
$$

is easily verified (where the first intersection number is evaluated within $X^{-}$and the second is evaluated within $B$ ). Setting

$$
\iota_{*} u=\iota_{*} \partial \hat{e}_{k}^{\lambda+1}=\bar{\partial} \hat{e}_{k}^{\lambda+1}, \quad v=\sigma \hat{\epsilon}_{j}^{n-\lambda}
$$

this yields the formula

$$
\begin{aligned}
& \text { coefficient of } \sigma \text { in } a_{k j} \\
& \quad=(-1)^{\lambda}\left(\partial \hat{\epsilon}_{k}^{\lambda+1}\right) \cdot\left(\sigma \partial^{\prime} \hat{\epsilon}_{j}^{n-\lambda}\right) .
\end{aligned}
$$

Now let us look at the corresponding matrix $\left(a_{j k}^{\prime}\right)$ for the dual homomorphism

$$
\bar{\partial}^{\prime}=\iota *^{\prime} \partial^{\prime}: H_{n-\lambda}\left(X^{-}, B\right) \rightarrow H_{n-\lambda-1}\left(X^{+}, B^{+}\right) .
$$

Similar arguments show that 


$$
\begin{aligned}
& \text { coefficient of } \sigma \text { in } a_{j k}^{\prime} \\
& =\text { coefficient of } \sigma \hat{\epsilon}_{k}^{n-\lambda-1} \text { in } \iota *^{\prime} \partial_{*}^{\prime} \hat{\epsilon}_{j}^{n-\lambda} \\
& =\left(\sigma \hat{e}_{k}^{\lambda+1}\right) \cdot\left(\iota \iota^{\prime} \partial^{\prime} \hat{\epsilon}_{j}^{n-\lambda}\right) \\
& =-\left(\partial \sigma \hat{e}_{k}^{\lambda+1}\right) \cdot\left(\partial^{\prime} \hat{\epsilon}_{j}^{n-\lambda}\right) \\
& =-\left(\partial \hat{e}_{k}^{\lambda+1}\right) \cdot\left(\sigma^{-1} \partial^{\prime} \hat{\epsilon}_{j}^{n-\lambda}\right) .
\end{aligned}
$$

Comparing these expressions, the coefficient of $\sigma$ in $a_{j k}^{\prime}$ is equal to $(-1)^{\lambda+1}$ times the coefficient of $\sigma^{-1}$ in $a_{k j}$. Therefore

as asserted.

$$
a_{j k}^{\prime}=(-1)^{\lambda+1} \bar{a}_{k j}
$$

Proof of Duality Theorem. First suppose that there exists a nice function $f$ on $W$ such that all critical points have index $\lambda$ or $\lambda+1$. Then the complex $\bar{C}$ associated with $f$ is completely described by the matrix $\left(a_{k j}\right)$ which corresponds to

$$
\bar{\partial}: \bar{C}_{\lambda+1} \rightarrow \bar{C}_{\lambda} \text {. }
$$

Since $\bar{C}$ is acyclic it is clear that $\left(a_{k j}\right)$ is nonsingular, and that the canonical homomorphism

$$
\eta: \mathrm{GL}(\alpha, Z \Pi) \rightarrow \mathrm{Wh}(\Pi)
$$

carries $\left(a_{k j}\right)$ to $(-1)^{\lambda} \tau(W, M)$.

Similarly the dual complex $\bar{C}^{\prime}$ is completely described by the matrix $\left(a_{j \mathrm{k}}^{\prime}\right)$ which corresponds to

$$
\partial^{\prime}: \bar{C}_{n-\lambda}^{\prime} \rightarrow \bar{C}_{n-\lambda-1}^{\prime},
$$

so that $\eta$ carries $\left(a_{j k}^{\prime}\right)$ to $(-1)^{n-\lambda-1} \tau\left(W, M^{\prime}\right)$. But $\left(a_{j k}^{\prime}\right)$ is, up to sign, the conjugate transpose of $\left(a_{k j}\right)$. Therefore

$$
(-1)^{\lambda} \tau(W, M)=(-1)^{n-\lambda-1} \bar{\tau}\left(W, M^{\prime}\right),
$$

which completes the proof in the special case.

If the dimension $n$ is $\geqq 6$ this actually completes the proof, since such special nice functions are constructed as part of the proof of the $s$-Cobordism Theorem. (See also the proof of the Existence Theorem in $\$ 11$.

This simplification is not really necessary however. It is better simply to prove in general that two chain complexes $\bar{C}$ and $\bar{C}^{\prime}$ which are "dual," in the sense that they satisfy the Duality Lemma, will always have torsions satisfying the Duality Theorem. The argument is not too different from that given in Milnor [1962]; and will be left to the reader. 
REMARK. If the manifolds are not orientable then it is necessary to change the Duality Theorem and Lemma as follows. The conjugation operation in $Z$ II must be replaced by the anti-automorphism $a \rightarrow a^{*}$ which satisfies

$$
\begin{aligned}
& \sigma^{*}=\sigma^{-1} \text { if } \sigma \text { is orientation preserving, } \\
& \sigma^{*}=-\sigma^{-1} \text { otherwise. }
\end{aligned}
$$

This induces an automorphism of Wh(II) which is definitely different from conjugation. Here is an example. If $T_{8}$ is cyclic of order 8 with generator $t$, and if $t^{*}=-t^{-1}$, then the fundamental unit

$$
u=2+\left(t+t^{7}\right)-\left(t^{3}+t^{5}\right)-t^{4} \in Z T_{8}
$$

satisfies $\bar{u}=u$, but $u^{*}=u^{-1}$. No power of $u$ is equal to 1 .

The Duality Theorem has an analogue for $R$-torsion which can be proved in essentially the same way. (Compare Milnor [1962].) The following application is particularly striking.

ThEOREM. If $M$ is a closed orientable manifold of even dimension then every $R$-torsion $\Delta_{h}(M)$ which is defined is equal to 1 .

11. The classification of $h$-cobordisms. The following result is due to Stallings $\left[1965_{2}\right]$. Let $M$ be a smooth, compact, connected manifold without boundary of dimension $n-1$.

Existence Theorem 11.1. If the dimension of $M$ is $\geqq 5$, then for every $\tau_{0} \in \mathrm{Wh}\left(\pi_{1} M\right)$ there exists an h-cobordism $\left(W ; M, M^{\prime}\right)$ with $\tau(W, M)=\tau_{0}$.

Here is an outline of the proof. It is convenient to use the language of "attaching handles" (Small [1961]).

Let $\left(a_{i j}\right)$ be a $k \times k$ nonsingular matrix over $Z$ III which represents $\tau_{0}$. Start with the product $M \times[0,1]$ and attach $k$ handles of index +2 to the right hand side. The attaching maps

$$
S^{1} \times D^{n-2} \rightarrow M \times[1]
$$

should be "trivial" so that the resulting manifold $W_{1}$ can also be described as that obtained from $M \times[0,1]$ by pasting $k$ copies of $S^{2} \times D^{n-2}$ along its right hand boundary. The left and right hand boundaries of $W_{1}$ will be denoted by $M$ and $M_{1}$ respectively.

The group $\pi_{2}\left(W_{1}, M\right) \cong H_{2}\left(\hat{W}_{1}, \hat{M}\right)$ is clearly free over $Z\left(\pi_{1} M\right)$ with $k$ preferred generators.

LEMMA 11.2. Each element of $\pi_{2}\left(W_{1}, M\right)$ can be represented by $a$ 2-sphere $S \subset M_{1}$ which is embedded with trivial normal bundle. 
PROOF. Since $\pi_{1} W_{1} \cong \pi_{1} M$ we have

$$
\pi_{2} W_{1} \rightarrow \pi_{2}\left(W_{1}, M\right) \rightarrow 0 .
$$

Furthermore

$$
\pi_{2} M_{1} \rightarrow \pi_{2} W_{1} \rightarrow 0
$$

since $W_{1}$ has a dual handlebody presentation in which it is obtained from $M_{1} \times[0,1]$ by attaching $k$ handles of index $n-2>2$. Therefore the given homotopy class can be represented by an element of $\pi_{2} M_{1}$. Each element of $\pi_{2} M_{1}$ is represented by an imbedded sphere $S$ since the dimension $n-1$ is $\geqq 5$.

We must try to choose $S$ so that its normal bundle is trivial. To verify that the normal bundle of $S$ is trivial it is sufficient to check that the Stiefel-Whitney class $w_{2}$ of $W_{1}$ annihilates the homology class of $S$ in $W_{1}$. But this Stiefel-Whitney class determines a homomorphism

$$
\pi_{2} W_{1} \rightarrow H_{2} W_{1} \rightarrow Z_{2} \text {. }
$$

By the construction of $W_{1}$ it is clear that we can choose elements $\mu_{1}, \cdots, \mu_{k} \in \pi_{2} W_{1}$ which represent a basis for $\pi_{2}\left(W_{1}, M\right)$; and so that $w_{2}$ annihilates the homology class of each of the $\mu_{i}$. Hence, if the homotopy class of $S$ in $W_{1}$ is chosen carefully, the normal bundle of $S$ in $W_{1}$, and in $M_{1}$, will be trivial. This proves the Lemma.

Now the $k$ rows of the matrix $\left(a_{i j}\right)$ represent elements of $\pi_{2}\left(W_{1}, M\right)$ which are represented by spheres appropriately imbedded in $M_{1}$. Using these $k$ 2-spheres to attach handles of index 3 to $W_{1}$, we obtain the required manifold $W$. Let $M^{\prime}$ be the right hand boundary.

Clearly $\pi_{1} M \cong \pi_{1} W_{1} \cong \pi_{1} W$; and $H_{*}(\hat{W}, \hat{M})=0$ since the matrix $\left(a_{i j}\right)$ is nonsingular. Therefore $M$ is a deformation retract of $W$. (Compare Whitehead [1949].) On the other hand $\pi_{1} M^{\prime} \cong \pi_{1} W$ since $W$ can be formed from $M^{\prime} \times[0,1]$ by attaching handles of index $n-3 \geqq 3$ and $n-2 \geqq 4$. Furthermore, using the Duality Lemma of $\$ 10$, we see that $H_{*}\left(\hat{W}, \hat{M}^{\prime}\right)$ is also zero. Therefore $\left(W ; M, M^{\prime}\right)$ is an $h$-cobordism.

Since $\tau(W, M)$ is clearly equal to $\tau_{0}$, this completes the proof.

Combining the Existence Theorem with the $s$-Cobordism Theorem (\$10), we obtain the following.

Uniqueness Theorem 11.3. Let $\left(W ; M, M_{1}\right)$ and $\left(W_{2} ; M, M_{2}\right)$ be two $h$-cobordisms of dimension $\geqq 6$ with the same torsion:

$$
\tau\left(W_{1}, M\right)=\tau\left(W_{2}, M\right)=\tau_{0} .
$$

Then $W_{1}$ is diffeomorphic to $W_{2}$ under a diffeomorphism which preserves $M$. 
Proof. (Compare Figure 8.) Choose an $h$-cobordism $\left(W^{\prime} ; M_{1}, M^{\prime}\right)$ with $\tau\left(W^{\prime}, M_{1}\right)=-\tau_{0}$. (We are identifying the fundamental groups

$$
\pi_{1} M \cong \pi_{1} W_{k} \cong \pi_{1} M_{k}
$$

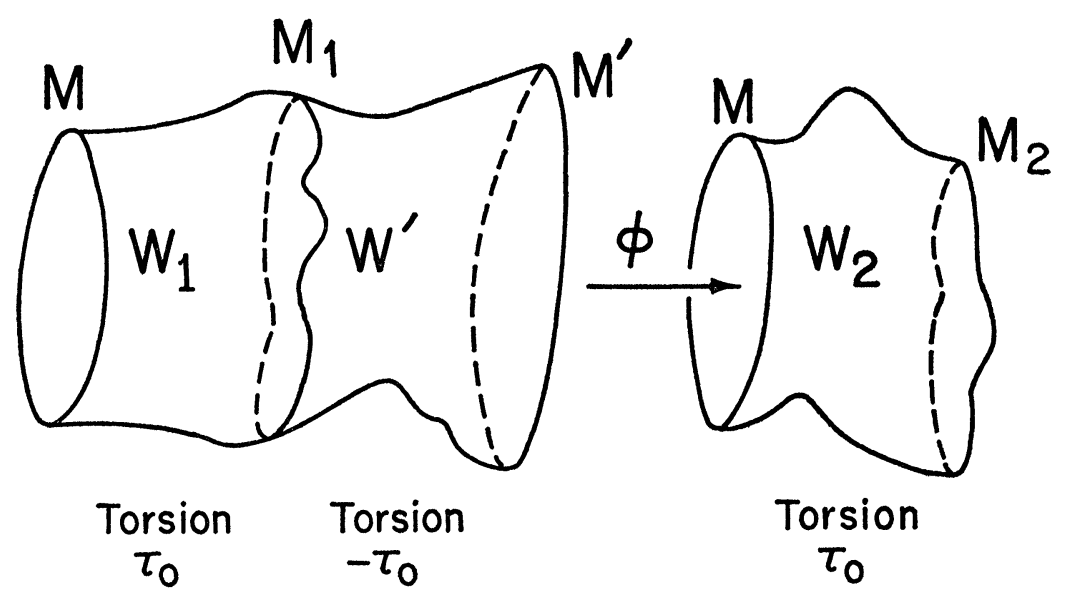

Figure 8 . The composite $h$-cobordism is diffeomorphic both to $W_{1}$ and to $W_{2}$.

in the obvious way.) Let $W_{1} \cup W^{\prime}$ be a smooth manifold obtained by pasting $W_{1}$ and $W^{\prime}$ together along $M_{1}$. Then

$$
\tau\left(W_{1} \cup W^{\prime}, M\right)=\tau\left(W_{1}, M\right)+\tau\left(W_{1} \cup W^{\prime}, W_{1}\right)=\tau_{0}-\tau_{0}=0,
$$

hence $W_{1} \cup W^{\prime} \approx M \times[0,1]$. In particular we obtain a diffeomorphism $\phi: M^{\prime} \rightarrow M$.

Similarly form $W_{1} \cup W^{\prime} \cup_{\phi} W_{2}$. Since $W_{1} \cup W^{\prime}$ is a product it follows that

$$
W_{1} \cup W^{\prime} \cup_{\phi} W_{2} \approx(M \times[0,1]) \cup W_{2} \approx W_{2} .
$$

But a similar argument shows that $W^{\prime} \cup_{\phi} W_{2}$ is a product, hence

$$
W_{1} \cup W \cup_{\phi} W_{2} \approx W_{1} \cup\left(M_{1} \times[0,1]\right) \approx W_{1} .
$$

This completes the proof.

Let us try to test the power of these theorems by trying to classify manifolds which are $h$-cobordant to a given manifold $M_{0}$. We must first ask the following question. Given an $h$-cobordism ( $W ; M_{0}, M^{\prime}$ ) can it happen that $M^{\prime}$ is diffeomorphic to $M_{0}$ even though $W$ is not a product?

Here is a method for constructing examples. Given any $h$-cobordism ( $W ; M_{0}, M_{1}$ ) let $2 W$ denote the "double" of $W$ which is obtained by pasting together two copies of $W$ along the right hand boundary $M_{1}$. 
Then clearly $\left(2 W ; M_{0}, M_{0}^{\prime}\right)$ is an h-cobordism, where $M_{0}$ and $M_{0}^{\prime}$ denote two copies of the same manifold.

Lemma 11.4. The torsion $\tau\left(2 W, M_{0}\right)$ is equal to $\tau\left(W, M_{0}\right)+(-1)^{n-1}$ $\bar{\tau}\left(W, M_{0}\right)$.

The proof is straightforward.

Thus, surprisingly enough, $2 W$ is not always a product. For example if $\Pi$ is finite commutative and the dimension $n \geqq 7$ is odd, then $\tau\left(2 W, M_{0}\right)=2 \tau\left(W, M_{0}\right)$, so $2 W$ is a product only if $W$ is a product.

An interesting consequence is the following.

Theorem 11.5. If $M$ has even dimension and $\pi_{1} M$ is finite, then there are only finitely many distinct manifolds which are h-cobordant to $M$.

In contrast, if $M$ has odd dimension, there may well be infinitely many such manifolds. (Compare \$12.9.)

Proof. Let $G \subset \mathrm{Wh}\left(\pi_{1} M\right)$ denote the subgroup consisting of all elements of the form $\omega+\bar{\omega}$. Since $\mathrm{Wh}\left(\pi_{1} M\right)$ is finitely generated, and since

$$
\bar{\omega} \equiv \omega \quad \text { (modulo elements of finite order) }
$$

by 6.10 , it follows easily that $G$ is a subgroup of finite index.

Consider two $h$-cobordisms $\left(W^{\prime} ; M, M^{\prime}\right)$ and $\left(W^{\prime \prime} ; M, M^{\prime \prime}\right)$ which satisfy

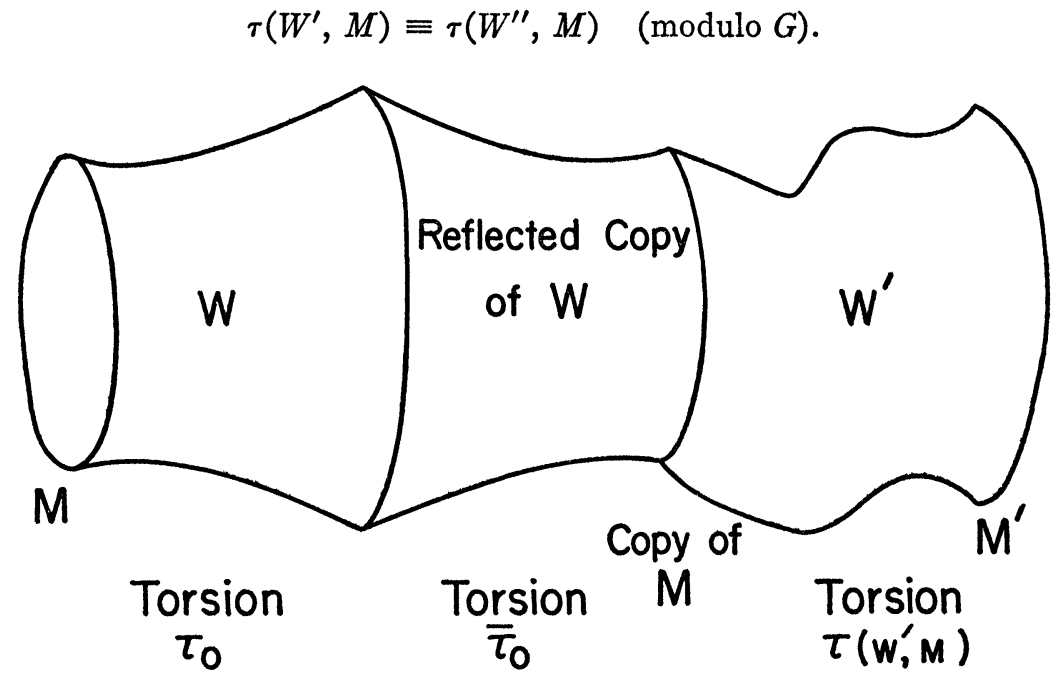

FIGURE 9. The composite $h$-cobordism is diffeomorphic to $W^{\prime \prime}$. 
Setting $\tau\left(W^{\prime \prime}, M\right)=\tau\left(W^{\prime}, M\right)+\tau_{0}+\bar{\tau}_{0}$, construct an $h$-cobordism ( $W ; M, M_{1}$ ) with torsion $\tau_{0}$. Paste the double of $W$ onto $W^{\prime}$ so as to obtain an $h$-cobordism $\left(2 W \subset W^{\prime} ; M, M^{\prime}\right)$ with torsion $\tau_{0}+$ $(-1)^{n-1} \bar{\tau}_{0}+\tau\left(W^{\prime}, M^{\prime}\right)$ equal to $\tau\left(W^{\prime \prime}, M\right)$. Then $2 W \cup W^{\prime} \approx W^{\prime \prime}$ and hence $M^{\prime} \approx M^{\prime \prime}$. Thus the number of distinct manifolds $h$-cobordant to $M$ is at most equal to the order of the quotient group $\mathrm{Wh}(\Pi) / G$. This completes the proof.

12. Lens spaces. The lens spaces of Tietze and de Rham serve as marvellous examples for the study of torsion. First some notation. Let $m \geqq 3$ be a fixed integer and let $\Pi=T_{m}$ be the cyclic group of order $m$ with generator $t$. Let $S^{2 n-1} \subset C^{n}$ be the unit sphere in complex $n$-space, with a standard orientation.

Given primitive $m$ th roots of unity $\alpha_{1}, \cdots, \alpha_{n} \in C$ define an action of $\Pi$ on $S^{2 n-1}$ by the formula

$$
t\left(z_{1}, \cdots, z_{n}\right)=\left(\alpha_{1} z_{1}, \cdots, \alpha_{n} z_{n}\right) .
$$

The quotient manifold $S^{2 n-1} / \Pi$ is then called a lens space.

In order to index these manifolds conveniently we can introduce residue classes $q_{1}, \cdots, q_{n}$ modulo $m$ by the formula

$$
\alpha_{j}=\exp \left(2 \pi i q_{j} / m\right) .
$$

Thus if $\alpha_{0}=\exp (2 \pi i / m)$ is the standard root of unity, then $\alpha_{j}=\alpha_{0}^{q_{j}}$. The "reciprocal" residue classes $r_{1}, \cdots, r_{n}$ are also important. That is, we define $r_{j}$ by the congruence

$$
q_{j} r_{j} \equiv 1 \quad(\bmod m)
$$

so that $\alpha_{0}=\alpha_{j}^{r_{j}}$. Following Franz the notation $L_{m}\left(r_{1}, \cdots, r_{n}\right)$ (or briefly $\left.L\left(r_{1}, \cdots, r_{n}\right)\right)$ will be used for the corresponding lens space $S^{2 n-1} / \Pi$.

Note that $L\left(r_{1}, \cdots, r_{n}\right)$ is a Riemannian manifold with a preferred orientation, and that its fundamental group $\Pi$ has a preferred generator $t$. It will be convenient to say that two lens spaces are isomorphic if they are related by a diffeomorphism which preserves the Riemannian metric and the orientation and the preferred generator.

Some diffeomorphisms. Clearly the order of the numbers $r_{1}, \cdots, r_{n}$ is not important. Thus if $\left(r_{1}^{\prime}, \cdots, r_{n}^{\prime}\right)$ is a permutation of $\left(r_{1}, \cdots, r_{n}\right)$ then $L\left(r_{1}^{\prime}, \cdots, r_{n}^{\prime}\right)$ is isomorphic to $L\left(r_{1}, \cdots, r_{n}\right)$. If we change the sign of one of the $r_{k}$ the only effect is to reverse the orientation. Hence every lens space is isomorphic to one of the form $\pm L\left(r_{1}, \cdots, r_{n}\right)$ with

$$
1 \leqq r_{1} \leqq r_{2} \leqq \cdots \leqq r_{n}<m / 2 .
$$


(Here $-L$ denotes $L$ with orientation reversed.) It will turn out later that every lens space is isomorphic to one and only one lens space of this form. As an example, if $m$ is equal to 3, 4, or 6 then every lens space is isomorphic to $\pm L(1, \cdots, 1)$.

If the preferred generator for $\Pi$ is altered, the effect is to replace $\left(r_{1}, \cdots, r_{n}\right)$ by some constant multiple $\left(k r_{1}, \cdots, k r_{n}\right)$. Hence if we do not wish to distinguish any particular generator for $\Pi$, the notation $L\left(r_{1}: r_{2}: \cdots: r_{n}\right)$ is appropriate.

Metric properties. As Riemannian manifolds the lens spaces of a given dimension are all locally isometric. But they are clearly distinguished by global properties. For example the space $L(1: 1: \cdots: 1)$ of dimension $2 n-1$ possesses a transitive $n^{2}$-dimensional group of isometries: namely the full unitary group $U(n)$. In contrast, if the residue classes $\pm r_{1}, \cdots, \pm r_{n}$ are all distinct, then the group of isometries of $L\left(r_{1}, \cdots, r_{n}\right)$ is only the $n$-dimensional group $U(1) \times \cdots \times U(1)$.

12.1. Homotopy type. According to Rueff [1938] and Olum [1953]: there exists a homotopy equivalence

$$
L\left(r_{1}^{\prime}, \cdots, r_{n}^{\prime}\right) \rightarrow L\left(r_{1}, \cdots, r_{n}\right)
$$

which preserves the orientation and preferred generator if and only if

$$
r_{1}^{\prime} \cdots r_{n}^{\prime} \equiv r_{1} \cdots r_{n} \quad(\bmod m) .
$$

Hence the necessary and sufficient condition for the existence of a homotopy equivalence which is not required to preserve orientation and generator is that

$$
r_{1}^{\prime} \cdots r_{n}^{\prime} \equiv \pm k^{n} r_{1} \cdots r_{n} \quad(\bmod m)
$$

for some integer $k$.

12.2. Tangent bundle. The preferred generator $t$ for $\Pi$ determines a preferred generator $x$ for the dual group

$$
H^{2}(L ; \boldsymbol{Z}) \cong \operatorname{Ext}(\Pi, \boldsymbol{Z}) \cong \operatorname{Hom}(\Pi, \boldsymbol{R} / \boldsymbol{Z}) \text {. }
$$

The total Pontrjagin class $p=1+p_{1}+p_{2}+\cdots$ of the lens space $L\left(r_{1}, \cdots, r_{n}\right)$ turns out to be given by the formula

$$
p=\left(1+q_{1}^{2} x^{2}\right)\left(1+q_{2}^{2} x^{2}\right) \cdots\left(1+q_{n}^{2} x^{2}\right)
$$

(where $q_{i} r_{i} \equiv 1$ ). Thus the individual Pontrjagin classes are the elementary functions of the $q_{i}^{2} x^{2}$ :

and so on.

$$
\begin{aligned}
& p_{1}=\left(q_{1}^{2}+\cdots+q_{n}^{2}\right) x^{2}, \\
& p_{2}=\left(q_{1}^{2} q_{2}^{2}+q_{1}^{2} q_{3}^{2}+\cdots+q_{n-1}^{2} q_{n}^{2}\right) x^{4},
\end{aligned}
$$


Example. A lens space can be parallelizable only if its dimension is 3 , or its dimension is 7 and

$$
q_{1}^{2}+q_{2}^{2}+q_{3}^{2}+q_{4}^{2} \equiv 0 \quad(\bmod m) .
$$

(For some values of $m$, e.g., if $m$ is a multiple of 3 or 8 , this congruence cannot be satisfied.)

I will not try to give any details here. A complete determination of the stable tangent bundle of a lens space has been given by Szczarba [1964] and Folkman [1965]. In fact Folkman has proved the following remarkable result: If $m$ is prime, then a high dimensional lens space $L_{m}$ is determined up to isomorphism by its homotopy type and its stable tangent bundle.

12.3. Cell structure. Each lens space $L$ can be given the structure of a CW-complex with one cell in each dimension. The boundary relations in $\hat{L}=S^{2 n-1}$ then take the form

$$
\begin{aligned}
\partial \hat{e}^{2 k-1} & =\left(t^{r k}-1\right) \hat{e}^{2 k-2} \quad \text { for } 1 \leqq k \leqq n, \\
\partial \hat{e}^{2 k} & =\left(1+t+t^{2}+\cdots+t^{m-1}\right) \hat{e}^{2 k-1} \quad \text { for } 1 \leqq k<n .
\end{aligned}
$$

(Compare Franz [1935] and de Rham [1950].) This CW-complex has a subdivision which is a $C^{1}$-triangulation of $L$. (See Milnor [1961], as well as Franz and de Rham.) Hence any torsion invariants which can be computed from the cell structure will be diffeomorphism invariants.

The $R$-torsion invariants can readily be computed, and can be used to classify lens spaces. Instead we will introduce an alternative form of torsion which is somewhat more convenient to work with.

Definition. A finite complex or a compact smooth manifold will be called special if the fundamental group $\Pi$ is finite abelian and operates trivially on the rational homology groups of the universal covering space.

For such special complexes we will define a torsion invariant in the rational group ring $Q \Pi$.

For any finite group $\Pi$ let $N \subset Q \Pi$ denote the kernel of the canonical homomorphism $\boldsymbol{Q} \Pi \rightarrow \boldsymbol{Q}$ which caries all group elements to +1 . Let $\Sigma \in Z \Pi$ denote the sum of all of the group elements. Then clearly the algebra splits as the direct sum

$$
\mathbf{Q} \Pi=N \oplus(\Sigma)
$$

of two mutually annihilating two sided ideals (where $(\Sigma)=Q \Sigma$ ).

Note that $N$ itself can be thought of as an algebra which is isomorphic to the $Q \Pi /(\Sigma)$. (The identity element of $N$ is equal to $1-\Sigma / m$, where $m$ is the order of $\Pi$.) 
Let $X$ be a finite complex with fundamental group $\Pi$. The direct sum decomposition of $Q \Pi$ gives rise to corresponding decompositions of chain and homology modules

$$
\begin{aligned}
& C(\hat{X} ; Q)=N C(\hat{X} ; Q) \oplus \Sigma C(\hat{X} ; Q), \\
& H(\hat{X} ; Q)=N H(\hat{X} ; Q) \oplus \Sigma H(\hat{X} ; Q) .
\end{aligned}
$$

Now if $\Pi$ operates trivially on $H(\hat{X} ; \boldsymbol{Q})$ then clearly $N H(\hat{X} ; \boldsymbol{Q})=0$, so that the complex $N C(\hat{X} ; \boldsymbol{Q})$ is acyclic.

Clearly $N C(\hat{X} ; Q)$ is $N$-free, with a preferred basis which corresponds to the preferred basis for $C(\hat{X} ; \boldsymbol{Z})$ under the natural homomorphism

$$
C(\hat{X} ; Z) \rightarrow C(\hat{X} ; Q) / \Sigma C(\hat{X} ; Q)=N C(\hat{X} ; Q) .
$$

Hence the torsion

$$
\tau(N C(\hat{X} ; Q)) \in \bar{K}_{1} N /(\text { image } \Pi)
$$

is well defined. As in $\S 7$, this torsion is invariant under subdivision.

Now suppose that $\Pi$ is abelian. Then $N$ is isomorphic to a cartesian product of (cyclotomic) fields, hence $K_{1} N$ can be identified with the group $U(N)$ of units of $N$. We will switch to a multiplicative notation, and write $\Delta(X) \in U(N)$ for an element corresponding to the torsion $\tau(N C(\hat{X} ; Q))$. Thus we have proved:

Lemma 12.4. To each special complex $X$ there corresponds the torsion

$$
\Delta(X) \in U(N) \subset N \subset Q \Pi
$$

which is well defined up to multiplication by plus or minus a group element. This torsion is invariant under subdivision of $X$.

It will be convenient to use the notation $\Delta \sim \Delta^{\prime}$ to mean that

$$
\Delta= \pm \sigma \Delta^{\prime}, \text { for some } \sigma \in \Pi \text {. }
$$

The strength of this torsion invariant is shown by the following.

Lemma 12.5. A homotopy equivalence $f: X \rightarrow Y$ between special complexes is a simple homotopy equivalence (that is $\tau(f)=0$ ) if and only if $f_{*} \Delta(X) \sim \Delta(Y)$.

Proof. This follows easily from $\$ 8.1$.

Now let us look at a lens space $L=L\left(r_{1}, \cdots, r_{n}\right)$. This is clearly a "special" manifold. Modulo the ideal $(\Sigma)$ the boundary relations 12.3 take the form 


$$
\begin{aligned}
\partial \hat{e}^{2 k-1} & =\left(t^{r k}-1\right) \hat{e}^{2 k-2}, \\
\partial \hat{e}^{2 k} & =0 .
\end{aligned}
$$

Since we know that $N C(\hat{L}: Q)$ is acyclic it follows that each $\left(t^{r}-1\right)$ must be a unit of $N$. (Alternatively the identity

$$
(t-1)\left(1+2 t+3 t^{2}+\cdots+m t^{m-1}\right)=m-\Sigma
$$

shows directly that $t-1$ is a unit modulo $(\Sigma)$.) Clearly the torsion is given by

$$
\Delta(L) \sim\left(t^{r_{1}}-1\right)\left(t^{r_{2}}-1\right) \cdots\left(t^{r_{n}}-1\right) .
$$

(This turns out to be an expression with integer coefficients, although a priori we could have expected rational coefficients.)

In order to classify lens spaces we must know what relations exist between the $\phi(m)$ elements $t^{r}-1$ of $U(N)$. The identity

$$
\left(t^{r}-1\right)=-t^{r}\left(t^{-r}-1\right)
$$

shows that $\left(t^{r}-1\right) \sim\left(t^{-r}-1\right)$.

12.6. Franz IndePendence Lemma. The $\phi(m) / 2$ units $t^{r}-1$ $\in U(N)$, where

$$
1 \leqq r<m / 2, \quad(r, m)=1,
$$

do not satisfy any multiplicative relations.

Since the proof is rather difficult, we will simply refer to Franz [1935] or de Rham [1964 $]$. (Actually Franz shows only that there can be no relation of the form $\prod\left(t^{r}-1\right)^{a_{r}} \sim 1$ where the exponents $a_{r}$ add up to zero. But the possibility of such a relation in which $\sum a_{r} \neq 0$ is easily ruled out. Compare Lemma 12.10 below.)

Classification Theorem 12.7. If two lens spaces have the same torsion invariant $\Delta \in U(N) / \pm \Pi$ then they are isomorphic, up to orientation. Each lens space is isomorphic to a unique lens space of the form $\pm L\left(r_{1}, \cdots, r_{n}\right)$ with $1 \leqq r_{1} \leqq \cdots \leqq r_{n}<m / 2$.

In particular it follows that two lens spaces which are diffeomorphic must actually be isometric as Riemannian manifolds. (Compare §8.2.)

Proof. This follows immediately from the Franz lemma.

Now let us study an $h$-cobordism $\left(W ; M, M^{\prime}\right)$ between two "special" manifolds. All three fundamental groups will be denoted by $\Pi$.

THEOREM 12.8. Suppose that there exists an h-cobordism between the odd dimensional special manifolds $M, M^{\prime}$ which is compatible with a given identification of fundamental groups. Then

$$
\Delta\left(M^{\prime}\right) \sim u^{2} \Delta(M)
$$


for some unit $u$ of $Z \Pi$, where $u^{2} \sim 1$ if and only if $\tau(W, M)=0$. Conversely, if the dimension is $\geqq 5$, then given $M$ and $u$ one can construct $a$ corresponding $h$-cobordism.

Proof. Given the $h$-cobordism $\left(W ; M, M^{\prime}\right)$ we have

$$
\begin{aligned}
\Delta(W) & \sim \Delta(W, M) \Delta(M) \\
& \sim \Delta\left(W, M^{\prime}\right) \Delta\left(M^{\prime}\right) .
\end{aligned}
$$

Since $M$ and $M^{\prime}$ are deformation retracts, the Whitehead torsions $\tau(W, M)$ and $\tau\left(W, M^{\prime}\right)$ are defined. Furthermore

$$
\tau(W, M)=-\bar{\tau}\left(W, M^{\prime}\right)
$$

by $\$ 10$. Switching to the multiplicative notation, let $u$ and $\bar{u}^{-1}$ denote the units of $Z \Pi$ which correspond to these two torsion invariants. Clearly the homomorphism

$$
Z \Pi \rightarrow Q \Pi /(\Sigma) \cong N
$$

carries $u$ to $\Delta(W, M)$. Therefore

$$
\Delta(W) \sim u \Delta(M) \sim \bar{u}^{-1} \Delta\left(M^{\prime}\right)
$$

hence

$$
\Delta\left(M^{\prime}\right) \sim u \bar{u} \Delta(M) .
$$

Since $u \sim \bar{u}$ by $\S 6.7$ this proves the first statement.

If $u^{2} \sim 1$ then clearly $2 \tau(W, M)=0$. Since $\mathrm{Wh}(\Pi)$ is torsion free, this implies that $\tau(W, M)=0$. (In dimensions $\geqq 5$ it implies that $W \approx M \times[0,1]$.)

Since the final statement of Theorem 12.8 follows immediately from $\$ 11$, this completes the proof.

Corollary 12.9. Let $M$ be a special manifold of dimension $2 n-1$ $\geqq 5$. If $Z \Pi$ has a nontrivial unit then there exist infinitely many distinct manifolds in the h-cobordism class of $M$. On the other hand if ZII has only trivial units then every h-cobordism $\left(W ; M, M^{\prime}\right)$ is a product.

The proof of 12.9 is immediate. (Compare Milnor [1964].)

REMARK. According to Higman the ring $Z \Pi$ has a nontrivial unit unless $\Pi$ has exponent $2,3,4$, or 6 . (See $\$ 6.5$.) If $\Pi$ does have exponent $2,3,4$, or 6 then of course $\mathrm{Wh}(\Pi)=0$.

To conclude the discussion of special manifolds, here are some further results whose proofs will be left as exercises for the reader.

Assertion 1. A homotopy equivalence between even dimensional special manifolds is always a simple homotopy equivalence. 
(I do not know whether $\Delta(M) \sim 1$ for every even dimensional special manifold.)

Assertion 2. If $X$ and $Y$ are special complexes then $\Delta(X \times Y) \sim 1$. (Compare Kwun and Szczarba [1965].)

Assertion 3. If $M$ is an odd dimensional closed orientable manifold and if the $R$-torsion $\Delta_{h}(M)$ is defined for some representation $h$ such that

$$
h_{*}: \mathrm{Wh}(\Pi) \rightarrow R^{+}
$$

is nontrivial, then there are infinitely many distinct manifolds in the $h$-cobordism class of $M$. ("Distinct" in the sense that no two are related by a diffeomorphism which is compatible with the given identification of fundamental groups.)

Theorem 12.8 suggests the importance of finding out precisely what units exist within the ring $Z \Pi$. Here is a preliminary estimate when $\Pi$ is cyclic of order $m>2$. Let $r_{1}, \cdots, r_{n}$ and $s_{1}, \cdots, s_{p}$ be prime to $m$.

Lemma 12.10. There exists a unit $u$ of $Z \mathrm{ZI}$ satisfying the equation

$$
\left(t^{r_{1}}-1\right) \cdots\left(t^{r_{n}}-1\right)=u\left(t^{s_{1}}-1\right) \cdots\left(t^{s_{p}}-1\right)
$$

if and only if $n=p$ and

$$
r_{1} \cdots r_{n} \equiv \pm s_{1} \cdots s_{n}(\bmod m) .
$$

This unit $u$ is uniquely determined, if it exists. Furthermore the canonical homomorphism $\boldsymbol{Z} \Pi \rightarrow \boldsymbol{Z}$ carries $u$ to $u(1)= \pm 1$; where

$$
u(1) \equiv r_{1} \cdots r_{n} / s_{1} \cdots s_{n} \quad(\bmod m) .
$$

(Compare the homotopy classification theorem 12.1.)

Proof. First suppose that $u$ exists and that $n=p$. Let $r_{i} \equiv s_{i} x_{i}$ $(\bmod m)$ with $x_{i}>0$. Since each $\left(t^{s_{i}}-1\right)$ is a unit modulo $\Sigma$ we can divide to obtain

$$
u \equiv \coprod_{i=1}^{n}\left(1+t^{s_{i}}+t^{2 s_{i}}+\cdots+t^{\left(x_{i}-1\right) s_{i}}\right) \quad(\bmod \Sigma) ;
$$

or, more precisely,

$$
u \equiv \amalg\left(1+t^{s_{i}}+\cdots+t^{\left(x_{i}-1\right) s_{i}}\right)+k \Sigma
$$

for some integer $k$. Mapping $t$ into 1 this equality in $Z \Pi$ transforms in to the equality

$$
u(1)=x_{1} \cdots x_{n}+k m
$$

in $Z$. This proves that 


$$
u(1) \equiv x_{1} \cdots x_{n} \equiv r_{1} \cdots r_{n} / s_{1} \cdots s_{n}(\bmod m)
$$

where $u(1)$ clearly must be \pm 1 . Furthermore, since $x_{1}, \cdots, x_{n}$ are given, and $m>2$, the equality (2) can be solved uniquely for $k$. Plugging this value of $k$ into (1) we have shown that $u$ is uniquely determined.

Conversely suppose that we are given $r_{1}, \cdots, r_{n}$ and $s_{1}, \cdots, s_{n}$ satisfying the required congruence. The above argument shows that there exist unique elements $u$ and $v$ of $Z \Pi$ satisfying the conditions

$$
\begin{aligned}
& \left(t^{r_{1}}-1\right) \cdots\left(t^{r_{n}}-1\right)=u\left(t^{s_{1}}-1\right) \cdots\left(t^{s_{n}}-1\right), \\
& \left(t^{s_{1}}-1\right) \cdots\left(t^{s_{n}}-1\right)=v\left(t^{r_{1}}-1\right) \cdots\left(t^{r_{n}}-1\right)
\end{aligned}
$$

and

$$
u(1)=v(1)= \pm 1 \text {. }
$$

Multiplying the first equation by $v$ this implies that

$$
\left(t^{s 1}-1\right) \cdots\left(t^{s n}-1\right)=u v\left(t^{s 1}-1\right) \cdots\left(t^{s n}-1\right) .
$$

But, by the uniqueness statement, this is only possible if $u v=1$. Therefore $u$ is a unit.

To complete the proof we need only exclude the case $n \neq p$. But if, say, $n<p$, then dividing as above we would obtain

$$
\coprod_{1}^{n}\left(1+t^{s_{i}}+\cdots+t^{\left(x_{i}-1\right) s_{i}}\right) \equiv u \coprod_{n+1}^{p}\left(t^{s_{i}}-1\right) \quad(\bmod \Sigma)
$$

Mapping $t$ into 1 this would imply

$$
x_{1} \cdots x_{n} \equiv \pm 0 \quad(\bmod m),
$$

which is impossible.

REMARK. Bass [to appear] has shown that the units described by Lemma 12.10 generate a subgroup of at most finite index in $\mathrm{Wh}(\mathrm{II})$.

Now let us look at the question of cobordism between lens spaces. Atiyah and Bott (unpublished) have obtained an extraordinary new fixed point formula which can be used to prove the following. Let $G$ be a compact group which operates smoothly on a sphere or homology sphere $\Sigma^{n}$ with two fixed points $p$ and $q$, the action being free except at $p$ and $q$.

THEOREM 12.11. If these hypotheses are satisfied, then the action of $G$ on the tangent space of $\Sigma^{n}$ at $p$ is equivalent to the action at $q$.

In other words the group representation $G \rightarrow \mathrm{GL}(n, R)$ obtained from the action at $p$ is equivalent to that obtained from the action at $q$. 
Part of the proof of 12.11 is based on the Franz lemma 12.6. However the main part of the argument involves the Atiyah-Bott fixed point formula for elliptic operators. No details will be given (although we will give the proof of a substantially weaker result in \$12.15).

CoROllary 12.12. If two lens spaces are related by an h-cobordism which is compatible with the preferred generators and orientations, then they are actually isomorphic.

Proof. Let $\left(W ; L_{1}, L_{2}\right)$ be an $h$-cobordism between lens spaces of dimension $2 n-1$. Then the universal covering manifold $\hat{W}$ provides an $h$-cobordism between the spheres $\hat{L}_{1}$ and $\hat{L}_{2}$. The group $\pi_{1} L_{1} \cong \pi_{1} W$ $\cong \pi_{1} L_{2}=\Pi$ of covering transformations operates orthogonally on these two spheres. Hence we can attach one $2 n$-disk to $\hat{W}$ along $\hat{L}_{1}$ and another along $\hat{L}_{2}$ and extend the action of $\Pi$, by letting $\Pi$ operate orthogonally on the disks $D_{1}^{2 n}$ and $D_{2}^{2 n}$. The union $\hat{W} \cup D_{1}^{2 n} \cup D_{2}^{2 n}$ can be given a compatible smoothness structure; and is clearly a homology sphere. Applying the Atiyah-Bott theorem 12.11 we see that the orthogonal action of $\Pi$ on $D_{1}^{2 n}$ is equivalent to the action on $D_{2}^{2 n}$; and hence that $L_{1}$ is equivalent to $\pm L_{2}$.

In fact the sign must be + . For otherwise there would exist a homotopy equivalence $L_{1} \rightarrow-L_{1}$ preserving orientation and preferred generator. But this is impossible by 12.1 .

Corollary 12.13. Any h-cobordism $\left(W ; L, L^{\prime}\right)$ between lens spaces must have trivial torsion. Hence (if the dimension is $\geqq 5$ ) the manifold $W$ must be diffeomorphic to $L \times[0,1]$.

Proof. This follows from 12.12 and 12.8 .

It is essential for these results that $L$ and $L^{\prime}$ should be precisely lens spaces. Here is an example of an $h$-cobordism $(W ; L, M)$ with $\tau(W, L) \neq 0$, where $M$ is a manifold which is indistinguishable from a certain lens space $L^{\prime}$ by all of the more obvious tests.

EXAMPLE 12.14. Let $L=L_{35}(1,1,1,1,1)$ and $L^{\prime}=L_{35}(1,6,6,6,6)$. These nine dimensional lens spaces with $\pi_{1} \cong T_{35}$ are not $h$-cobordant to each other. Nevertheless $L$ is $h$-cobordant to a manifold $M$ which has the same simple homotopy type and the same tangent bundle as $L^{\prime}$.

Proof. By 12.10 , since $6^{2} \equiv 1 \bmod 35$, there exists a unit $u$ of $Z \Pi$ so that

$$
\left(t^{6}-1\right)^{2}=u(t-1)^{2} .
$$

By 12.8 there is an $h$-cobordism $(W ; L, M)$ starting from $L$, and having 


$$
\begin{aligned}
\Delta(M) & \sim u^{2} \Delta(L)=u^{2}(t-1)^{5} \\
& =\left(t^{6}-1\right)^{4}(t-1) \sim \Delta\left(L^{\prime}\right) .
\end{aligned}
$$

According to 12.1 there does exist a homotopy equivalence

$$
f: M \rightarrow L^{\prime} \text {. }
$$

Furthermore it follows from 12.5 that $f$ is a simple homotopy equivalence. We will prove that the induced bundle

$$
f^{*} \text { (tangent bundle of } L^{\prime} \text { ) }
$$

is isomorphic to the tangent bundle of $M$. The obstructions to the existence of such an isomorphism lie in the cohomology groups

$$
H^{k}\left(M ; \pi_{k-1}\left(\mathrm{SO}_{q}\right)\right) \text {. }
$$

Thus there are obstructions in dimensions 4,8 (namely the differences of Pontrjagin classes) and in dimension 9. It follows from 12.2 that the Pontrjagin classes do not distinguish $M$ from $L^{\prime}$. But the 9-dimensional obstruction is an element of order 2 , and hence is not destroyed if we pass to the 35-fold covering space. It follows easily that all of the obstructions are zero; which completes the proof of 12.14 .

Thus 12.12 and 12.13 definitely cannot be proved, using only the methods of the present paper. Nevertheless we will persevere, and see how far it is possible to get making use only of torsions and tangent bundles.

Let $\left(W ; L, L^{\prime}\right)$ be an $h$-cobordism between two lens spaces with fundamental group of order $m$.

THEOREM 12.15. If $m$ is prime and less than 163, then the torsion $\tau(W, L)$ is zero. More generally the same result will be true if $L$ and $L^{\prime}$ are replaced by any manifolds having the simple homotopy types and the same Pontrjagin classes as suitable lens spaces. ${ }^{10}$

The proof of 12.15 will occupy the rest of $\$ 12$.

REMARK. The present proof works for many larger primes, but it definitely breaks down for the case $m=163$.

The following holds without restriction on $m$.

LemMA 12.16. If the indices $r_{i}$ are equal in pairs, say $L=L\left(r_{1}, r_{1}\right.$, $\left.r_{2}, r_{2}, \cdots, r_{k}, r_{k}\right)$ with $n=2 k$ and correspondingly $L^{\prime}=L\left(s_{1}, s_{1}, s_{2}\right.$, $\left.s_{2}, \cdots, s_{k}, s_{k}\right)$, then there cannot exist an orientation reversing $h$ cobordism from $L$ to $L^{\prime}$.

10 Similar generalizations apply throughout the remainder of $\$ 12$. 
COROLLARY. There cannot exist an orientation reversing $h$-cobordism from $L\left(r_{1}: r_{1}: r_{2}: r_{2}: \cdots: r_{k}: r_{k}\right)$ to itself.

(In contrast if $n$ is odd then every lens space $L\left(r_{1}: \cdots: r_{n}\right)$ admits an orientation reversing diffeomorphism. Similarly, if $r^{2} \equiv-1(\bmod$ $m$ ), then the 3 -dimensional lens space

$$
L(1: r) \approx L\left(r: r^{2}\right) \approx L(-1: r)
$$

admits an orientation reversing diffeomorphism.)

Proof of 12.16. For an orientation reversing $h$-cobordism, the homotopy type classification theorem implies that

$$
r_{1} r_{1} \cdots r_{k} r_{k} \equiv-s_{1} s_{1} \cdots s_{k} s_{k} \quad(\bmod m) .
$$

In particular, -1 must be a quadratic residue modulo $m$.

On the other hand for an $h$-cobordism we must have

$$
\left(t^{r_{1}}-1\right)^{2} \cdots\left(t^{r k}-1\right)^{2}= \pm t^{a} u^{2}\left(t^{s_{1}}-1\right)^{2} \cdots\left(t^{s k}-1\right)^{2}
$$

by 12.8 . And by 12.10 the sign which occurs here must also be a minus sign. We will show that this is impossible.

Note that $m$ must have an odd prime factor. For otherwise $m$ would be divisible by 4 ; and hence -1 would not be a quadratic residue. Map $Z \Pi$ into a cyclotomic number field by mapping $t$ into an odd root of unity $\xi$. Then

$$
\left(\xi^{r_{1}}-1\right)^{2} \cdots\left(\xi^{r k}-1\right)^{2}=-\xi^{a} u(\xi)^{2}\left(\xi^{\xi_{1}}-1\right)^{2} \cdots\left(\xi^{s_{k}}-1\right)^{2} .
$$

Thus $-\xi^{a}$ can be expressed as a square within the field $Q[\xi]$; and hence -1 can be expressed as a square. But in an odd cyclotomic field this is impossible, q.e.d.

The proof of Theorem 12.15 will depend on some results from algebraic number theory. Let $\xi$ denote a primitive $m$ th root of unity, with $m=2 d+1$ prime. Let $U$ denote the group of units in the ring $\boldsymbol{Z}[\xi]$ of cyclotomic integers. Then it is known classically that

(1) $U$ is a finitely generated group of rank $d-1$.

(2) The only elements of finite order in $U$ are the roots of unity $\pm \xi^{k}$

(3) The "cyclotomic units" $\left(\xi^{r}-1\right) /(\xi-1) \in U$, with $1<r \leqq d$, satisfy no multiplicative relations. (Compare Kummer [1851], or the Independence Lemma 12.6.) Hence they generate a subgroup of finite index in $U$.

Let $U_{0}$ denote the subgroup generated by the cyclotomic units together with $\pm \xi$. The index of $U_{0}$ in $U$ will be denoted by $h_{2}(m)$. In the customary terminology $h_{2}(m)$ is called the "second factor of the class number" for the cyclotomic field $Q[\xi]$. 
Lemma 12.17 (Kummer.) For all primes $m$ less than 163 the index $h_{2}(m)$ is an odd integer. However $h_{2}(163)$ is even.

Proof. Kummer showed in [1851] that the number of ideal classes in the ring $\boldsymbol{Z}[\xi]$ can be expressed as a product $h_{1} h_{2}$ of integers, where $h_{2}$ is equal to the index $\left[U: U_{0}\right]$ as just described, ${ }^{11}$ and where $h_{1}$ is given by an explicit numerical formula. He tabulated the values of $h_{1}(m)$ for $m<100$. A more extensive tabulation, for $m \leqq 257$, has been given by Schrutka v. Rechtenstamm [1964]. Within this range, and for $m$ prime, only

$$
\begin{aligned}
& h_{1}(29)=8 \\
& h_{1}(113)=1,612,072,001,362,952, \\
& h_{1}(163)=2,708,534,744,692,077,051,875,131,636
\end{aligned}
$$

and

$$
h_{1}(197), \quad h_{1}(239)
$$

are even. ${ }^{12}$

In [1870] Kummer proved that the second factor $h_{2}(m)$ can be even only if $h_{1}(m)$ is even. He made a particular study of the cases $m=29,113$ and 163; showing that $h_{2}(29)$ and $h_{2}(113)$ are odd, but that $h_{2}(163)$ is even. (In fact, he gave an explicit example of an element of order 2 in $U / U_{0}$ for $m=163$.) This completes the proof of 12.17.

REMARK 12.18. The numbers $h_{2}(m)$ seem extraordinarily difficult to compute. However it is known that

$$
h_{2}(m)=h_{1}(m)=1
$$

for $m \leqq 19$. (See Minkowski [1891, p. 296] as well as Wolfskehl [1886] and Kummer [1851, p. 472].) It has been conjectured that $h_{2}(m)=1$ for all $m<97$. (See Schrutka v. Rechtenstamm [1964, p. 4].) For further information see Hasse [1952], and Vandiver and Wahlin [1928].

Now consider the group ring $Z I I$ where $\Pi$ is cyclic of prime order $m=2 d+1$.

Lemma 12.19. Suppose that $h_{2}(m)$ is odd. If the equality

$$
\left(t^{r_{1}}-1\right) \cdots\left(t^{r_{n}}-1\right)= \pm t^{a} u^{2}\left(t^{s_{1}}-1\right) \cdots\left(t^{s_{n}}-1\right)
$$

${ }^{11}$ Alternatively $h_{2}$ is equal to the number of ideal classes in the real subring $z[\xi+\bar{\xi}]$ of the cyclotomic field. For a modern account see Hasse [1952 ].

12 The last two numbers are very roughly equal to $6 \times 10^{36}$ and $2 \times 10^{49}$ respectively. They are divisible by $2^{3}$ and $2^{6}$ respectively. 
holds for some unit $u$ of $\mathrm{ZII}$, where

$$
1 \leqq r_{1} \leqq \cdots \leqq r_{n} \leqq d, \quad 1 \leqq s_{1} \leqq \cdots \leqq s_{n} \leqq d
$$

then the $2 n$ numbers $r_{1}, \cdots, r_{n}, s_{1}, \cdots, s_{n}$ must be equal in pairs. Furthermore the congruence

$$
r_{1} \cdots r_{n} \equiv s_{1} \cdots s_{n} \quad(\bmod m)
$$

must be satisfied.

Proof. Mapping $t$ into $\xi$ we obtain a corresponding equality involving a unit $u(\xi)$ of $\boldsymbol{Z}[\xi]$. Clearly $u(\xi)^{2}$ belongs to the subgroup $U_{0} \subset U$ generated by cyclotomic units. Since the order $h_{2}(m)$ of $U / U_{0}$ is odd, this implies that $u(\xi)$ itself belongs to $U_{0}$. The statement that the numbers $r_{1}, \cdots, r_{n}, s_{1}, \cdots, s_{n}$ are equal in pairs now follows by inspection, making use of 12.6 .

Now the proof of 12.16 can be used to show that the plus sign must hold in Equation (1). In other words the congruence $r_{1} \cdots r_{n} \equiv$ $+s_{1} \cdots s_{n}$ must be satisfied. This proves Lemma 12.19.

EXAMPLE. If $n=2$ then the statement that the numbers $r_{1}, r_{2}, s_{1}, s_{2}$ are "equal in pairs" means that either

$$
r_{1}=s_{1}, \quad r_{2}=s_{2}
$$

or

$$
r_{1}=r_{2}, \quad s_{1}=s_{2} .
$$

(The third possibility: $r_{1}=s_{2}, r_{2}=s_{1}$ may be ignored because of our assumption that $r_{1} \leqq r_{2}, s_{1} \leqq s_{2}$.) But if $r_{1}=r_{2}, s_{1}=s_{2}$ then the congruence $r_{1} r_{2} \equiv s_{1} s_{2}$ implies (since $m$ is prime) that $r_{1} \equiv \pm s_{1}$ and hence that $r_{1}=s_{1}$. Thus in any case we must have

$$
r_{1}=s_{1}, \quad r_{2}=s_{2}
$$

so that $L\left(r_{1}, r_{2}\right)=L\left(s_{1}, s_{2}\right)$. This proves Theorem 12.15 for the case $n=2$.

Proof of 12.15 For $n>2$. Setting the total Pontrjagin class of $L$ equal to the total Pontrjagin class of $L^{\prime}$ we obtain

(3) $\left(1+x^{2} r_{1}^{-2}\right) \cdots\left(1+x^{2} r_{n}^{-2}\right)=\left(1+x^{2} s_{1}^{-2}\right) \cdots\left(1+x^{2} s_{n}^{-2}\right)$

where $x^{k} \in H^{2 k}(L ; \boldsymbol{Z})$ is an element of order $m$ for $k<n$, and is zero for $k \geqq n$.

The following result will be proved by induction on $n$.

Lemma 12.20. If Equations (1), (2) and (3) hold, and if $h_{2}(m)$ is odd, then $r_{1}=s_{1}, \cdots, r_{n}=s_{n}$. 
The proof will be divided into two cases. It follows immediately from Lemma 12.19 that these two cases exhaust all of the possibilities.

Case 1. Suppose that some $r_{i}$ is equal to some $s_{j}$. Then dividing Equation (1) by the equality $\left(t^{r}-1\right)=\left(t^{s i}-1\right)$, and dividing Equation (3) by the equality $\left(1+x^{2} r_{i}^{-2}\right)=\left(1+x^{2} s_{j}^{-2}\right)$ we obtain corresponding equalities with $n$ replaced by $n-1$. (The reader should verify that both divisions are legitimate.) Hence the desired conclusion follows from the induction hypothesis.

Case 2. Suppose that $r_{1}=r_{2}, r_{3}=r_{4}, \cdots, r_{n-1}=r_{n}$ and that $s_{1}=s_{2}$, $\cdots, s_{n-1}=s_{n}$, where $n=2 k$. It will be convenient to introduce the abbreviations

so that

$$
a_{i} \equiv \stackrel{-2}{r_{2 i}}, \quad b_{j} \equiv \overrightarrow{s_{2 j}^{2}}(\bmod m),
$$

$$
\left(1+a_{1} x^{2}\right)^{2} \cdots\left(1+a_{k} x^{2}\right)^{2}=\left(1+b_{1} x^{2}\right)^{2} \cdots\left(1+b_{k} x^{2}\right)^{2} .
$$

Since $m$ is odd, note that every mixed cohomology class of the form

$$
1+c_{1} x+c_{2} x^{2}+\cdots \in \sum H^{2 i}(L ; Z)
$$

has a unique square root $1+d_{1} x+d_{2} x^{2}+\cdots$ of the same form. That is the congruences

$$
c_{1} \equiv 2 d_{1}, \quad c_{2} \equiv 2 d_{2}+d_{1}^{2}, \quad c_{3} \equiv 2 d_{3}+2 d_{1} d_{2}, \cdots
$$

have a unique solution. Therefore we can take the square root of Equation $\left(3^{\prime}\right)$ to obtain

$$
\left(1+a_{1} x^{2}\right) \cdots\left(1+a_{k} x^{2}\right)=\left(1+b_{1} x^{2}\right) \cdots\left(1+b_{k} x^{2}\right) .
$$

Since $x^{2 i} \neq 0$ for $i<k$ this proves that the ith elementary symmetric function of $a_{1}, \cdots, a_{k}$ is congruent to the ith elementary symmetric function of $b_{1}, \cdots, b_{k}$ for $i<k$. But the congruence

$$
a_{1} \cdots a_{k} \equiv b_{1} \cdots b_{k} \quad(\bmod m)
$$

for the $k$ th elementary symmetric function is also valid, by Lemma 12.19.

Consider the polynomials

$$
\left(y-a_{1}\right)\left(y-a_{2}\right) \cdots\left(y-a_{k}\right) \text { and }\left(y-b_{1}\right)\left(y-b_{2}\right) \cdots\left(y-b_{k}\right)
$$

in one variable over the field $\boldsymbol{Z}_{m}$. Since the corresponding elementary symmetric functions are all equal, we have

$$
\left(y-a_{1}\right)\left(y-a_{2}\right) \cdots\left(y-a_{k}\right)=\left(y-b_{1}\right)\left(y-b_{2}\right) \cdots\left(y-b_{k}\right) .
$$

According to the unique factorization theorem for polynomials over $Z_{m}$ this is only possible if $\left\{a_{1}, \cdots, a_{k}\right\}$ is a permutation of 
$\left\{b_{1}, \cdots, b_{k}\right\}$. But if, say, $a_{1} \equiv b_{j}(\bmod m)$ then $r_{2}^{2} \equiv s_{2 j}^{2}$, hence $r_{2} \equiv \pm s_{2 j}$, hence $r_{2}=s_{2 j}$ (making use of (2)).

Thus Case 2 has been reduced to Case 1 . This completes the proof of Lemma 12.20, and therefore completes the proof of the Theorem 12.15 .

Appendix 1. The congruence subgroup theorem. Let $A$ be a commutative ring, and $\mathfrak{a} \subset A$ an ideal such that the quotient ring $A / \mathfrak{a}$ is finite. The kernel of the natural homomorphism

$$
\mathrm{SL}(n, A) \rightarrow \mathrm{SL}(n, A / \mathfrak{a})
$$

will be denoted by $N_{\mathfrak{a}}$, and called the congruence subgroup corresponding to $\mathfrak{a}$. Clearly $N_{\mathfrak{a}}$ is a normal subgroup of finite index in $\mathrm{SL}(n, A)$.

Definition. The group $\mathrm{SL}(n, A)$ satisfies the congruence subgroup theorem if every subgroup of finite index in $\operatorname{SL}(n, A)$ contains such a congruence subgroup.

This theorem is true for $\mathrm{SL}(n, \boldsymbol{Z})$ providing that $n \geqq 3$. (See Bass, Lazard and Serre [1964]; or Mennicke [1965].) It is not true however for $\operatorname{SL}(2, \boldsymbol{Z})$. (See for example Reiner [1958].)

Now let $\theta$ be the ring of integers in an algebraic number field.

Assertion. If $n$ is sufficiently large, then $\operatorname{SL}(n, \mathcal{\theta})$ satisfies the congruence subgroup theorem.*

This result has been claimed by Mennicke [1965, p. 37]; and by Bass (unpublished). Assuming that it is true, we will prove the following.

THEOREM 1.6. If $\Pi$ is a finite abelian group, then $S K_{1}(Z \Pi)=0$.

The proof (due to Bass) will be based on five lemmas.

Lemma 1. If $\mathrm{SL}\left(n, A_{1}\right)$ and $\mathrm{SL}\left(n, A_{2}\right)$ both satisfy the congruence subgroup theorem, then so does $\mathrm{SL}\left(n, A_{1} \times A_{2}\right)$.

PROoF. This follows easily from the natural isomorphism

$$
\mathrm{SL}\left(n, A_{1} \times A_{2}\right)=\mathrm{SL}\left(n, A_{1}\right) \times \mathrm{SL}\left(n, A_{2}\right) .
$$

Consider next a ring $A$ and a subring $A_{0} \subset A$. Suppose that the

* Added in proof. At this date (April, 1966) it is not at all sure that this assertion is true for all number fields. 
additive group of $A$ is finitely generated, and that $A_{0}$ is (additively) a subgroup of finite index, say $\lambda$, in $A$.

Lemma 2. If $\mathrm{SL}(n, A)$ satisfies the congruence subgroup theorem, then so does $\mathrm{SL}\left(n, A_{0}\right)$.

Proof. Note that $\operatorname{SL}\left(n, A_{0}\right)$ is a subgroup of finite index in $\mathrm{SL}(n, A)$. In fact $\mathrm{SL}\left(n, A_{0}\right)$ clearly contains the congruence subgroup which corresponds to the ideal $\lambda A \subset A$. The rest of the argument is now quite easy.

Let $I I$ be a finite abelian group with $k$ elements.

LEMma 3. If $n$ is sufficiently large, then $\mathrm{SL}(n, Z \mathrm{ZI})$ satisfies the congruence subgroup theorem.

Proof. The semisimple algebra $Q \Pi I$ is isomorphic to a cartesian product of fields:

$$
Q I I \cong F_{1} \times \cdots \times F_{q} .
$$

Let $\Theta_{i}$ be the ring of integers in the number field $F_{i}$. Then clearly $Z \Pi$ is a subring of the product $\theta_{1} \times \cdots \times \theta_{q}$. The additive group of this product ring is clearly $\boldsymbol{Z}$-free of rank $k$. Similarly the additive group of $Z \Pi$ is free of rank $k$.

Choose $n$ so large that each of the groups $\operatorname{SL}\left(n, \mathcal{O}_{i}\right)$ satisfies the congruence subgroup theorem. Then Lemmas 1 and 2 imply that $\mathrm{SL}(n, Z \Pi)$ must also satisfy the congruence subgroup theorem. This proves Lemma 3.

Finally we will need the following.

Lemma 4. The group SL $(n, Z \Pi)$ is finitely generated.

(Compare Siegel [1943], Borel and Harish-Chandra [1962, §6.12].) This is perhaps best proved by noting that $\operatorname{SL}(n, Z \Pi)$ is a subgroup of finite index in

$$
\operatorname{SL}\left(n, \mathcal{O}_{1}\right) \times \cdots \times \operatorname{SL}\left(n, \mathcal{O}_{q}\right) .
$$

Since each $\operatorname{SL}\left(n, \mathcal{O}_{i}\right)$ is finitely generated by a theorem of Hurwitz [1895], the conclusion follows.

Lemma 5. If $n$ is sufficiently large, then the group $\mathrm{SL}(n, z \Pi)$ is equal to its own commutator subgroup.

Proof. Otherwise SL $(n, Z \mathrm{II})$ could be mapped homomorphically onto some finite, nontrivial abelian group $G$, making use of Lemma 4. The kernel of this homomorphism would contain a congruence subgroup $N_{\mathfrak{a}}$, by Lemma 3 . Hence the quotient 


$$
\mathrm{SL}(n, Z \Pi) / N_{\mathfrak{a}}
$$

would also map homomorphically onto $G$.

Recall that $N_{\mathfrak{a}}$ is defined by the exact sequence

$$
0 \rightarrow N_{\mathfrak{a}} \rightarrow \mathrm{SL}(n, Z \mathrm{ZI}) \stackrel{j}{\rightarrow} \mathrm{SL}(n, Z \mathrm{II} / \mathfrak{a}) .
$$

But $Z \Pi / \mathfrak{a}$ is a finite ring; and so has only finitely many maximal ideals. An easy argument then shows that the group $\operatorname{SL}(n, Z \Pi / \mathfrak{a})$ is generated by elementary matrices. (Compare Bass, Lazard, Serre [1964, Lemme 1], as well as $\$ 1.4$.) Hence the homomorphism $j$ must be onto. Furthermore the group

$$
\mathrm{SL}(n, Z \Pi) / N_{\mathfrak{a}} \cong \mathrm{SL}(n, Z \Pi / \mathfrak{a})
$$

must be equal to its own commutator subgroup (assuming that $n \geqq 3$ ). It follows that this group cannot map homomorphically onto the abelian group $G$. This contradiction completes the proof of Lemma 5 .

Since Theorem 1.6 clearly follows from Lemma 5, this completes the argument.

Appendix 2. The group $K_{0} A$ of virtual projective $A$-modules. Closely related to $K_{1}$ is the functor $K_{0}$ which is defined as follows. For each ring $A$ let $K_{0} A$ be the additive group having one generator $(P)$ for each finitely generated projective module $P$ over $A$, and one relation

$$
(P \oplus Q)=(P)+(Q)
$$

for each pair of finitely generated projectives. ${ }^{13}$ Note that any ring homomorphism $h: A \rightarrow A^{\prime}$ gives rise to a group homomorphism

$$
h_{*}: K_{0} A \rightarrow K_{0} A^{\prime}
$$

where

$$
h_{*}(P)=\left(A_{A}^{\prime} \underset{A}{\otimes} P\right) .
$$

Thus $K_{0}$ is a covariant functor from rings to additive groups.

(If $A$ happens to be commutative then the product operation

$$
(P)(Q)=(P \underset{A}{\otimes} Q)
$$

makes $K_{0} A$ into a ring. Thus $K_{0}$ can also be considered as a functor from commutative rings to commutative rings.)

The class of the free $A$-module of rank 1 generates a cyclic subgroup of $K_{0} A$. The quotient

${ }^{13}$ In other words $K_{0} A$ is the "Grothendieck group" associated with the category of finitely generated projectives over $A$. 
$K_{0} A /$ (subgroup generated by free modules)

is called the projective class group $\tilde{K}_{0} A$. (Serre [1958], Rim [1961].) Clearly $\tilde{K}_{0}$ is also a covariant functor. Here are some examples.

Example 1. If $A$ is a simple algebra then $K_{0} A$ is infinite cyclic, and $\widetilde{K}_{0} A$ is finite cyclic.

Example 2. If $A$ is a Dedekind ring then $\tilde{K}_{0} A$ can be identified with the ideal class group of $A$. (See Kaplansky [1952], Rim [1959].) The ring $K_{0} A$ splits additively as a direct sum

$$
K_{0} A \cong \boldsymbol{Z} \oplus \tilde{K}_{0} A,
$$

where $\tilde{K}_{0} A$ is an ideal, and where the product of any two elements in $\widetilde{K}_{0} A$ is zero. More generally:

ExAMPLE 3. If $A$ is any ring which admits a homomorphism into a skew-field $F$ then the homomorphisms

$$
Z \rightarrow A \rightarrow F
$$

give rise to a direct sum decomposition

$$
K_{0} A \cong Z \oplus \tilde{K}_{0} A \text {. }
$$

EXAMPLE 4. Let $T_{p}$ be the cyclic group of prime order $p$, and let $\xi=\exp (2 \pi i / p)$. Then according to Rim [1959] the homomorphism

$$
Z T_{p} \rightarrow Z[\xi]
$$

give rise to an isomorphism

$$
\tilde{K}_{0} Z T_{p} \rightarrow \tilde{K}_{0} Z[\xi] .
$$

Thus $\tilde{K}_{0} Z T_{p}$ is a finite group, with order equal to the class number of the cyclotomic field. These groups are trivial for $p<23$; however ${ }^{14}$

$$
\begin{aligned}
& \tilde{K}_{0} Z T_{23} \cong Z_{3} \oplus(?), \\
& \tilde{K}_{0} Z T_{29} \cong Z_{2} \oplus Z_{2} \oplus Z_{2} \oplus(?), \\
& \tilde{K}_{0} Z T_{31} \cong Z_{9} \oplus(?), \\
& \tilde{K}_{0} Z T_{37} \cong Z_{37} \oplus(?),
\end{aligned}
$$

and so on. These groups are surely nontrivial for all $p \geqq 23$.

More generally consider the group ring $Z \mathbb{Z I I}$ of an arbitrary multiplicative group.

EXAMPLE 5 (Swan [1960]). If $I I$ is finite, then the projective class group $\widetilde{K}_{0}(Z I I)$ is also finite.

EXAmple 6 (BAss, Heller and Swan [1964]). If $\Pi$ is free abelian, then $\tilde{K}_{0}(Z I I)=0$.

${ }^{14}$ See Kummer [1851] and [1860], as well as \$12.18. Here (?) denotes a finite group which is probably zero. 
In contrast, the following result has recently been obtained.

Example 7 (M. P. Murthy; UnPublished). If $\Pi=T \times T_{p}{ }^{2}$ is the product of a free cyclic group and a cyclic group of order $p^{2}$, then $\widetilde{K}_{0}(Z \Pi)$ contains infinitely many distinct elements of order $p$. In particular, this projective class group is not finitely generated.

To confuse matters, both $K_{0}$ and $K_{1}$ sometimes behave as covariant functors. Let

$$
h: A \rightarrow A^{\prime}
$$

be a ring homomorphism with the special property that $A^{\prime}$ is finitely generated and projective when considered as a left $A$-module. Then every finitely generated projective module $P$ over $A^{\prime}$ can be thought of as a finitely generated projective module $h^{*} P$ over $A$. Thus one obtains a backwards homomorphism

$$
h^{*}: K_{0} A^{\prime} \rightarrow K_{0} A
$$

which might be called the "transfer." Similarly a "transfer" homomorphism

can be defined. ${ }^{15}$

$$
h^{*}: K_{1} A^{\prime} \rightarrow K_{1} A
$$

The precise affinity between $K_{0}$ and $K_{1}$ is a little difficult to explain. (See Bass [1964], as well as the thesis of Gersten [1965].) One relationship can be described as follows: Given rings $A \subset A^{\prime}$ there exists an exact sequence of the form

$$
K_{1} A \rightarrow K_{1} A^{\prime} \rightarrow * \rightarrow K_{0} A \rightarrow K_{0} A^{\prime} .
$$

Here is a simple example. Let $A$ be a Dedekind ring and $F$ its quotient field. Then there is an exact sequence

$$
K_{1} A \rightarrow K_{1} F \rightarrow G \rightarrow K_{0} A \rightarrow K_{0} F \rightarrow 0,
$$

where $G$ denotes the group of all fractional $A$-ideals in $F$.

For any commutative $A$ it is possible to define ${ }^{16}$ a product operation

$$
K_{0} A \otimes K_{1} A \rightarrow K_{1} A,
$$

making $K_{1} A$ into a module over the ring $K_{0} A$.

${ }^{15}$ Represent an arbitrary element of $K_{1} A^{\prime}$ by an automorphism $\alpha$ of the free module $A^{\prime} \oplus \cdots \oplus A^{\prime}$. Choose a projective $A$-module $Q$ so that $h^{*}\left(A^{\prime} \oplus \cdots \oplus A^{\prime}\right) \oplus Q$ is $A$-free. Now the automorphism $\alpha \otimes$ (identity) of this free module determines the required element of $K_{1} A$.

${ }_{16}$ The product of an element $(P) \in K_{0} A$ and an element $(\alpha)$ of $K_{1} A$, where $\alpha$ denotes an automorphism of an $A$-free module $M$, is represented by the automorphism (identity $P$ ) $\otimes \alpha$ of the projective module $P \otimes M$. (Compare the preceding footnote.) 
Here is another example of a relation between $K_{0}$ and $K_{1}$. Let $T$ be an infinite cyclic group and $\Pi$ an arbitrary group. Then according to Bass, Heller and Swan $[1964, \S 5]$ there is a direct sum decomposition

$$
K_{1}(Z(T \times \Pi)) \cong K_{1}(Z \Pi) \oplus K_{0}(Z \Pi) \oplus(?),
$$

where (?) denotes an unknown group. Hence

$$
\mathrm{Wh}(T \times \Pi) \cong \mathrm{Wh}(\Pi) \oplus \tilde{K}_{0}(Z \Pi) \oplus(?) .
$$

If $\Pi$ is finite abelian of order $m$, then Bass has recently shown that the unknown third summand is an $m$-primary group.

As an example suppose that $\Pi$ is the cyclic group $T_{23}$ of order 23 . Then $\mathrm{Wh}(\mathrm{II})$ is free abelian of rank 10. The projective class group $\widetilde{K}_{0}\left(\boldsymbol{Z} T_{23}\right)$ is isomorphic to the ideal class group of $\boldsymbol{Q}[\exp (2 \pi i / 23)]$ and hence (presumably) is cyclic of order 3. (Example 4 above.) Thus

$$
\mathrm{Wh}\left(Z\left(T \times T_{23}\right)\right) \cong 10 Z \oplus Z_{3} \oplus \text { (?). }
$$

The conjugation operation can be explicitly computed in this case. It turns out to be multiplication by -1 on the $Z_{3}$ summand, and multiplication by +1 on the $\boldsymbol{Z} \oplus \cdots \oplus \boldsymbol{Z}$ summand.

As a second example let $\Pi=T \times T_{p^{2}}$ where $p$ is prime. Using $E x$ ample 7 above (M. P. Murthy) we see that the Whitehead group

$$
\mathrm{Wh}(T \times \Pi)=\mathrm{Wh}\left(T \times T \times T_{p^{2}}\right)
$$

is not finitely generated.

The geometric applications of the functor $K_{1}$ are strikingly paralleled in Wall's theory of obstructions to finiteness for CW-complexes. Let $X$ be a topological space which is dominated by a finite CWcomplex. Then Wall defines an obstruction

$$
\sigma \in \tilde{K}_{0}\left(Z_{\pi_{1}}(X)\right)
$$

which vanishes if and only if $X$ has the homotopy type of a finite CW-complex. (Wall [1965]. See also Siebenmann [1965].) This obstruction can be considered as a generalization of the familiar Euler characteristic.

Here is a quite different example from topology. Let $C^{\boldsymbol{X}}$ denote the ring of continuous complex valued functions on a compact space $X$. Then every finitely generated projective module over $\boldsymbol{C}^{\boldsymbol{X}}$ is isomorphic to the module of sections of a unique complex vector bundle over $X$. (Swan [1962].) Hence $K_{0} C^{X}$ can be identified with the 
Atiyah-Hirzebruch group $K^{0} X$ of virtual complex vector bundles over $X$. (See Atiyah and Hirzebruch [1959], Adams [1962].)

Similarly the special Whitehead group $S K_{1}\left(C^{X}\right)$ is isomorphic to the group of all homotopy classes of mappings from $X$ to the infinite special linear group SL(C). This group of homotopy classes is intimately related to the Atiyah-Hirzebruch group $K^{-1} X$.

Similarly the functors $K_{0}$ and $K_{1}$ applied to the ring $R^{X}$ of continuous real valued functions on $X$ closely related to the AtiyahHirzebruch groups $K O^{\circ}(X)$ and $K O^{-1}(X)$. Consider for example a space $X$ and a matrix

$$
\left(a_{i j}\right) \in \mathrm{SL}\left(n, R^{X}\right)
$$

Then the correspondence

$$
x \mapsto\left(a_{i j}(x)\right)
$$

maps $X$ into $\operatorname{SL}(n, R)$. Clearly any elementary row operation on the matrix induces a homotopy of the corresponding mapping.

ExAmple. Let $X$ be the unit circle in the plane, and let

$$
x, y \in R^{x}
$$

denote the two coordinate mappings. Then the matrix

$$
\left(\begin{array}{rr}
x & y \\
-y & x
\end{array}\right) \in \mathrm{SL}\left(2, R^{x}\right) \subset \mathrm{SL}\left(R^{x}\right)
$$

determines a mapping

$$
X \rightarrow \mathrm{SL}(2, R) \subset \mathrm{SL}(R)
$$

which clearly represents a generator for the group $\pi_{1} \mathrm{SL}(R)$. (Recall that $\pi_{1} \mathrm{SL}(R) \cong \pi_{1} O$ is cyclic of order 2.) It follows that the matrix

$$
\left(\begin{array}{rr}
x & y \\
-y & x
\end{array}\right)
$$

over $R^{X}$ can not be expressed as a product of elementary matrices. This conclusion follows a fortiori if we restrict attention to the subring

(Compare §1.7.)

$$
R[x, y] \subset R^{x} .
$$

\section{REFERENCES}

1851

E. E. KUMMER

Sur la thêrie des nombres complexes composés de racines de l'unitê et de nombres entiers, J. Math. (Liouville) 16, 377-498. 
1860

E. E. Kummer

1870

E. E. KuMmER

1874

E. E. KUMMER

1875

G. G. REUSChLE

1886

P. WOLFSKEHL

1891

H. MinkowsKI

1895

A. HuRwitz

1928

H. S. VANDIVER and

G. E. WAHLIN

1935

W. Franz

K. REIDEMEISTER

1938

M. RUEFF

1939

G. DE RHAM

J. H. C. WhitehEAD 1940

G. HigmaN

J. H. C. WhiteheAD 1941

J. H. C. WHITEHEAD
Bemerkungen $z u$ den aus $29^{\text {ten }}$ Einheitswurzeln gebildeten Zahlen, Monatsber. K. Akad. Wiss. Berlin, 734-735.

Über eine Eigenschaft der Einheiten der aus den Wurzeln der Gleichung $\alpha^{\lambda}=1$ gebildeten complexen Zahlen und über den zweiten Faktor der Klassenzahl, Monatsber. K. Akad. Wiss. Berlin, 855-880.

Über diejenigen Primzahlen $\lambda$, filr welche die Klassenzahl der aud $\lambda$-ten Einheitswurzeln gebildeten complexen Zahlen durch $\lambda$ theilbar ist, Monatsber. K. Akad. Wiss. Berlin, 239-248.

Tafeln complexer Primzahlen welche aus Wurseln der Einheit gebildet sind, Berlin K. Akad. Wiss.

Beweis dass der zweite Faktor der Klassenanzahl für die aus den elften und dreizehnten Einheitswurzeln gebildeten Zahlen gleich Eins ist, J. Riene Angew. Math. 99, 173-178.

Ueber die positiven quadratischen Formen und über kettenbruchähnliche Algorithmen, J. Reine Angew. Math. 107, 278297.

Die unimodularen Substitutionen in einem algebraischen Zahlenkörper, Mathematische Werke 2, 244-268.

Algebraic numbers. II, Bull. Nat. Res. Council, 62.

Über die Torsion einer Überdeckung, J. Reine Angew. Math. 173, 245-254.

Homotopieringe und Linsenräume, Hamburger Abhandl. 11, 102-109.

Beiträge sur Untersuchung der Abbildungen von Mannigfaltigkeiten, Compositio Math. 6, 161-202.

Sur les complexes avec automorphismes, Comment. Math. Helv. 12, 191-211.

Simplicial spaces, nuclei and m-groups, Proc. London Math. Soc. 45, 243-327.

The units of group rings, Proc. London Math. Soc. 46, 231248.

On $C^{1}$-complexes, Ann. of Math. 41, 809-824.

On incidence matrices, nuclei and homotopy types, Ann. of Math. 42, 1197-1239. 
1943

J. Dieudonne

C. L. Siegel 1949

J. H. C. WHITEHEAD

1950

K. REIDEMEISTER

G. DE RHAM

J. H. C. WhiteheAD 1952

H. HASSE

I. KAPLANSKY

1953

P. OluM

E. Artin 1957

1958

I. REINER

J.-P. Serre

1959

M. Ativah and

F. HIRZEBRUCH

D. S. RIM

1960

R. G. SwaN

1961

J. MILNOR

D. S. RIM

S. Smale

A. H. WALIACE

1962

J. F. ADAMS

H. BASS and

S. Schanuel
Les determinants sur un corps non commutatif, Bull. Soc. Math. France 71, 27-45.

Discontinuous groups, Ann. of Math. 44, 674-689.

Combinatorial homotopy. I, Bull. Amer. Math. Soc. 55, 213245.

Complexes and homotopy chains, Bull. Amer. Math. Soc. 56, 297-307.

Complexes a automorphismes et homeomorphie différentiables, Ann. Inst. Fourier, Grenoble 2, 51-67.

Simple homotopy types, Amer. J, Math. 72, 1-57.

Über die Klassenzahl abelscher Zahlkörper, Akademie-Verlag, Berlin.

Modules over Dedekind rings and valuation rings, Trans. Amer. Math. Soc. 72, 327-340.

Mappings of manifolds and the notion of degree, Ann. of Math. 58, 458-480.

Geometric algebra, Interscience, New York.

Normal subgroups of the unimodular group, Illinois J. Math. 2, 142-144.

Modules projectifs et espaces fibrés a fibre vectorielle, Séminaire Dubreil, Algèbre et theorie des nombres, Paris.

Riemann-Roch theorems for differentiable manifolds, Bull. Amer. Math. Soc. 65, 276-281.

Modules over finite groups, Ann. of Math. 69, 700-712.

Induced representations and projective modules, Ann. of Math. 71, 552-578.

Two complexes which are homeomorphic but combinatorially distinct, Ann. of Math. 74, 575-590.

On projective class groups, Trans. Amer. Math. Soc. 98, 459467.

Generalized Poincare's conjecture in dimensions greater than four, Ann. of Math. 74, 391-406.

Modifications and cobounding manifolds. II, J. Math. Mech. 10, 773-809.

Vector fields on spheres, Ann. of Math. 75, 603-632.

The homotopy theory of projective modules, Bull. Amer. Math. Soc. $68,425-428$. 
A. BoREL and HARISH-ChANDRA

J. MILNOR

S. Smale

R. G. SWAN

R. G. SwaN

1963

D. BARDEN

B. MAZUR

J. MILNoR

J. MunkRes

1964

H. BASS

H. BASS

H. Bass, A. Heller, and R. SwaN

H. BASS, M. LAZARD, and J.-P. SERRE

J. MiLnor

G. SchrutKa v.

RECHTENSTAMM

G. DE RHAM

G. DE RHAM

J. Stallings

R. Szczarba

C. T. C. WALL

1965

J. FolKMAN

S. Gersten
Arithmetic subgroups of aigebraic groups, Ann. of Math. 75, 485-535.

A duality theorem for Reidemeister torsion, Ann. of Math. 76, 137-147.

On the structure of manifolds, Amer. J. Math. 84, 387-399.

Vector bundles and projective modules, Trans. Amer. Math. Soc. 105, 264-277.

Projective modules over group rings and maximal orders, Ann. of Math. 76, 55-61.

The structure of manifolds, $\mathrm{Ph} . \mathrm{D}$. Thesis, Cambridge University, Cambridge, England

Relative neighborhoods and the theorems of Smale, Ann. of Math. 77, 232-249.

Morse theory, Princeton Univ. Press, Princeton, N. J.

Elementary differential topology, Princeton Univ. Press, Princeton, N. J.

The stable structure of quite general linear groups, Bull. Amer. Math. Soc. 70, 429-433.

$K$-theory and stable algebra, Publ. de l'Inst. des Hautes Etudes Sci. \#22.

The Whitehead group of a polynomial extension, Publ. de l'Inst. des Hautes Etudes Sci. \#22.

Sous-groupes d'indice fini dans $\mathrm{SL}(n, Z)$, Bull. Amer. Math. Soc. 70, 385-392.

Some free actions of cyclic groups on spheres, Differential Analysis, Tata Institute and Oxford University Press, 37-42. Tabelle der (Relativ)-Klassenzahlen der Kreiskörper . . . , Abh. Deutsch. Akad. Wiss. Berlin, \#2, 64 pp.

(1), Reidemeister's torsion invariant and rotations of $S^{n}$, Differential Analysis, Tata Institute and Oxford Univ. Press, 27-36.

(2), Torsion et type simple d'homotopy, Séminaire Math. Université de Lausanne. (Lectures by Kervaire, Maumary, and de Rham.)

Projective class groups and Whitehead groups, (mimeographed) Rice University, Houston, Texas.

On tangent bundles of fibre spaces and quotient spaces, Amer. J. Math. 86, 685-697.

Differential topology, Part IV, Theory of handle decompositions (mimeographed), Cambridge University, Cambridge, England.

Equivariant maps of spheres into the classical groups, $\mathrm{Ph} . \mathrm{D}$. Thesis, Princeton University, Princeton, N. J.

(1), Whitehead groups of free associative algebras, Bull. Amer. Math. Soc. 71, 157-159. 
S. Gersten

K. W. KWUN and

R. H. SzCZARBA

J. L. MeNNiCKe

J. MiLnoR

L. SiebenmanN

J. Sondow

J. Stallings

J. Stallings

C. T. C. WALL

1966

M. F. Atiyah and R. Botr

TO APPEAR:

H. BASS

H. BAss and M. P. MURThy
(2), Ph. D. Thesis, Cambridge University, Cambridge. Product and sum theorems for Whitehead torsion, Ann. of Math. 82, 183-190.

Finite factor groups of the unimodular group. Ann. of Math. 81, 31-37.

Lectures on the h-cobordism theorem, Notes by L. Siebenmann and J. Sondow, Princeton Univ. Press, Princeton, N. J.

The obstruction to finding a boundary for an open manifold of dimension greater than five, Ph. D. Thesis, Princeton University, Princeton, N. J.

Disproof of the Hauptvermutung for manifold pairs, $\mathrm{Ph} . \mathrm{D}$. Thesis, Princeton University, Princeton, N. J.

(1), Whitehead torsion of free products, Ann. of Math. 82, 354363.

(2), On infinite processes leading to differentiability in the complement of a point, Differential and Combinatorial Topology, (A Symposium in honor of M. Morse), Princeton University Press, Princeton, N. J., 245-254.

Finiteness conditions for CW-complexes, Ann. of Math. 81, 56-69.

A Lefschetz fixed point formula for elliptic differential operators, Bull. Amer. Math. Soc. 72, 245-250.

The Dirichlet unit theorem, induced characters, and Whitehead groups of finite groups.

Grothendieck groups and Picard groups of abelian group rings. 